University of Tennessee Health Science Center

UTHSC Digital Commons

7-2013

\title{
The Protumorigenic Role of Caspase-8 in Neuroblastoma
}

Devin Drew Twitchell

University of Tennessee Health Science Center

Follow this and additional works at: https://dc.uthsc.edu/dissertations

Part of the Amino Acids, Peptides, and Proteins Commons, Medical Cell Biology Commons, and the Neoplasms Commons

\section{Recommended Citation}

Twitchell, Devin Drew , "The Protumorigenic Role of Caspase-8 in Neuroblastoma" (2013). Theses and Dissertations (ETD). Paper 267. http://dx.doi.org/10.21007/etd.cghs.2013.0327.

This Dissertation is brought to you for free and open access by the College of Graduate Health Sciences at UTHSC Digital Commons. It has been accepted for inclusion in Theses and Dissertations (ETD) by an authorized administrator of UTHSC Digital Commons. For more information, please contact jwelch30@uthsc.edu. 


\title{
The Protumorigenic Role of Caspase-8 in Neuroblastoma
}

\begin{abstract}
Neuroblastoma (NB), the most common extracranial solid tumor in children, accounts for $15 \%$ of cancerrelated deaths in pediatric patients. Caspase-8 (casp8), a proapoptotic protein, is silenced in approximately, $50-70 \%$ of neuroblastoma patient samples. Loss of casp8 has been suggested to increase NB metastasis and correlated, in some studies, with advanced-stage NB. Furthermore, decreased casp8 expression may facilitate neuroblastoma tumorigenesis by protecting cells from cell death mediated by either integrins or chemotherapeutics. Paradoxically, casp8 expression is maintained in $30-50 \%$ of NB patient samples giving rise to the possibility that casp8 may provide selective advantages for NB tumorigenesis. Caspase-8 is shown to increase tumor cell adhesion, migration and growth. This drives the question as to how a single protein can promote very opposing functions. One possible explanation is that post- translational modifications may decrease the catalytic activity of casp8 and shift its role towards survival. Caspase-8 has been shown to be phosphorylated by SRC, thus decreasing its apoptotic function and increasing ERK signaling, which supports our hypothesis. Ataxia telangiectasia mutated kinase (ATM), an essential mediator in the DNA double strand break repair mechanism, is activated by chemotherapeutic treatments, radiation, and cellular stress. Caspase-8 has one consensus phosphorylation site that has been reported to be phosphorylated in a genome-wide screen for ATM substrates. Here, we tested whether casp8 expression enhanced primary NB tumorigenesis and whether modulation of casp8 by phosphorylation altered its biological function in NB. For the first time, this study demonstrates that casp8 enhances orthotopic xenograft tumor establishment in low tumorigenic neuroblastoma cells; which, may explain why casp8 expression is maintained in some NB tumors. Furthermore, exposure to DNA damaging agents suppresses the apoptotic function of casp8 via ATMmediated phosphorylation, thereby shifting the balance between the proapoptotic and prosurvival functions towards cell survival. This outlines a possible mechanism by which tumor cells may avoid cell death when exposed to chemotherapeutic agents.
\end{abstract}

\section{Document Type}

Dissertation

Degree Name

Doctor of Philosophy (PhD)

Program

Biomedical Sciences

Research Advisor

Gerard P. Zambetti, Ph.D.

\section{Keywords}

ATM, caspase-8, DNA damage, neuroblastoma

\section{Subject Categories}

Amino Acids, Peptides, and Proteins | Chemicals and Drugs | Diseases | Medical Cell Biology | Medical Sciences | Medicine and Health Sciences | Neoplasms 


\title{
THE PROTUMORIGENIC ROLE OF CASPASE-8 IN NEUROBLASTOMA
}

\author{
A Dissertation \\ Presented for \\ The Graduate Studies Council \\ The University of Tennessee \\ Health Science Center
}

\begin{abstract}
In Partial Fulfillment
Of the Requirements for the Degree

Doctor of Philosophy

From The University of Tennessee
\end{abstract}

By

Devin Drew Twitchell

August 2013 
Copyright (C) 2013 by Devin Drew Twitchell. All rights reserved. 


\section{DEDICATION}

This dissertation is dedicated to my wonderful parents who have given immeasurable love, devotion, guidance, and sacrifice throughout the years on my behalf and have helped to make this achievement possible.

A special dedication is extended to my lovely wife, who without her, none of this would have been possible. Through her unselfish love, devotion, and personal sacrifice for me and our family, she has been my strength, my support, and my motivation to endure when times got hard. To my wonderful children (Camryn, Leigha, Ethan, McKenna, and Hayden) who have kept me focused on what matters most in life. 


\section{ACKNOWLEDGEMENTS}

This work would not have been possible without the mentorship of Dr. Jill Lahti, who lost her battle to breast cancer. Dr. Jill Lahti was a great mentor who allowed a young scientist, such as me, to explore and develop my technique, logic, and scientific reasoning. I regret that she could not be here to see this work completed. I appreciate her willingness to accept me into her lab and work on her project as my own. She was always willing to listen to my ideas and theories, no matter how extravagant, and always encouraged me to explore many of those ideas.

Special thanks are extended to my current mentor Dr. Gerard Zambetti who was gracious enough to adopt me into his lab. I appreciate all the work he did behind the scenes to keep me at St. Jude long enough to complete my dissertation. Dr. Zambetti is an incredible mentor who is constantly urging me to challenge my hypothesis and myself as a researcher. He has given me great insight and understanding as to how be a good scientist, constantly questioning and challenging the hypothesis. Dr. Zambetti instilled in me a stronger desire to improve as a scientist each time we met.

I am also very thankful to my dissertation committee members, Dr. Andrew Davidoff, Dr. Peter McKinnon, Dr. Joseph Opferman, and Dr. Lawrence Pfeffer, for their good advice and direction.

Technical support has been essential in this work. In this regard, I would like to extend special appreciation to the Small Animal Imaging (SAI) team at St. Jude, especially Chris Calabrese, Melissa Johnson, and Shantel Brown for their expertise and work.

I am very grateful to all my friends, coworkers, and collaborators from both the Lahti and Zambetti labs. I thank Jose Grenet and Judy Hyle for all their technical support, assistance, and for making the lab work a fun play to work. I would like to thank Madoka Inoue for her constant work in supplying me with mice. I extend special thanks to all the previous lab members of the Lahti lab (Dr. Tal Teitz, Dr. Manrong Jiang, Dr. Dongli $\mathrm{Hu}$, and Dr. Akira Inoue) for their guidance, advice, and support. I extend special thanks to the Zambetti lab, which in my short time have made me feel accepted. I appreciate the help and counsel from Dr. Sean Garrison and for his help in experimental design. I also appreciate Emilia Pinto, Rob Jeffers, Jinling Wang, and Andre van Helvoot Lengert for their support and guidance. 


\begin{abstract}
Neuroblastoma (NB), the most common extracranial solid tumor in children, accounts for $15 \%$ of cancer-related deaths in pediatric patients. Caspase- 8 (casp8), a proapoptotic protein, is silenced in approximately, $50-70 \%$ of neuroblastoma patient samples. Loss of casp8 has been suggested to increase NB metastasis and correlated, in some studies, with advanced-stage NB. Furthermore, decreased casp8 expression may facilitate neuroblastoma tumorigenesis by protecting cells from cell death mediated by either integrins or chemotherapeutics. Paradoxically, casp8 expression is maintained in $30-50 \%$ of NB patient samples giving rise to the possibility that casp 8 may provide selective advantages for NB tumorigenesis. Caspase- 8 is shown to increase tumor cell adhesion, migration and growth. This drives the question as to how a single protein can promote very opposing functions. One possible explanation is that post-translational modifications may decrease the catalytic activity of casp8 and shift its role towards survival. Caspase- 8 has been shown to be phosphorylated by SRC, thus decreasing its apoptotic function and increasing ERK signaling, which supports our hypothesis. Ataxia telangiectasia mutated kinase (ATM), an essential mediator in the DNA double strand break repair mechanism, is activated by chemotherapeutic treatments, radiation, and cellular stress. Caspase- 8 has one consensus phosphorylation site that has been reported to be phosphorylated in a genome-wide screen for ATM substrates. Here, we tested whether casp8 expression enhanced primary NB tumorigenesis and whether modulation of casp 8 by phosphorylation altered its biological function in NB. For the first time, this study demonstrates that casp8 enhances orthotopic xenograft tumor establishment in low tumorigenic neuroblastoma cells; which, may explain why casp8 expression is maintained in some NB tumors. Furthermore, exposure to DNA damaging agents suppresses the apoptotic function of casp8 via ATM-mediated phosphorylation, thereby shifting the balance between the proapoptotic and prosurvival functions towards cell survival. This outlines a possible mechanism by which tumor cells may avoid cell death when exposed to chemotherapeutic agents.
\end{abstract}




\section{TABLE OF CONTENTS}

CHAPTER 1. INTRODUCTION .................................................................................

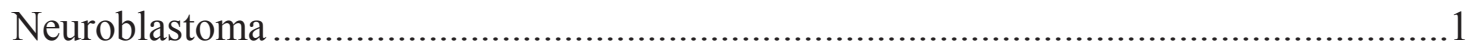

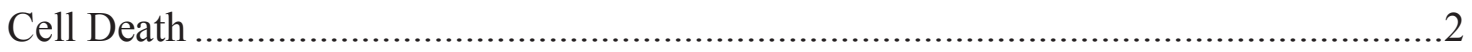

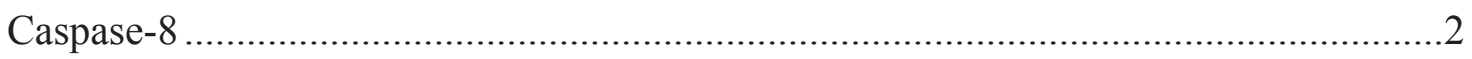

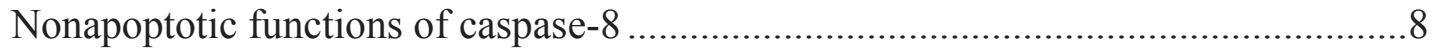

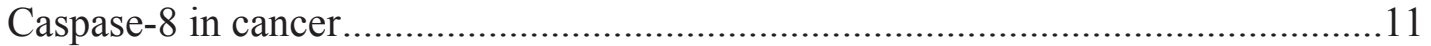

Role of caspase-8 in neuroblastoma.................................................................. 13

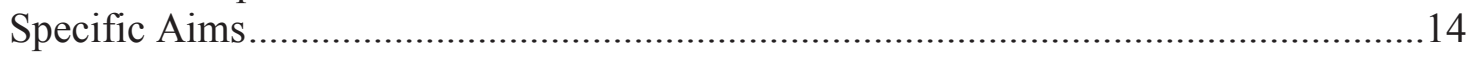

\section{CHAPTER 2. CASPASE-8 PROMOTES NEUROBLASTOMA}

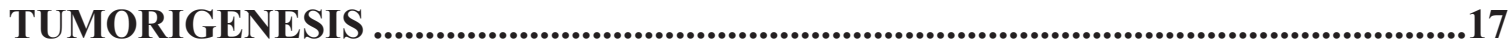

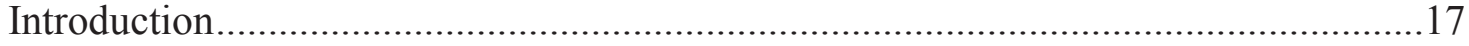

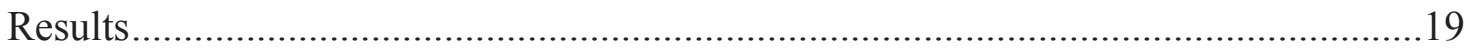

Caspase-8 expression promotes NB tumor growth in low tumorigenic cells ............19

Caspase-8 increased tumor engraftment and growth in NB7 cells .........................21

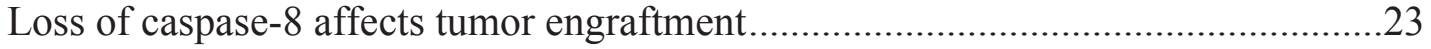

Tumorigenesis does not require the proteolytic activity of caspase -8 .....................24

Catalytically inactivated caspase- 8 did not enhance tumor engraftment ..................24

The protumorigenic function of caspase- 8 is dependent on the prodomain ..............26

Lack of the N-terminal prodomain suppresses tumor growth ...............................29

Overall expression of caspase- 8 is diminished in orthotopic xenografts ..................29

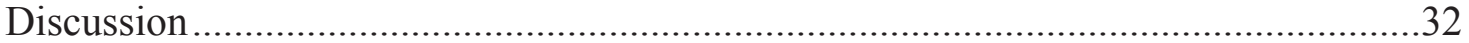

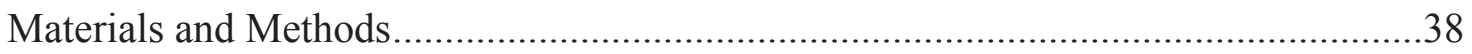

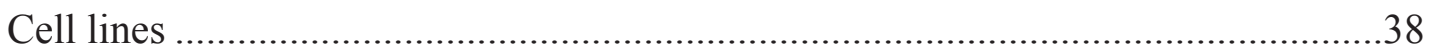

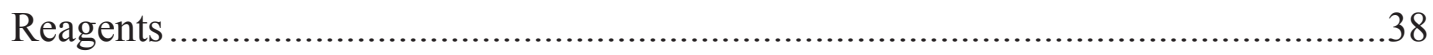

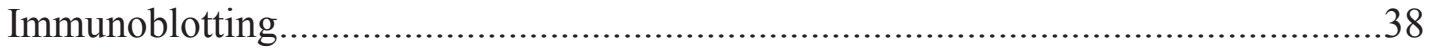

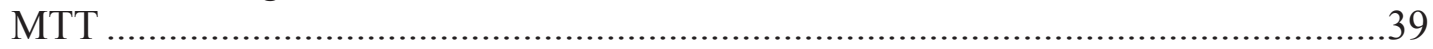

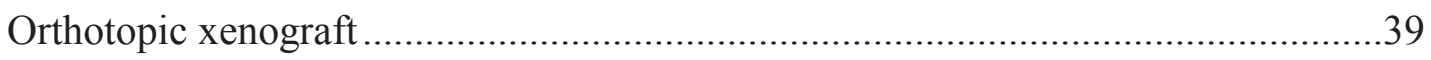

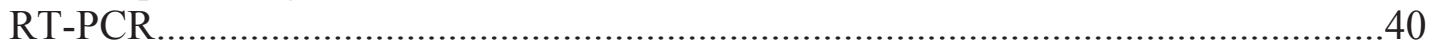

\section{CHAPTER 3. ROLE OF CASPASE-8 PHOSPHORYLATION IN}

NEUROBLASTOMA ........................................................................................................41

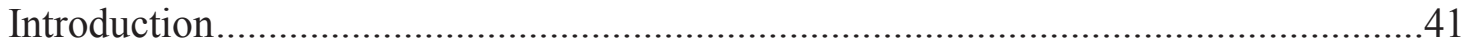

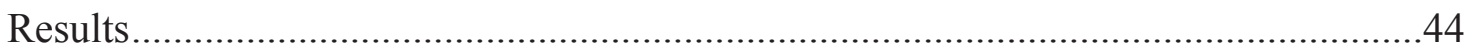

DNA damage promotes caspase-8 phosphorylation ..........................................44

ATM-mediated phosphorylation of caspase-8 …...................................................4

Caspase-8 phosphorylation is a cytoplasmic event ..............................................47

Serine 219 of caspase-8 suppresses the catalytic activity ...................................49

3D culture demonstrated increased colony formation ........................................49

Role of caspase- 8 phosphorylation in NB tumorigenesis .....................................52

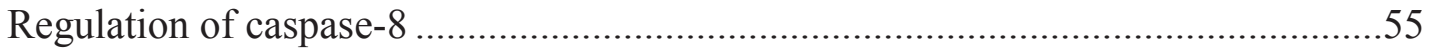

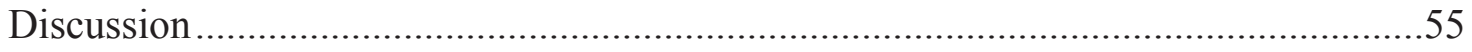

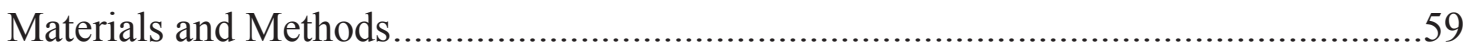




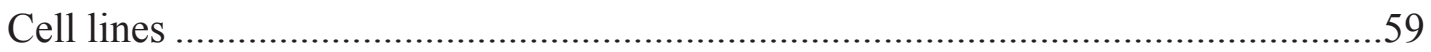

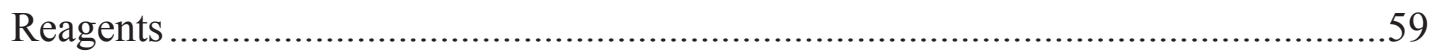

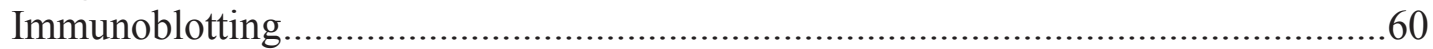

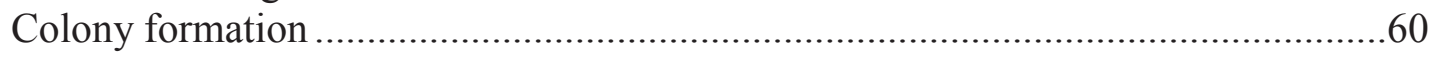

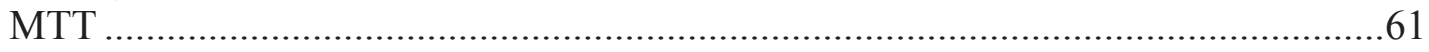

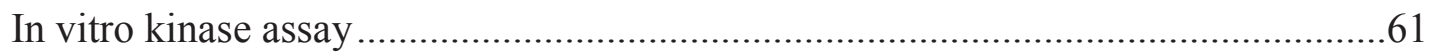

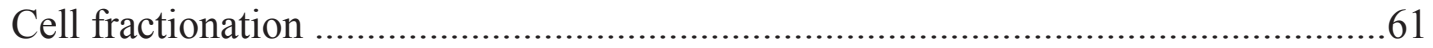

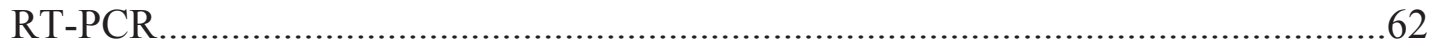

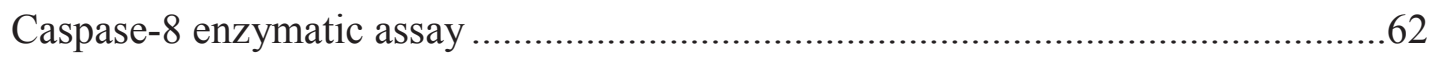

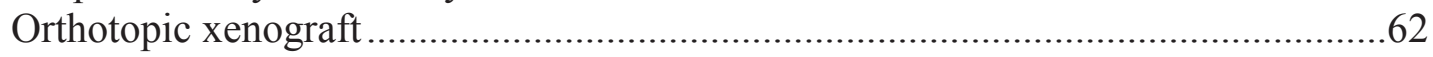

CHAPTER 4. OVERALL SUMMARY AND FUTURE EXPERIMENTS ...............64

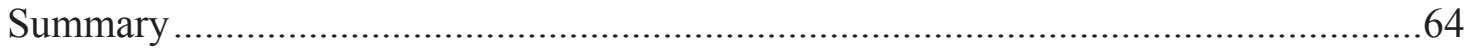

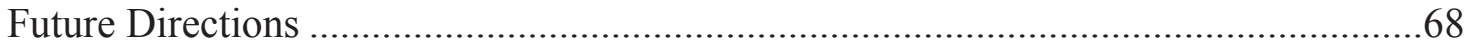

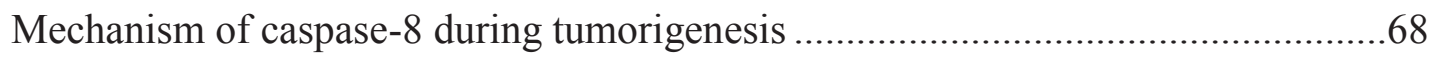

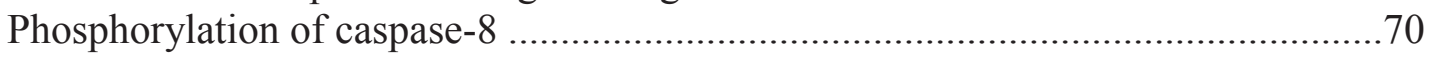

LIST OF REFERENCES ......................................................................................71

APPENDIX. CHAPTER 3 SUPPLEMENTAL FIGURES.........................................83

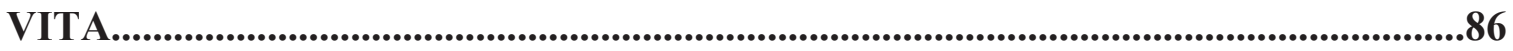




\section{LIST OF FIGURES}

Figure 1-1. Representation of the caspase-8 family and caspase-8 ..............................

Figure 1-2. Outline of the roles of caspase- 8 in various cell death pathways. ..................5

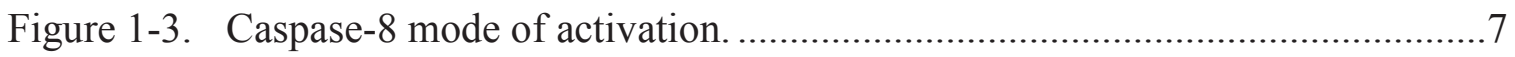

Figure 1-4. Prosurvival mechanisms of caspase- 8 . ...................................................

Figure 1-5. Domain specific functions of caspase-8. ........................................... 10

Figure 1-6. Role of caspase-8 expression levels promote tumorigenesis and

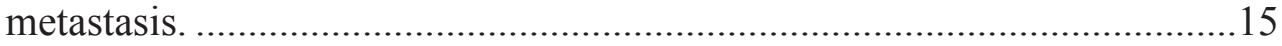

Figure 2-1. Caspase-8 increased neuroblastoma tumorgenicity. ...............................20

Figure 2-2. Increased engraftment in caspase-8 positive NB7 cells............................22

Figure 2-3. Catalytic activity of casp8 suppressed tumor growth. ..............................25

Figure 2-4. Inactivated of caspase-8 did not enhance tumor engraftment....................27

Figure 2-5. The prodomain is essential for caspase- 8 tumorgenicity..........................28

Figure 2-6. The N-terminal prodomain is required for tumor growth.........................30

Figure 2-7. Caspase-8 is diminished in orthotopic xenografts. ...................................

Figure 2-8. Overall expression of caspase-8 is diminished in orthotopic xenografts......33

Figure 3-1. Caspase-8 phosphorylation at S219 post DNA damage. ............................45

Figure 3-2. Caspase-8 phosphorylation at S219 is ATM dependent...........................46

Figure 3-3. Phosphorylation of caspase- 8 is a cytoplasmic event. ................................48

Figure 3-4. Phosphorylation at S219 suppresses caspase-8-mediated apoptosis. ...........50

Figure 3-5. Phosphorylation increased colony formation and growth. ..........................51

Figure 3-6. Phosphorylation of caspase-8 did not enhance tumorigenesis....................53

Figure 3-7. Phosphorylation did not affect tumor engraftment. ................................54

Figure 3-8. Posttranslational regulation of caspase-8 in S219A and S219E mutated caspase-8 expressing NB7 cells. ......................................................56 
Figure 4-1. Role of caspase-8 in neuroblastoma. ..................................................66

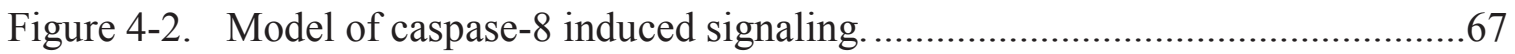

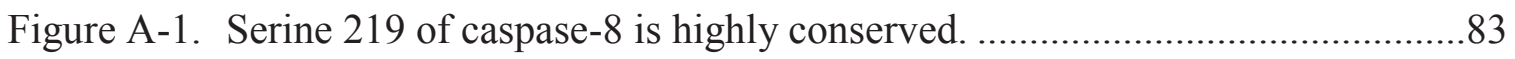

Figure A-2. Caspase- 8 phosphorylation in apoptosis, adhesion and tumor growth........84

Figure A-3. Caspase- 8 expression does not affect cell cycle. ...................................85 


\section{LIST OF ABBREVIATIONS}

$\begin{array}{ll}\gamma \text {-IFN } & \text { gamma interferon } \\ \text { ATG7 } & \text { autophagy-related gene 7 } \\ \text { ATM } & \text { ataxia telangiectasia mutated } \\ \text { BRCA1 } & \text { breast cancer 1, early onset } \\ \text { CARM } & \text { chick chorioallantoic membrane } \\ \text { CARMA 1 } & \text { caspase recruitment domain membrane-associated guanylate } \\ & \text { kinase protein 1 } \\ \text { Cdk1 } & \text { cyclin-dependent kinase 1 } \\ \text { Chk1 } & \text { checkpoint kinase 1 } \\ \text { Chk2 } & \text { checkpoint kinase 2 } \\ \text { cIAP } & \text { cellular inhibitor of apoptosis protein 1 } \\ \text { CUL3 } & \text { cullin3 } \\ \text { DD } & \text { death domain } \\ \text { DED } & \text { death effector domain } \\ \text { DISC } & \text { death-inducing signaling complex } \\ \text { DR4 } & \text { death receptor 4 } \\ \text { ECM } & \text { extracellular matrix } \\ \text { EGF } & \text { epidermal growth factor } \\ \text { FADD } & \text { fas-associated protein with death domains } \\ \text { FLIP } & \text { FLICE-inhibitory protein } \\ \text { G6PDH } & \text { glucose-6-phosphate dehydrogenase } \\ \text { HIF-1 } & \text { hypoxia-induced factor 1 } \\ \text { HNSCC } & \text { head and neck squamous cell carcinoma } \\ \text { HSP27 } & \text { heat-shock protein 27 } \\ \text { IKK } & \text { IkappaB kinase } \\ \text { IMD } & \text { integrin-mediated death } \\ \text { INSS } & \text { international neuroblastoma staging system } \\ \text { KAP-1 } & \text { KRAB-associated protein 1 } \\ \text { Malt 1 } & \text { mucosa-associated lymphoid tissue lymphoma translocation gene 1 } \\ \text { MKI } & \text { mitotic cells versus karyorrhexis } \\ \text { MOMP } & \text { mitochondrial outer membrane permeabilization } \\ \text { mTORC1 } & \text { mTOR complex 1 } \\ \text { NADPH } & \text { nicotinamide adenine dinucleotide phosphate-oxidase } \\ \text { NEMO } & \text { NF-kB essential modulator } \\ \text { NF-KB } & \text { nuclear factor kappa B } \\ \text { PARP2 } & \text { poly (adenosine diphosphate-ribose) polymerase 2 } \\ \text { Phox2b } & \text { paired-like homeobox 2B } \\ \text { PI3K } & \text { phosphatidylinositol 3-kinase } \\ \text { PIKK } & \text { phosphoinositide 3-kinase-related kinase } \\ \text { RIP1 } & \text { receptor-interacting protein 1 } \\ \text { RIP3 } & \text { receptor-interacting protein 3 } \\ \text { ROS } & \text { radical oxygen species } \\ \text { RSK2 } & \text { ribosomal S6 kinase 2 } \\ & \end{array}$


SCID

TNF

TNFR1

TRADD

TRAF $2 / 5$

TRAF6

TRAIL

TrkA

TrkB

TSC2

zVAD

4E-BP1

$53 \mathrm{BP} 1$ severe combined immunodeficiency

tumor necrosis factor

tumor necrosis factor receptor 1

tumor necrosis factor receptor-associated death domain

tumor necrosis factor associated factor $2 / 5$

tumor necrosis factor receptor associated factor 6

tumor necrosis factor-related apoptosis-inducing ligand

tropomyosin-receptor-kinase A

tropomyosin-receptor-kinase B

tuberous sclerosis complex 2

benzyloxycarbonylvalyl-alanyl-aspartic acid (O-methyl)-fluoromethylketone)

eIF-4E-binding protein 1

p53-binding protein 1 


\section{CHAPTER 1. INTRODUCTION}

\section{Neuroblastoma}

Neuroblastoma (NB) is the most common extracranial solid tumor diagnosed in children accounting for 7 to $10 \%$ of pediatric cancers and $15 \%$ of cancer-related deaths [1-4]. NB, considered to be a disease of the sympathetic nervous system, is derived from neural crest cells of the sympaticoadrenal lineage. Sympaticoadrenal cells give rise to the medullary region of the adrenal gland and the sympathetic ganglia $[5,6]$. NB may develop anywhere along the sympathetic axis including the neck $(5 \%)$, chest $(20 \%)$, pelvis $(5 \%)$, and abdominal cavity $(65 \%)$ of which the adrenal medulla is the most common site of tumorigenesis $[3,7,8]$.

The International Neuroblastoma Staging System (INSS) classifies NB into six distinct stages including stage 1, 2a, 2b, 3, 4 and 4s [3, 4, 8]. Early stage NB (stages 1, 2) are localized tumors clinically treated with irradiation and chemotherapy. Stages 3 and 4 are metastatic disease and are often resistant to chemotherapy. In contrast, $4 \mathrm{~s}$ tumors are metastatic yet regress spontaneously without clinical intervention [7,9]. Further classification of NB includes the level of Schwannian stromal content, age at which the patient is diagnosed, and the number of mitotic versus necrotic cells (karyorrhexis) [10]. Approximately, $75 \%$ of children presenting with advanced stage NB are older than 1 year of age $[3,11]$. Diagnosis and overall prognosis of NB is extremely variable based on age, tumor location, metastasis, and tumor heterogeneity. The overall five-year survival rate of NB patients has increased; however, patients diagnosed with advanced-stage NB have only shown slight improvements with less than a 40\% long-term survival rate despite advances in chemotherapeutic treatments over the last few decades [8].

Several genetic abnormalities have been associated with NB tumorigenesis. Familial NB only accounts for 5\% of NB deriving from germline mutations in the pairedlike homeobox 2B (PHOX2b) gene and a receptor tyrosine kinase known as ALK [12]. Amplification of the MYCN proto-oncogene occurs in approximately 33\% of NB patients. Chromosomal deletion or rearrangement of 1p36 (26\%), 2q33 (30\%), 11q23 (44\%), 14q23 (44\%) and gain of 17q23 (50\%) are also associated with NB [13]. Favorable prognosis is linked to near-triploidy status and expression of neurotrophin receptor known as tropomyosin-receptor-kinase A (TrkA). Poor prognosis may result from neurotrophin receptor TrkB expression, loss of $1 p, 11 p$, and gain of 17q [3, 14-17]. A hallmark of advanced-stage NB is the amplification of the 2p24 locus leading to high levels of N-Myc. N-Myc, a transcription factor, leads to dysfunctional cell growth when overexpressed. MYCN is amplified in 33\% of advanced-stage NB leading to a 3-year survival rate of only $30 \%[3,18]$. The exact mechanism of how MYCN amplification is associated with poor prognosis is still unclear. Research suggests that MYCN amplification may require deregulation of the apoptotic machinery in order to survive, since N-Myc sensitizes NB cells to death receptor-mediated apoptosis via casp8 activation [19]. Although it remains unclear as to which tumor suppressor genes reside in the above mentioned chromosomal regions, p16INK4a, pRb, p53, and p14ARF are not 
commonly mutated or deleted in NB [3, 8, 13, 20, 21]. Additional research into the genetic abnormalities of NB is required.

\section{Cell Death}

Programmed cell death (PCD), first recognized by Lockshin and Williams as a regulated and defined process, is an essential process in normal development, cellular differentiation, and maintaining homeostasis [22-24]. The three types of PCD are apoptosis, autophagy and necrosis, otherwise known as programmed necroptosis. Differences in PCD are characterized by cellular morphology as well as protein signaling pathways. Morphologically, apoptosis is characterized by condensation of the chromatin, cell shrinkage, and blebbing of the plasma membrane. Autophagic cell death lacks chromatin condensation, but produces autophagic vacuoles within the cytoplasm. Necroptosis is associated with cytoplasmic swelling, breakdown of the cellular membrane, and moderate chromatin condensation [25-27]. Each form of programmed cell death is regulated by various signaling pathways and biochemical processes. Importantly, caspase-8 (casp8) plays a role in each type of cell death pathway.

\section{Caspase-8}

Cysteine-aspartyl specific proteases, or caspases, are the essential drivers of apoptosis. As the name denotes, caspases are proteases that target substrate-specific aspartic acid residues. The caspase family consists of 14 different caspases categorized as either inflammatory, apoptotic or having no known function $[25,28,29]$. The apoptotic subgroup is further divided into initiator or executioner caspases. Initiator caspases are the first caspases to be activated during apoptosis. Executioner caspases are activated by an initiator caspase and further transduce the apoptotic signal $[28,30,31]$ (Figure 1-1A).

The CASP8 gene located on chromosome 2q33-34 encodes for the casp8 protein $[32,33]$. Caspase- 8 contains an N-terminal prodomain comprised of two death-effector domains (DED) which bind to adaptor proteins by hemophilic interactions $[34,35]$. The C-terminal domain of casp8 consists of a large and small subunit separated by a short linker sequence [36]. The large subunit (p18) of the catalytic domain contains two essential catalytic dyads, a cysteine and a histidine (Figure 1-1B). These dyads are required for internal cleavage between subunit domains and subsequent activation of casp8. The small catalytic subunit produces the substrate-binding site $[28,30,37,38]$. In the cell, casp8 is expressed as an inactive zymogen; however, some reports have demonstrated that casp8 may have some activity in its zymogenic form $[39,40]$.

Morphological changes induced by autophagy are well characterized though poorly understood mechanistically. Research performed by Yu et al. back in 2004 showed that exposure of mouse L929 fibroblast cells to a broad caspase inhibitor known as zVAD (benzyloxycarbonylvalyl-alanyl-aspartic acid (O-methyl)-fluoro-methylketone) 
A

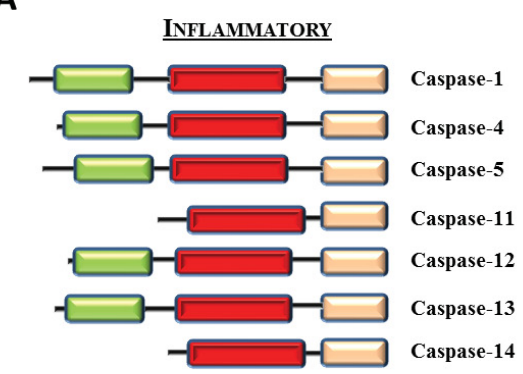

B

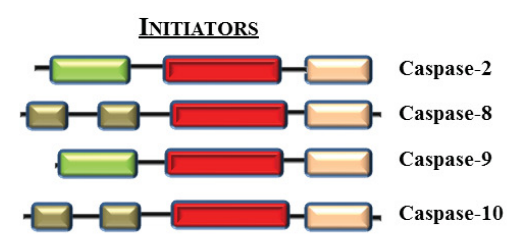

EXECUTIONER

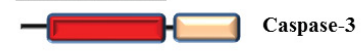

$\square$ Caspase-6
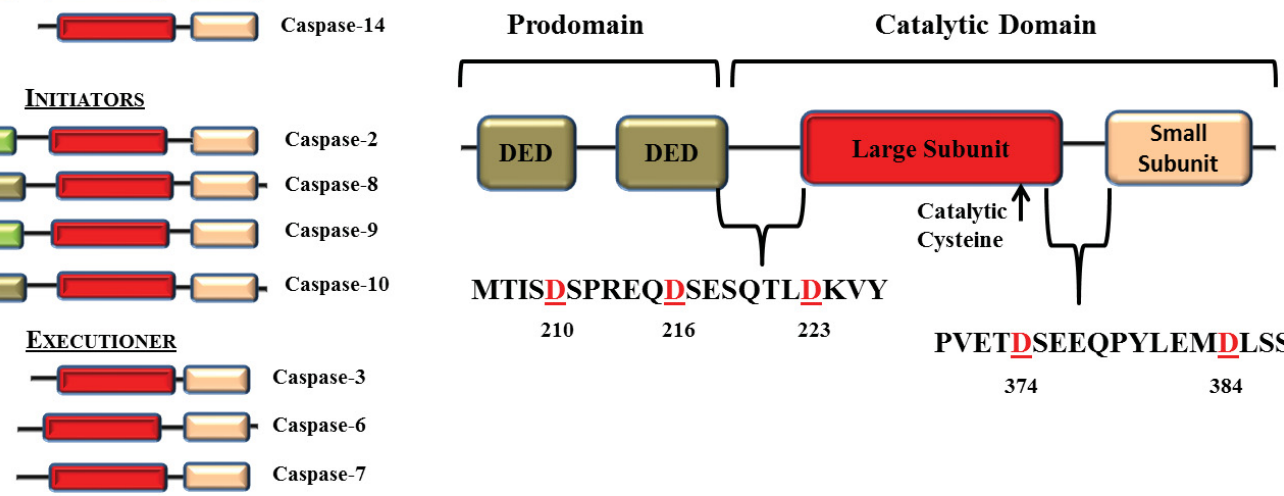

210

216

223

PVETDSEEQPYLEMDLSSP

374

384

Figure 1-1. Representation of the caspase-8 family and caspase-8.

(A) The caspase family is divided into three different subgroups based on mode of activation. Caspase- 8 is a categorized as an initiator caspase. (B) Caspase- 8 consists of an $\mathrm{N}$-terminal prodomain and a $\mathrm{C}$-terminal catalytic domain. The prodomain contains two death effector domains (DED) and the large and small subunits form the catalytic domain. The linker region between the prodomain and catalytic domain contains three cleavable aspartic acid residues. The linker region between the two catalytic subunits contains two cleavable residues [41]. 
induced autophagic cell death [42]. Yu et al. further showed that the inhibition of casp8 was critical in order to induce autophagy mediated by ATG3 (autophagy-related gene 7) and beclin-1 [42]. Validation of the involvement of casp8 in autophagy came from studies showing that casp8 mediated the cleavage and subsequent inactivation of ATG3 and beclin $1[43,44]$. During TNF-related apoptosis-inducing ligand (TRAIL)-induced autophagy, the large catalytic subunit of casp8 was found to be degraded by the autophagosome $[44,45]$. These results suggest crosstalk between apoptosis and autophagy (Figure 1-2).

Necroptosis is initiated at the plasma membrane by ligand bound death receptors (i.e. TNFR1, CD95, and TRAILR1) [26]. Briefly, TNF $\alpha$ (tumor necrosis factor) binds to the TNFR1 death receptor (tumor necrosis factor receptor 1) which then recruits the adaptor protein TRADD (TNFR-associated death domain), RIP1 (receptor-interacting protein 1), and other mediators like TRAF2/5 (TNF associated factor) and cIAP2 (cellular inhibitor of apoptosis protein) [46,47]. Internalization of the receptor bound complex promotes removal of TRAF2/5 and cIAP2 and recruitment of FADD (Fasassociated protein with death domains) and casp8. Caspase-8 recruitment and subsequent activation mediates apoptosis and blockage of necrosis through the cleavage of RIP1 and RIP3. In contrast, blockage of casp8 promotes RIP1 and RIP3 phosphorylation and formation of the necrosome comprised of TNFR1/TRADD/RIP1/RIP3/FADD [47-49] (Figure 1-2).

The apoptotic signaling pathway is initiated at either the mitochondria or plasma membrane. The intrinsic apoptotic pathway is initiated at the mitochondria by various cellular stressors. Cell death promoted at the mitochondria induces activation of caspase9 and subsequent formation of the apoptosome [50]. Death receptor-mediated apoptosis, known as the extrinsic apoptotic signaling pathway, is transduced by casp8; which is the key initiator caspase of the extrinsic apoptotic pathway [51]. Death promoting ligands (i.e. FasL and TRAIL) induce the extrinsic apoptotic pathway by binding to death receptors (i.e. FasR, DR4). Ligand binding to their respective death receptor induces receptor trimerization and subsequent recruitment of the adaptor protein known as FADD $[35,52]$. The death domain (DD) of FADD binds to the DD domain of the receptor followed by casp8 binding to the DED domain of FADD. Additional recruitment of a monomeric casp8 to FADD provides a platform for homodimerization and formation of the death-inducing signaling complex (DISC) [40,53]. Recent research suggests that dimerization and not autocleavage is essential for full catalytic activity [54-56]. Dimerization of casp8 induces a conformational change in the flexible linker region between the large and small catalytic subunits. These structural alterations reveal the active site; therefore demonstrating that uncleaved casp8 has basal levels of activity. Dimerization promotes proximity induced auto-proteolysis at specific aspartic acid residues. These cleavage events reduce steric hindrance, increase stability, and induce full catalytic activity $[39,57,58]$. Removal of the prodomain releases the catalytic subunits from the DISC into the cytosol. The large and small catalytic subunits, linked by hydrophobic interactions, undergo cleavage of the linker region between the two subunits which is thought to induce the formation of the catalytic and substrate binding sites $[30,38,57]$. Polyubiquitination of casp 8 by CUL3/RBX1 promotes $p 62$ binding 


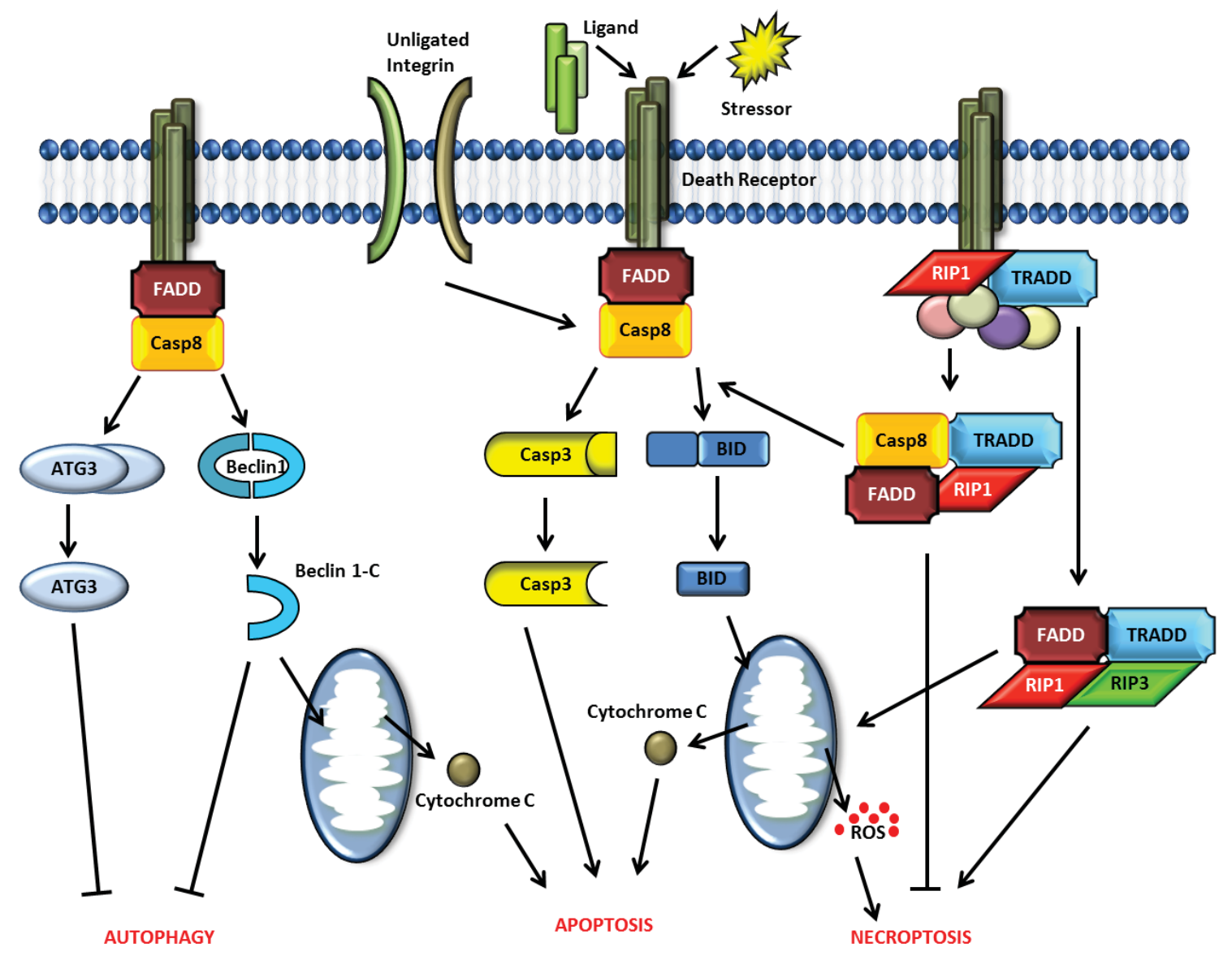

Figure 1-2. Outline of the roles of caspase-8 in various cell death pathways. This simplified overview depicts the possible roles of casp8 in cell death. Activation of various cell surface receptors or cellular stress can induce multiprotein complex formation that drives apoptosis, autophagy, and necroptosis. Induction of one death pathway over another depends on casp8 localization, expression levels of the various proteins within the complexes, and level of inhibitor proteins. Further research is required to fully understand the upstream and downstream mechanisms of these pathways and the role that casp8 plays in each. 
and translocation to cytosolic "aggresomes" (Figure 1-3). This ubiquitination and translocation enhances casp8 activity and stability [59].

The expression levels of antiapoptotic Bcl-2 proteins are thought to regulate the downstream effects of active casp8 [60]. Type I cells or low expressing antiapoptotic Bcl-2 proteins, induce casp8-mediated apoptosis through the cleavage of executioner caspases (caspase-3, 6, and 7) and PARP (poly adenosine diphosphate-ribose polymerase) [61, 62]. Cells expressing higher levels of the antiapoptotic Bcl-2 proteins, or type II cells, mediate apoptosis through the cleavage of BID by caspase-8 [63]. Truncated BID (tBID) induces mitochondrial outer membrane permeabilization (MOMP) and subsequent cytochrome c release, apoptosome formation, and cell death $[60,63]$. Importantly, casp8 has been shown to be critical in the transduction of the apoptotic signaling cascade while regulating the autophagic and necrotic cell death pathways (Figure 1-2).

Caspase-8 may also heterodimerize with cellular and viral forms of FLIP (FLICEinhibitory protein). FLIP was first identified in gamma-herpes viruses (v-FLIP) by Thorne et al, and Hu et al who later discovered a cellular homolog of v-FLIP (c-FLIP) which produces two FLIP isoforms known as c-FLIPL and c-FLIPS [64, 65]. v-FLIP and c-FLIPS both contain two DED domains yet lack the catalytic domain. Upon DISC recruitment, v-FLIP and c-FLIPS, short form, heterodimerize with casp8 and therefore, block casp8-mediated apoptosis. c-FLIPL is the longer isoform of c-FLIP, which contains two DED domains and the large and small subunits of the catalytic domain. However, c-FLIPL lacks the enzymatically essential cysteine residue within the large catalytic subunit rendering c-FLIPL catalytically inactive [66]. Initially, c-FLIPL function in cell death was controversial. Several groups demonstrated that c-FLIPL inhibited casp8-mediated cell death while others showed that it promoted casp8 activity [66-69]. Additional research showed that the role of c-FLIPL in cell death was dependent on expression levels $[70,71]$. Overexpression leads to c-FLIPL binding to all the caspase- 8 binding sites and subsequently blocking apoptosis while physiological levels promoted heterodimerization and promoted casp8 activity $[70,71]$. Interestingly, studies have revealed that the casp8/c-FLIPL heterodimer is primarily involved with prosurvival functions within the cell. Kataoka et al demonstrated that the caspase- 8 heterodimer promoted activation of the NF- $\kappa$ B pathway [72]. TNF $\alpha$-induced necroptosis via FADD-RIP1-RIP3 was blocked by casp8/c-FLIPL heterodimers [73]. Studies have shown that the casp8/c-FLIPL heterodimer contains enzymatic activity but targets different substrates. For example, the heterodimer has been shown to cleave c-FLIP, casp8, RIP, and HDAC7 as opposed to caspase-3, bid, or other proapoptotic proteins [7478]. These results demonstrate a possible mechanism that cancer cells may induce to avoid the proapoptotic function of casp8. 

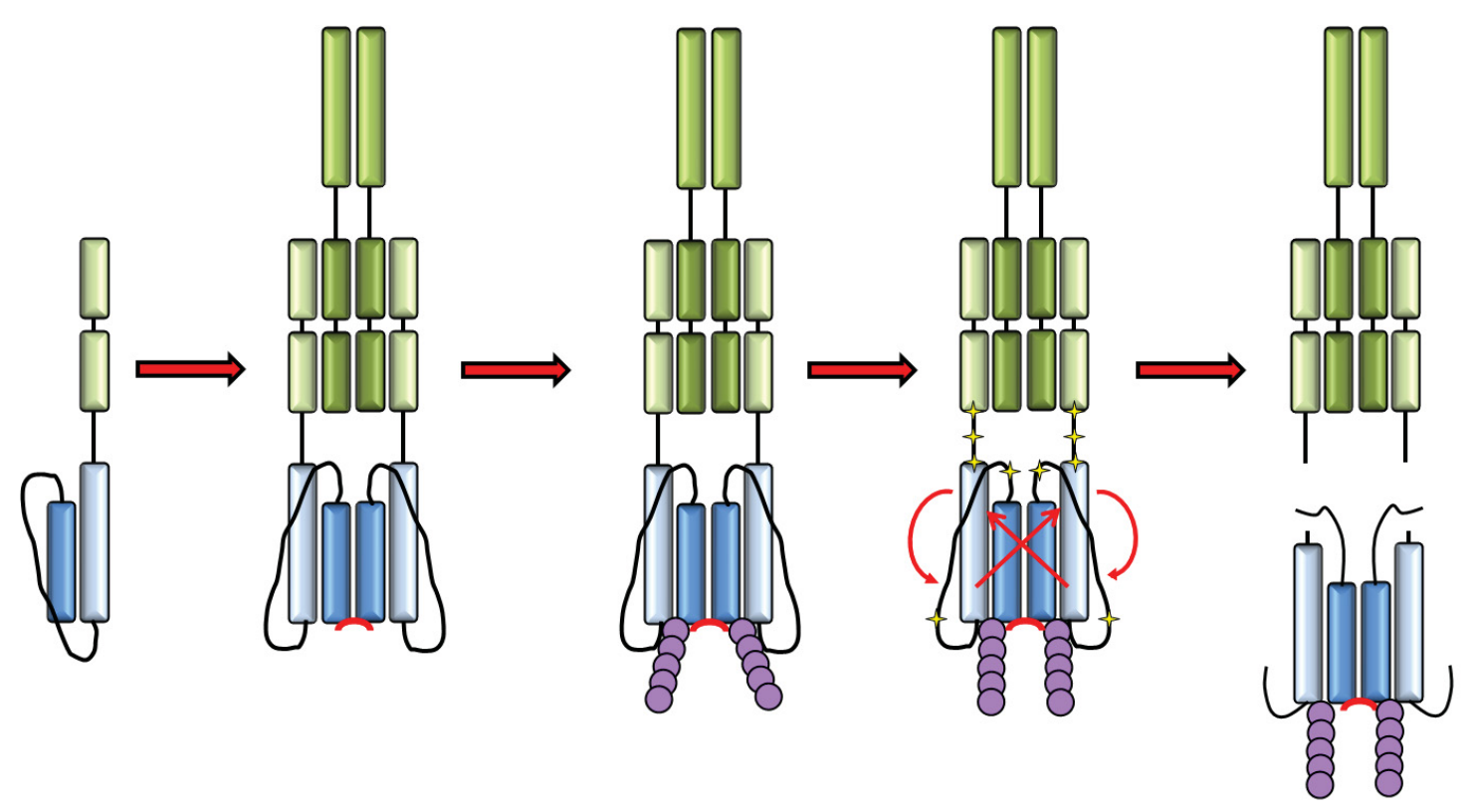

STABILITY

Catalytic ACtivity
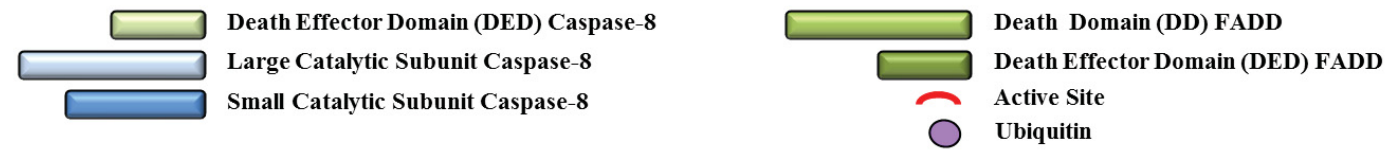

Figure 1-3. Caspase-8 mode of activation.

Caspase- 8 is maintained within the cell as an inactive zymogen. Activation of the extrinsic apoptotic pathway induces the recruitment of casp8 binding to the adaptor protein known as FADD via hemophilic interactions. Dimerization induces conformational changes of the linker region allowing for basal catalytic activity. Subsequent polyubiquitination increases caps8 stability while the autoprocessing (yellow stars) of casp8 separates its recruitment domain and the catalytic subunits. Release of casp8 from the DISC mediates casp8 translocation to cytosolic ubiquitin rich "aggresomes". 


\section{Nonapoptotic functions of caspase-8}

Ongoing research supports that casp8 is a multifunctional and multipurpose protein with often opposing roles in development and tumorigenesis (Figure 1-4). Interestingly, several of these functions are independent of the proteolytic activity of casp8 and the catalytic domain altogether (Figure 1-5). Caspase-8 has been reported to be involved in cellular growth through cell cycle regulation, proliferation, survival, and metastasis. Defects in cellular proliferation have been observed in fibroblasts, hepatocytes following partial hepatectomy, and in hematopoietic progenitor cells deficient in casp8 [79-81]. Bone-marrow cells of conditional casp8 knockout mice lacked the ability to form myeloid or B lymphoid colonies after exposure to IFN [82]. Caspase- 8 deficiency also led to a blockage of macrophage differentiation in the myelomonocytic lineage [82]. The lack of casp8 expression in endothelial cells resulted in deregulated vasculature and embryonic death [82]. Deficiency of casp8 in T cells has been associated with immunodeficiency, abolished proliferation upon antigen activation, and defects in immune homeostasis [83-85]. Furthermore, casp8 regulated the phosphorylation of ribosomal protein S6, thereby controlling the G1/S cell cycle transition [86].

Tumor cell invasiveness, migration, and adhesion have also been contributed to casp8 expression. For example, Finlay et al. demonstrated that the "RXDLL motif" within the DED domain of casp8 was crucial for the activation of ERK1/2 by epidermal growth factor (EGF) independent of the proteolytic activity of casp8 [87]. Calpain 2mediated processes such as detachment, Rac activation, lamellipodia formation, and adhesion turnover were lower in casp8 null cells suggesting that casp8 may drive calpain 2 activation [88-90]. Furthermore, integrin ligation induced Rac and Rab5 activation in a casp8-dependent manner [91]. Caspase- 8 has been shown to be involved in survival signaling pathways such as Erk1/2, phosphatidylinositol 3-kinae (PI3K), and NF- $\kappa$ B (nuclear factor kappa B). The DED domain of casp8 also has been shown to induce NF$\kappa \mathrm{B}$ activation [92]. Additional studies demonstrate that catalytically inactive casp 8 also leads to NF- $\mathrm{KB}$ activation $[93,94]$. In B cells casp8 has been observed to interact with the IKK $\alpha \beta$ (IkappaB) complex [95]. In T cells casp8 association with Bcl10, Malt1 (mucosa-associated lymphoid tissue lymphoma translocation gene), CARMA 1 (caspase recruitment domain membrane-associated guanylate kinase protein), IKK complex and TRAF6 leads to the ubiquitination and subsequent localization of casp8 to the lipid rafts [96]. Mutation of the PI3K catalytic subunit induced TRAIL and CD95L-mediated activation of NF- $\kappa \mathrm{B}$ and casp8, thereby leading to morphological changes and ROCK activation [97]. In addition, casp8 expression levels are regulated during the wound healing process. RNA levels of casp8 proximal to the wound site were found to be significantly lower than those distant from the wound. Caspase- 8 RNA levels returned to normal levels once the wound healed [98]. This data suggests that the functions of casp8 in various nonapoptotic and apoptotic pathways may be closely regulated and maintained.

It has been hypothesized that the various roles of casp 8 may be regulated by localization, involvement with various macromolecular complexes, and/or posttranslational modifications $[90,99,100]$. Cursi et al was the first to show that EGF- 


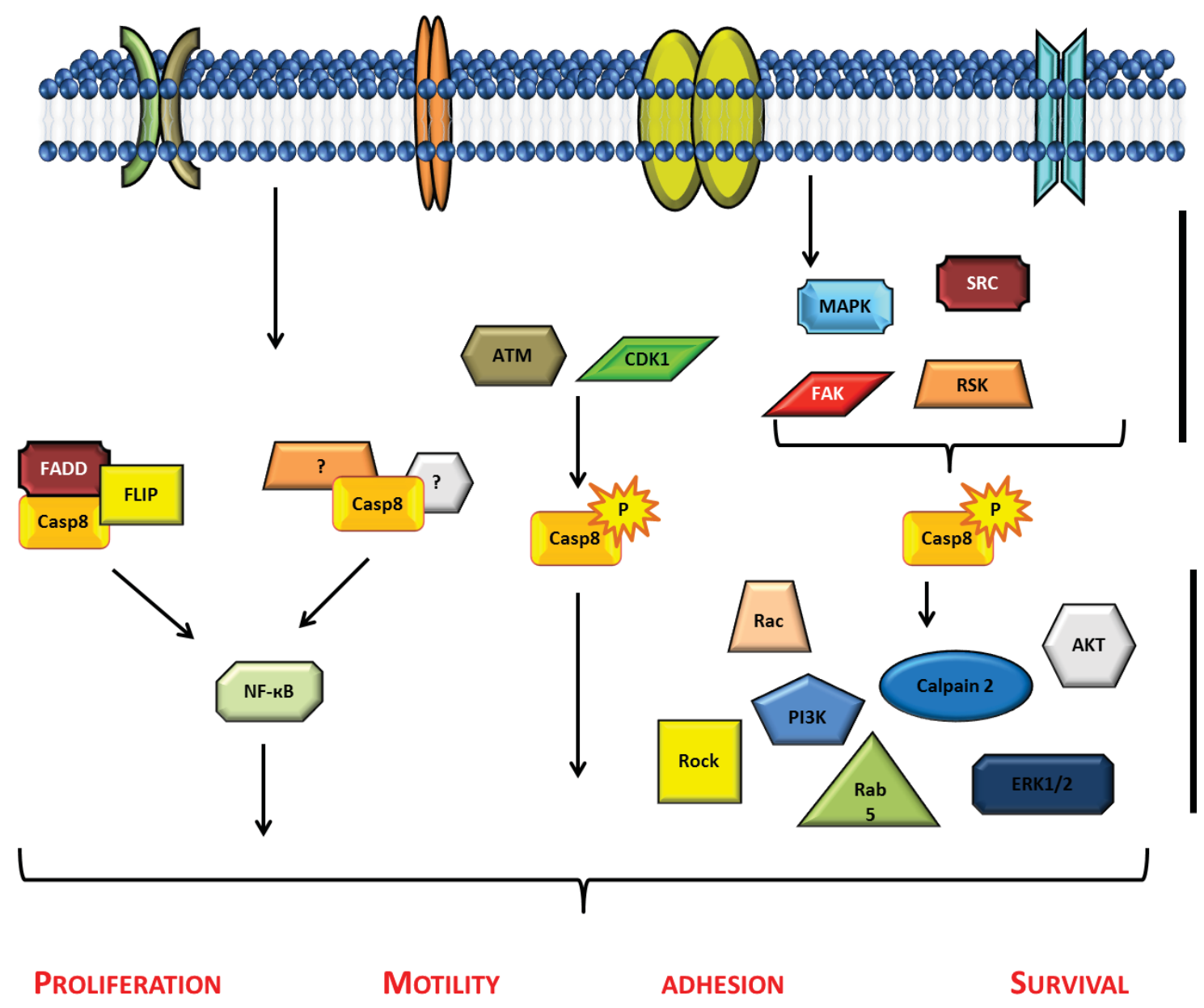

Figure 1-4. Prosurvival mechanisms of caspase-8.

Noncanonical activation of casp8 by substrate ligation to integrins, toll-like receptors, growth receptors, immune receptors, and death receptors may promote casp 8 mediated cell survival signaling. Intrinsic cellular stress may also induce casp8-mediated cell survival. Antiapoptotic functions of casp8 depend primarily on upstream activation of kinases and protein expression levels of protein complexes. The complete mechanistic functions of phosphorylated casp8 and protein-protein formations are unclear and further research is needed. 


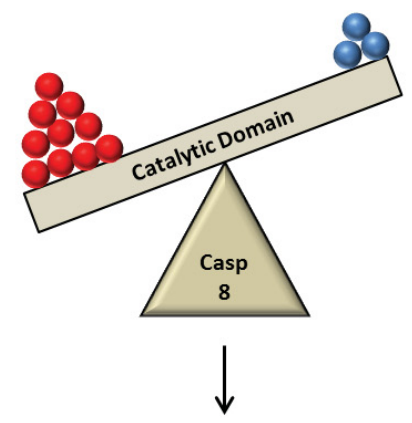

- Induce apoptosis

- Regulate autophagy

- Promote NF-KB

- Localization in nucleus

- Induces invasion via ROCK cleavage

- Hematopoietic progenitor cell growth

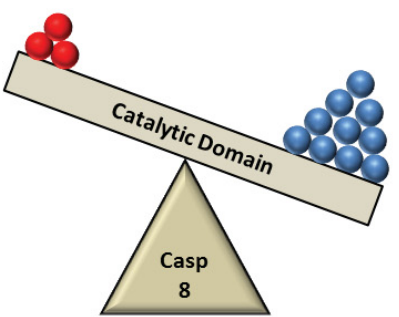

$\downarrow$

- Activate Rab5, Rac, and Calpain 2

- EGFR-mediated migration

- Localization in nucleus and mitochondria

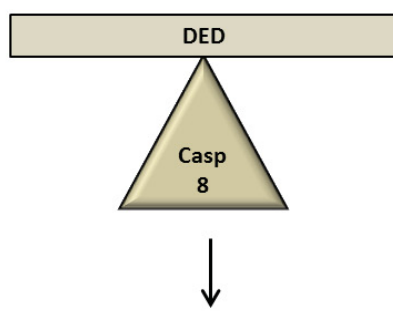

- Binds to perinuclear microtubules

- Induce differentiation

- Localization to nucleus

- Src binding

Figure 1-5. Domain specific functions of caspase-8.

The scheme depicts the possible functions induced by either the catalytic domain or death effector domain (DED) of casp8 in response to catalytic stimuli thereby required the catalytic activity (red dots) casp8. In contrast, the catalytic domain of casp8, independent of its catalytic capabilities, may activate various proteins, translocate, and/or induce cellular motility. The DED domains contain no proteolytic capabilities; however, DED domains independent of the $\mathrm{C}$-terminal catalytic domain may promote various signaling pathways. 
mediated migration induced casp 8 phosphorylation by Src at tyrosine 380 [101]. This phosphorylation induced casp8 interactions with the $\mathrm{p} 85$ subunit of the phosphatidylinositol 3-kinase thereby increasing migration and adhesion. Later, Barbero et al. demonstrated that integrin ligation could also induce casp 8 phosphorylation at tyrosine 380 and promote the localization of casp 8 to the lamellae of migrating cells [102]. In addition, Cursi et al. further demonstrated that the phosphorylation event at tyrosine 380 suppressed the catalytic activity of casp8 [101]. Caspase-8 phosphorylation by cyclin-dependent kinase 1 (Cdk1) and ribosomal S6 kinase 2 (RSK2) at serine 387 and threonine 263 respectively, lead to a decrease in Fas-mediated apoptosis [103, 104]. Furthermore, p38MAPK phosphorylation of casp8 at serine 347 also suppressed the catalytic activities of casp8 [105]. The DNA damage repair protein, ataxia telangiectasia mutated (ATM) kinase was reported to phosphorylate casp8 at serine 219 upon double strand DNA breaks [106]. In cancer, several of these kinases are reported to be upregulated due to their pro-survival function; hence, they may contribute to altering the balance between the canonical function of caspases to a more noncanonical or prosurvival function [107].

Primarily, casp8 was considered as a cytoplasmic protein due to its role in apoptosis; however, research reveals that, in neurons, the active form of casp8 (p46) may enter the nucleus [108]. In human fibroblasts and mouse striatal cells, casp8 is shown to be localized primarily in the mitochondria and partially in the nucleus [109].

Furthermore, active casp8 (p18/p10) cleaves PARP2 within the nucleus during ischemiainduced apoptosis [110]. The DED domains bind to the perinuclear microtubules during paclitaxel-mediated apoptosis and during differentiation of skin and myeloid cells [111, 112]. Besnault-Mascard et al. suggested that sumoylation of casp8, at lysine 156 induced nuclear localization [113]. As previously stated, casp8 may localize at the lamella and lipid rafts [96, 102]. Collectively, this data strongly suggests that cell type and cellular conditions may influence casp8 function and localization.

\section{Caspase-8 in cancer}

Defective apoptotic machinery, a common characteristic of cancer cells, is associated with tumorigenesis and therapeutic resistance [114]. Teitz et al. and others have demonstrated that casp8 is preferentially silenced in NB cell lines and patient samples though rarely deleted [17, 115-117]. Loss of casp 8 has also been shown in other tumors including small cell lung carcinoma, rhabdomyosarcoma, glioblastoma, retinoblastoma, medulloblastoma, Wilms tumors and colorectal carcinomas [118-123].

Suppression of casp8 expression resulted from the hypermethylation at the exon 3 -intron 3 region, upstream of the protein translation start site [17]. A study of 180 pediatric tumors revealed that the exon 3-intron 3 region of the CASP8 gene was methylated in $83 \%$ of rhabdomyosarcomas, $81 \%$ medulloblastomas, $59 \%$ retinoblastomas, 52\% neuroblastomas, and 19\% in Wilms tumors [118]. The hypermethylation of casp 8 showed a statistically significant correlation with relapse susceptibility, a decreased overall survival rate, and an increase in resistance against 
death receptor-mediated apoptosis and some chemotherapeutics [116, 123-127]. Studies demonstrated that by using demethylating reagents such as, gamma interferon $(\gamma$-IFN), 5'-aza-2-deoxycytidine, or retinoic acid, casp8 expression could be restored [127-130].

In addition to methylation, casp 8 deficiency may also contribute to protein mutation, though to a lesser extent. Only $5 \%$ of colorectal carcinomas and $13 \%$ of hepatocellular carcinomas exhibited mutated casp8. To date, there have been five reported mutations of casp8: a frameshift mutation at position 1381, three missense mutations (R413G, Q366H, and R162I), and one nonsense mutation resulting in a truncated form of casp8 [120, 122, 131].

The role of casp8 in tumorigenesis and overall patient survival remains unclear. For example, Strater et al. reported that casp8 expression was correlated with a decrease in the long-term survival rate of patients with colon carcinoma [132]. In head and neck squamous cell carcinoma (HNSCC) it was shown that casp8 expression was correlated with a better prognosis; although, HNSCC with lymph node metastasis casp8 expression was significantly associated with a poorer survival rate and outcome [133]. This data suggests that the balance between the various functions of casp 8 may be altered based on tumor type and the stage of tumor growth.

Several strategies have been developed to upregulate casp 8 and promote casp 8 as a potential therapeutic target for cancer therapy. As previously stated, due to the high frequency of casp8 methylation in cancers, one possible method consists of using the demethylating agent, 5-aza-2'deoxycytidine (5-Aza) [116, 130]. This treatment increased casp8 expression in several tumors presenting with epigenetically silenced casp8 including NB [134-136]. Furthermore, the casp8 promoter region contains interferonsensitive response elements that regulate casp8 expression $[137,138]$. Accordingly, exposure to interferon- $\gamma$ (IFN- $\gamma$ ) increased casp8 expression and sensitized cells to IR and death receptor-mediated cell death $[139,140]$. Successful studies using IFN- $\gamma$ was shown in an in vivo Ewing sarcoma xenograft model [139]. A phase I clinical trial using IFN- $\gamma$ to treat NB patients demonstrated that IFN- $\gamma$ increases casp8 expression. Combination of both IFN- $\gamma$ and 5-Aza demonstrate significantly enhance casp8 expression and apoptotis of cancer cells upon treatment with TRAIL [130]. Retinoic acid (RA) has also been shown to induce casp8 transcription via a CREB binding site on the CASP8 gene [127]. Though inducing casp8 expression may present an attractive therapeutic target, it may also promote survival. Research shows that casp8 expression can be elevated in some tumors and some malignant tumors may tolerate high levels of casp8. Furthermore, given the intense selective pressures present in the tumor microenvironment, the upregulation of casp8 by demethylating agents may promote these noncanonical nonapoptotic prosurvival roles of casp8. In such situations, increasing casp8 may be detrimental [141, 142]. 


\section{Role of caspase-8 in neuroblastoma}

Interestingly, although casp8 expression is silenced in approximately $50-70 \%$ of NB patient samples, there are conflicting reports regarding the loss of casp 8 in aggressive NB. The most compelling results came from the work performed by Fulda et al. who analyzed a total of 162 patient samples. This study demonstrated that there was no significant correlation between the loss of casp8 and MYCN amplification or poor prognosis [143]. Results from Takita et al. revealed that 11 of 15 NB cell lines silenced casp8 expression, but no correlation was made regarding MYCN amplification [144].

To determine the role of casp8 in NB tumorigenesis and metastasis our lab used isogenic NB7 cells transduced with physiological levels of casp8, which have genetic deletions of both casp8 alleles, and engraftment studies performed in SCID mice injected by tail vein (not published). Interestingly casp8 expressing cells formed more and larger tumors in comparison to controls. In addition, tumor size was also significantly larger in casp8 expressing cells. Paradoxically, casp8 expression in the tumors was decreased. These results suggest that casp8 provided some initial benefit for tumor establishment though its down-regulation was required in order to survive. Therefore, casp8 may have additional nonapoptotic or noncanonical roles in tumorigenesis, such as increased cell adhesion and migration. Confirmation of the potential role of casp8 in tumor cell metastasis was performed by Stupack et al. in collaboration with our lab [145]. The chick embryo assay was used to determine the metastatic potential of NB7 cell lines seeded onto the chick chorioallantoic membrane (CAM). These studies revealed no significant difference of tumor size between NB7 cells with or without casp8. NB7 cells, absent for casp8 expression exhibited an increased metastatic potential. In contrast, other research groups demonstrated that casp 8 expression significantly enhances metastasis and may promote tumor growth as previously mentioned [88, 89, 146, 147]. In addition, Stupack et al. found that apoptotic cells were located at the tumor periphery in casp 8 positive tumors suggesting that cells expressing casp 8 within a foreign environment underwent apoptosis [145]. Previous research revealed that casp8 association with integrins on the cell surface could mediate a process known as integrin-mediated death (IMD). Therefore, NB7 cells were sorted for high and low integrin expression ( $\alpha 3 \beta 1)$. NB7 cells expressing casp8 and integrin at low levels exhibited a higher metastatic rate and decreased levels of apoptosis at the tumor periphery. In contrast, NB7 cells expressing physiological levels of casp8 and high levels of integrin did not metastasize and underwent apoptosis at a higher frequency. Tumors from SCID mice and human patient samples revealed a decrease in expression level of $\alpha 3 \beta 1$ and casp8 suggesting a more aggressive phenotype. Advanced stage NB (stage 3 and 4) showed a decrease in $\alpha 3$ integrin mRNA [145].

In comparison to normal cells, tumor cells are subject to different stressors such as hypoxia, radical oxygen species (ROS), starvation, genotoxic insults, and mechanical stress. These stressors promote extracellular matrix (ECM) stiffness, increased survival, and integrin activation. The most common biological results of ECM-cell interaction is adhesion, invasiveness, and migration. This implies that when casp8 levels are low a decrease in integrin function may result in cell detachment [148]. 
Our lab was the first to demonstrate a role for casp8 in metastasis. Additional research has validated and further clarified casp8 involvement in metastasis $[88,89,97$, 146, 149]. These experiments were performed using various in vitro matrices; isogenic cell lines transduced with physiological levels of casp8, and engraftment studies in immunocompromised mice by tail vein and flank injection. In all studies performed, casp8 was validated to have a key role in survival, migration, attachment, and metastatic capabilities. Caspase- 8 was also shown to be down-regulated at secondary metastatic sites. In contrast, these results also suggest that the loss of casp 8 may promote detachment from the primary mass $[88,89,102,146,149]$.

This data suggests that independent of casp8 expression the overall primary tumor growth is relatively unaffected. Caspase- 8 expression in the primary tumor may offer a selective advantage for cellular invasion and metastasis in that casp8 expression enhances cellular attachment and motility. Paradoxically, expression of casp8 must be silenced in order to avoid IMD in the secondary site (Figure 1-6).

To understand the role of casp8 in NB, Teitz et al tested the in vivo role of casp8. To overcome the embryonic lethality of casp 8 deficiency in mice, they crossed the THCre mouse; which expresses the Cre recombinase under the tyrosine hydroxylase $(\mathrm{TH})$ promoter at day E9.5, with floxed casp8 mice $[83,150]$. Caspase-8 deficiency resulted in no tumor formation until these mice were crossed to TH-MYCN mice $[151,152]$. As previously stated, MYCN amplification occurs in approximately $30 \%$ of advance stage NB. Loss of casp8 in mouse neural crest cells with MYCN amplification significantly increased bone marrow metastasis; though no significant difference was observed in primary tumor growth [152].

\section{Specific Aims}

Caspase- 8 is silenced in up to $70 \%$ of advanced-stage NB patient tumors and the biological consequences of casp8 deficiency results in enhanced metastatic cell survival by preventing integrin-mediated cell death and decreased cell sensitivity to chemotherapeutic drugs in NB [127, 128, 145, 153]. On the other hand, casp8 expression is maintained in $30-50 \%$ of NB and promotes ruffle and lamellipodial formation, enhanced cell migration, and adhesion [88, 91, 149]. These processes were determined to be independent of the enzymatic activity required for apoptosis. Caspase- 8 may also regulate cell cycle progression via the S6K pathway, NF-кB activation, and Erk1/2 activation $[86,87,96,101]$. Previously, kinases including Src, ATM, p38 MAPK and Cdk1 have been shown to modulate and regulate casp8 activity and cellular function via phosphorylation. In general, phosphorylation reduces the enzymatic activity of casp8. In hepatocellular carcinoma and colon cancer elevated levels of phosphorylated casp8 were observed, suggesting that the phosphorylation of casp 8 may enhance tumorigenesis and/or metastasis. Overall, casp8 appears to be a multi-functional protein playing key roles in two opposing processes, cell death and survival. This phenomenon raises the question as to how a single protein can initiate opposing functions within the cell. The studies of this dissertation are designed to examine the hypothesis that casp 8 has pro- 

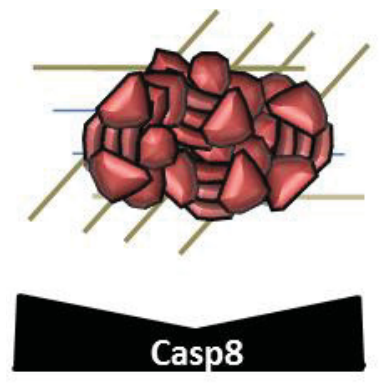

PRIMARY
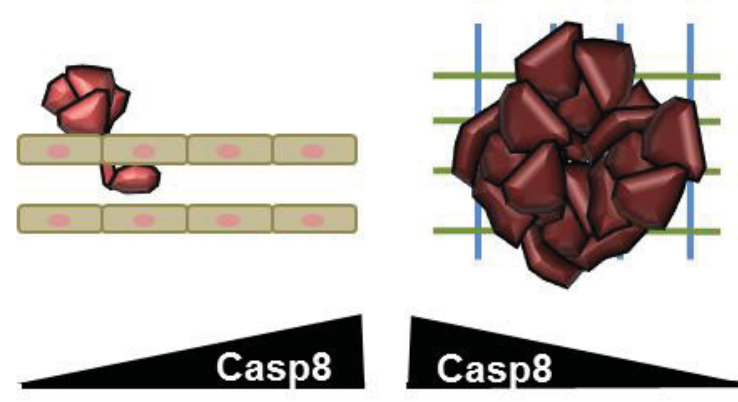

Casp8

METASTASIS SECONDARY

Figure 1-6. Role of caspase-8 expression levels promote tumorigenesis and metastasis.

Caspase-8 expression within the primary tumor promotes solid tumor formation. Continued expression of caspas 8 drives tumor growth producing larger tumors than casp8 deficient tumors. However, casp8 positive tumors have a larger number of apoptotic cells along the tumor periphery. In vitro studies demonstrate that casp 8 positive cells have an increased migration and invasive potential. Tumors located at secondary tumor sites have silenced casp 8 expression suggesting that casp 8 may promote survival at the primary tumor site while inducing cell death in foreign microenvironments. 
death and pro-survival functions during NB tumorigenesis and that post-translational modification of casp8 may affect the balance of casp8 between these two opposing pathways. To test this hypothesis, I propose the following specific aims.

Aim 1: To determine whether casp8 expression is essential in NB tumorigenesis. Approximately, $30-50 \%$ of NB patient samples maintain casp8 expression suggesting that casp8 may possess noncanonical prosurvival functions. Studies in this aim will:

a) Examine the biological effect of casp8 expression in NB tumorigenesis.

b) Determine the biological role of the catalytic activity of casp8 in NB tumorigenesis.

Aim 2: To determine whether the modulation of casp8 by phosphorylation promotes critical nonapoptotic functions during NB tumorigenesis. Previous research showed that ATM could phosphorylate casp8 at serine 219 upon ATM activation by DNA damaging agents. Studies in this aim will:

a) Determine if DNA damage induced by irradiation leads to casp8 phosphorylation.

b) Confirm that this modulation of casp8 is dependent on ATM activation.

c) Establish the biological significance of phosphorylated casp8 on apoptotic and survival pathways.

d) Identify the effect of casp8 phosphorylation in NB tumorigenesis. 


\section{CHAPTER 2. CASPASE-8 PROMOTES NEUROBLASTOMA TUMORIGENESIS}

\section{Introduction}

Neuroblastoma (NB), a childhood cancer of the sympathetic neural crest cells, accounts for $7-10 \%$ of infant malignancies and $15 \%$ of cancer-related deaths in children $[3,154]$. Advancements in chemotherapies have increased pediatric cancer-survival rates to more than $75 \%$ while long-term survival rates of patients with advanced-stage NB still remain at $\sim 30 \%$ [155]. Hallmark genetic abnormalities associated with the poor outcome of high-risk NB include MYCN amplification, deletion of 1p, 11q, and gain of 17q [3, 13, $17,125,126]$. Defective apoptotic machinery is also commonly associated with tumorigenesis and therapeutic resistance [114]. In NB, approximately 50-70\% of patient tumor samples exhibit a loss of casp8 expression, indicating that casp8 may play an important role in tumorigenesis [17]. Other tumors including medulloblastoma, retinoblastoma, rhabdomyosarcoma, and small cell lung carcinoma have also been shown to silence casp8 expression $[118,119,121]$. Downregulation of casp8 is frequently attributed to the hypermethylation of $\mathrm{CpG}$ islands within the CASP 8 promoter region [117]. Research suggests that the loss of casp8 in NB significantly enhanced tumor cell metastasis and survival at secondary sites by inhibiting integrin-mediated cell death (IMD) $[88,145,156]$. Loss of casp 8 in NB decreased cell sensitivity to chemotherapeutic drugs $[127,128,145,153]$.

Caspase-8, located on chromosome $2 \mathrm{q} 33-34$, is the initiator caspase of the extrinsic apoptotic signaling pathway $[28,117]$. Caspase- 8 is composed of a proteinprotein interacting $\mathrm{N}$-terminal domain and a catalytic $\mathrm{C}$-terminal domain; comprised of a large and small subunit separated by a short linker sequence [36]. Ligand binding to its respective death receptor promotes receptor trimerization and recruitment of the adaptor protein, FADD. The prodomain of casp8, comprised of two death effector domains (DED), interacts with the DED domain of FADD. Recruitment of an additional casp8 to FADD allows for casp8 homodimerization; thereby promoting self-cleavage events at key aspartic acid residues within the monomers. These auto-cleavage events relieve steric hindrances, increase stability, and induce full activation. Interestingly, dimerization is mandatory for full catalytic activity, not auto-cleavage $[54-56,157]$. The downstream effects of receptor-mediated casp8 activation is mediated through the activation of executioner caspases (caspase-3 and caspase-7) and/or BID cleavage [56, $63,158,159]$.

Caspase-8 has long been considered as primarily a transducer of cell death; however, ongoing research has clearly demonstrated casp8 to be a multifunctional and multipurpose cellular protein. Caspase- 8 has been reported to be involved in cell migration [89, 146, 149], adhesion [88], differentiation [160, 161], proliferation [147, 162], cell cycle regulation [86], wound healing [98], NF-אB and ERK $1 / 2$ signaling [87, 94], and Rock activation [97]. Several of these functions are independent of the catalytic activity of casp8 and are functionally dependent on specific domains of casp8. 
Interestingly, $30-50 \%$ of NB patient samples maintain casp 8 expression. This phenomenon drives the question as to whether casp 8 may be providing a survival advantage in this subset of tumor samples, and if so, how is the catalytic activity of casp8 regulated to promote survival in contrast to cell death. Prosurvival mechanisms of casp8 have been shown to promote ruffle and lamellipodial formation, enhanced cell migration, and adhesion $[88,91,149]$. In addition, casp8 is linked to cell cycle regulation via the S6K pathway, NF-кB activation, and Erk1/2 activation $[86,87,96,101]$. These processes were determined to be independent of the enzymatic activity required for apoptosis. Caspase- 8 deficient mice present with severe developmental abnormalities leading to death at E10.5, suggesting that casp8 plays a significant role during embryogenesis. The lethality of casp 8 knockout can be rescued by rendering casp 8 catalytically inactive by a single point mutation of an essential aspartic acid residue, D387 [163]. These results show that casp8 plays a key prosurvival role during normal development that is independent of its proteolytic, cell death inducing, property. Furthermore, work performed in keratinocytes reveals that casp8 expression is regulated during wound healing indicating that casp8 is closely regulated. Overall, these studies demonstrate that casp 8 is a multifunctional protein with both proapoptotic and prosurvival functions, and in order for cells to survive the enzymatic activity of casp8 must be controlled.

Studies performed in fertilized chick eggs revealed that casp8 expression in NB cells did not significantly enhance primary tumor growth [145]. This work demonstrated for the first time that casp 8 may act as a metastasis suppressor gene since data showed that cells expressing casp8 were more prone to undergo apoptosis in a foreign environment. Conditional knockout of casp8 in mouse neural crest cells showed similar results regarding primary tumor formation and an increase in bone marrow metastasis [152]. In contrast, a study comprised of the largest cohort of NB patient samples revealed that casp8 is not preferentially lost in advanced stage-NB. Furthermore, in vitro analysis of casp8 shows that casp8 expression enhances metastasis; suggesting a potential survival function of casp8 in NB demonstrating why up to half of NB patient samples maintain casp8 expression. Taken together, these studies demonstrate that little is understood about the exact mechanism and biological role of casp8 in NB.

In this study, we hypothesized that casp8 expression is essential in NB

tumorigenesis. We examined the role of casp8 to promote cell survival and tumorigenesis as determined by our novel orthotopic xenograft model. For the first time, we report that casp8 expression significantly enhanced tumor growth, tumor establishment, and decreased overall survival in NB transplanted mice. Furthermore, we demonstrate that the catalytic activity of casp 8 is not required for its protumorigenic function of primary tumors and that the $\mathrm{N}$-terminal prodomain of casp 8 is essential in casp8-mediated tumor growth. We report in our tumor xenografts derived from casp8 positive cells that casp8 expression is decreased. A model summarizing our findings is proposed that casp8 may be essential for engraftment of our NB cell line in our xenograft models. Increased engraftment promotes tumor growth and development. At later stages of tumor development casp8 is downregulated; thereby, decreasing tumor cell death. 


\section{Results}

\section{Caspase-8 expression promotes NB tumor growth in low tumorigenic cells}

To examine the potential biological role of casp8 in NB and explain why casp8 is maintained in 30-50\% of NB patient samples we employed two isogenic cell lines, NB7 and NB1691, both deficient for casp8 expression. Both cell lines were transduced with wild-type (wt) casp8 and selected for GFP (green fluorescent protein) positive cells using flow cytometry. Overexpression of casp8 drives cell death; therefore, casp 8 was expressed at relatively physiological levels. We compared the reconstituted expression levels of casp8 in NB7 and NB1691 cells to four neuroblastoma cell lines expressing endogenous casp8 to validate expression levels (Figure 2-1A). Endogenous casp8 is often expressed as two isoforms, represented as a doublet by western blot analysis [164]. These isoforms only vary by a few amino acids at the N-terminal and have been shown to have similar functions and rates in promoting apoptotic cell death [164]. Our NB cell lines were reconstituted with the larger $55 \mathrm{kDa}$ isoform of casp8, recognized as the upper band. Densitometric analysis of reconstituted casp8 compared to both isoforms supported that casp8 expression is relatively equivalent to endogenous casp8 (data not shown). Cell lines reconstituted with wild-type casp8 will be indicated as C8 (NB7-C8wt and NB1691-C8wt) throughout the study.

Approximately, $40 \%$ of NB patient samples develop in the para-adrenal or intraadrenal region [7]. To represent this event in a mouse model, we developed a novel, minimally invasive, ultrasound-guided orthotopic xenograft mouse model [165]. This method results in low morbidity and mortality, and has a high tumor engraftment success rate. Histological examination of xenografts derived from this orthotopic model expressed many of the key hallmarks of neuroblastoma including PGP 9.5, synaptophysin, neuron specific enolase, and tyrosine hydroxylase [165].

To monitor in vivo tumor growth by live imaging, NB7-C8wt and NB1691-C8wt cells (GFP positive) were transfected with luciferase and selected for stable expression using YFP (yellow fluorescent protein) or zeocin resistance, respectively. NB cell lines with and without casp8 were orthotopically injected into the para-adrenal region of nude mice. NB7 and NB1691 cells expressing empty vectors (NB7-vec or NB1691-vec) were used as negative controls. Tumor growth was monitored weekly by bioluminescence. NB7 cells with and without casp8 demonstrated relatively consistent tumor growth until week seven. Between the seven and ten week time points mice reached tumor burden and were euthanized in casp8 positive NB7 derived tumors. A ten week time frame for tumor burden is similar to other mouse models; namely tail vein injected xenografts of NB7 cells and the TH-MYCN mouse with and without conditional casp8 knockout. NB1691 cells with and without casp8 were more tumorigenic and produced tumors as early as three weeks, precluding assessment of casp8 in promoting tumor growth in this model. To control for varying expression levels of luciferase in the NB cell lines, luminescent values at the weekly time points were normalized to the first week time point for each respective mouse. A two log increase in tumor cell growth from the first week 
A

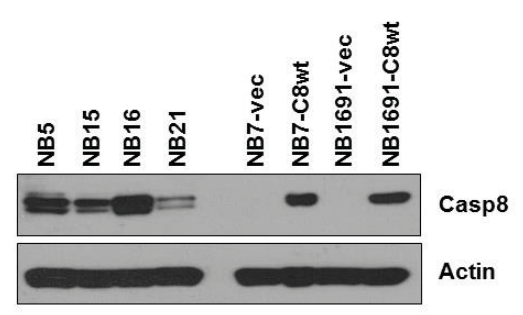

C

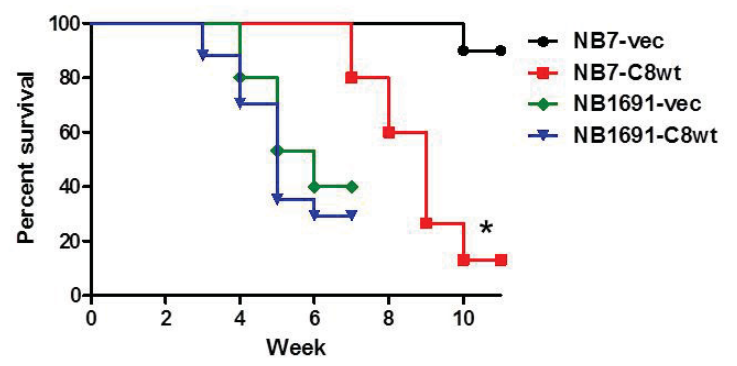

B

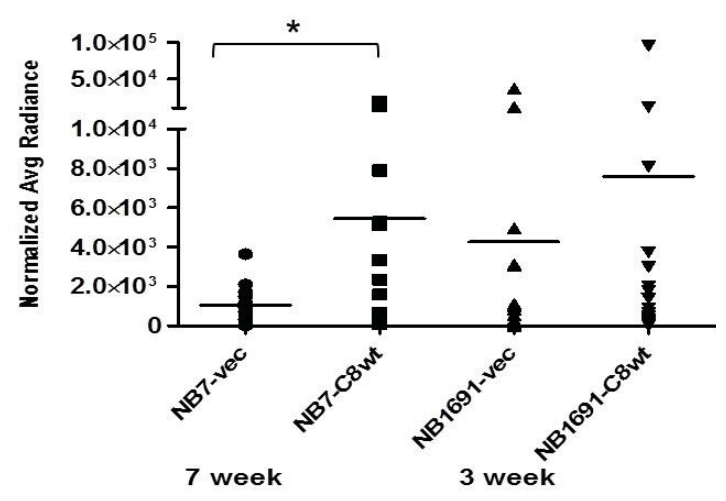

D

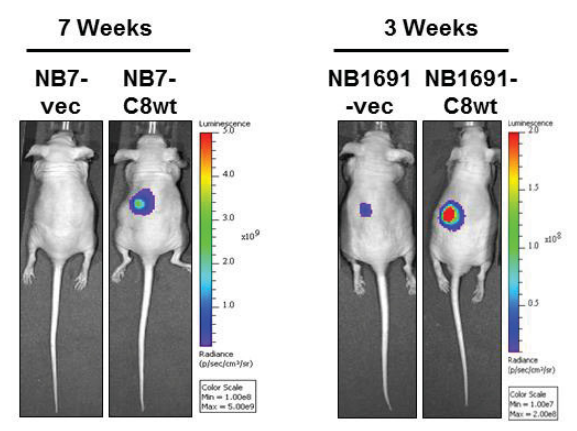

Figure 2-1. Caspase-8 increased neuroblastoma tumorgenicity.

(A) NB7 and NB1691 cells were reconstituted with endogenous levels of capase-8 (C8) wild-type (wt) and compared to the endogenous levels of casp8 in various NB cell lines. (B) NB cells with and without casp8 (vector control), as indicated, were orthotopically injected into the para-adrenal region of nude mice. Mice were analyzed one week post injection to confirm injection and establish a baseline for tumor growth. Graph represents normalized bioluminescent at the 7 week time point. $(*, P \leq 0.005)$ (C) Survival plot of tumor bearing mice. Mice with tumors exceeding a two log increase in bioluminescence were removed from study. ( $\left.{ }^{*}, \mathrm{P}<0.001\right)$ (D) Representative bioluminescent images of tumor bearing mice. Error bars, \pm s.e.m. 
luminescent radiance value was predetermined by experimental data as tumor burden or approximately $10 \%$ of normal body weight. NB7 cells with and without casp 8 showed statistically significant differences by week seven $(\mathrm{P}<0.005)$ and maintained until the ten week time point $(\mathrm{P}<0.004)$ (Figure 2-1B). The more tumorigenic cell line, NB1691, demonstrated no significant difference in luminescence between expression of casp8 and vector only control (Figure 2-1B). Comparison of vector controls to casp8wt expressing NB7 cells revealed that casp8 significantly increased tumor growth and decreased survival from $90 \%$ in control cells to $13 \%$ by the end of week ten $(\mathrm{P}<0.001)$

(Figure 2-1C). Figure 2-1D is a representative bioluminescent image of mouse xenografts derived from both NB cell lines expressing either vector control or casp8 wt. Mice were analyzed using the same radiance scale with a range between $1 \times 10^{8}$ to $5 \times 10^{9}$ or $1 \times 10^{7}$ to $2 \times 10^{8}$ photons $/ \mathrm{sec} / \mathrm{cm}^{2} / \mathrm{sr}$ for NB7 and NB1691 cell lines, respectively (Figure 2-1D).

\section{Caspase-8 increased tumor engraftment and growth in NB7 cells}

After the ten week time point, mice not having reached tumor burden were euthanized and visually analyzed for tumor presence. All mice enrolled in the study had been validated two weeks post injection for successful transplantation by live imaging. Interestingly, we observed that NB7 cells lacking casp8 exhibited an orthotopic engraftment rate of only 53\% (19/36). On the other hand, casp8 positive NB7 cells demonstrated a significantly higher engraftment rate of 96\% (21/22) (Figure 2-2A). NB7 cells without casp8 maintained luminescent signaling but lacked solid tumor establishment, suggesting that the cells were present within the abdominal cavity although unable to establish solid tumors. NB1691 cells, independent of casp8 expression, were able to establish solid tumors at seemingly similar rates of $67 \%$ in vector control cells and 76\% in casp 8 positive cells (Figure 2-2B). The results presented in Figure 2-2 $\mathbf{A}$ and $\mathbf{B}$ represents only the presence or absence of a solid tumor mass and are unbiased based on tumor size. Since all mice involved in the study were analyzed by bioluminescence to confirm successful engraftment prior to study enrollment, this would suggest that casp8 expression significantly enhances cell colonization and tumor establishment. Visual analysis of NB7 casp8wt tumors produced significantly larger tumors than the casp8 deficient NB7 derived tumors (Figure 2-2C). Volumetric analysis by ultrasound and calipers measurements of tumor size was difficult due to the involvement and often the engulfment of the kidney, adrenal, and other possible organs by the NB cell lines. We further noticed that the casp8 positive NB7 tumors were seemingly more angiogenic, although histological examination of CD31, an endothelium marker, revealed no significant difference between vector controls and casp8 positive cells. The parental NB7 cell line was also tested in our orthotopic model to address potential effects contributed by the casp8 expression vector. After ten weeks, mice were euthanized and any visible tumors removed. Comparison of tumor size between NB7 parental and vector control lines demonstrated that the tumorigenicity of NB7 cells is not affected by the plasmid and is therefore in response to casp8 expression. In contrast to NB7-C8wt cell line, NB7-vec control cells formed small localized tumors easily removed from the para-adrenal region, suggesting a proliferative advantage for casp8 positive 

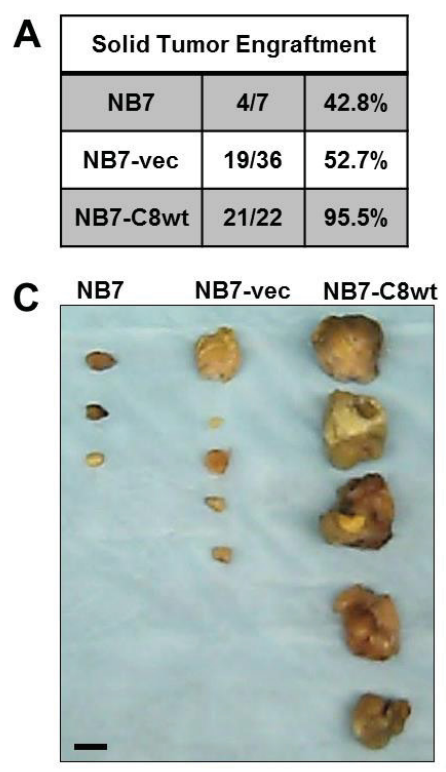
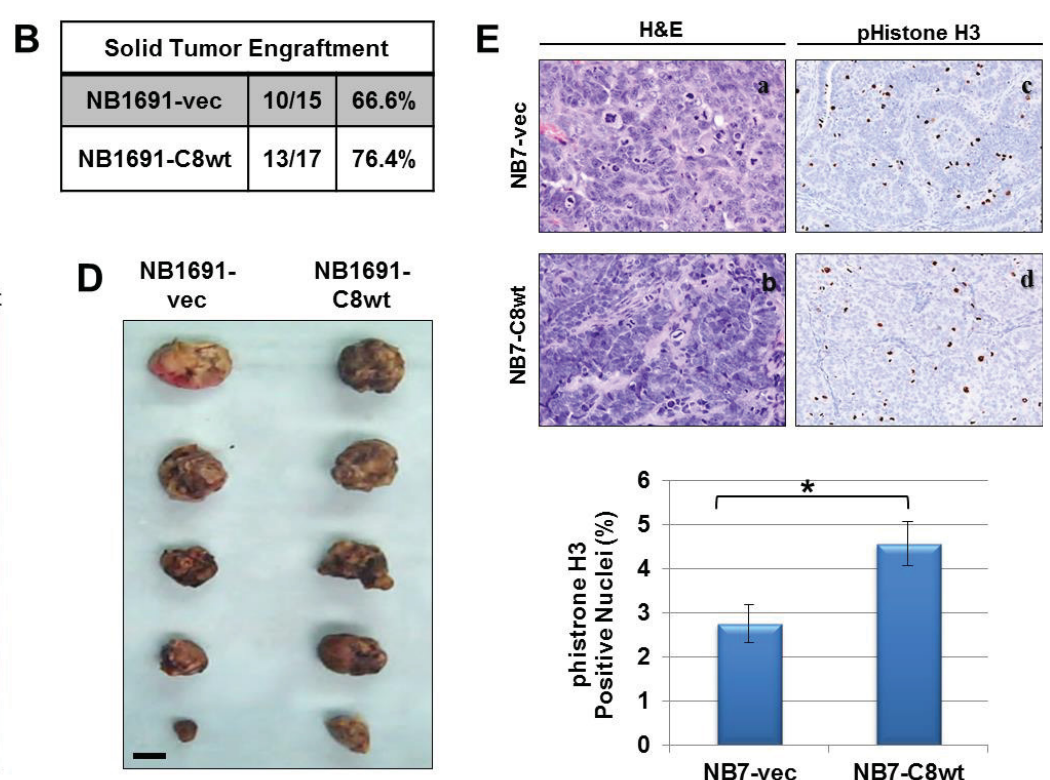
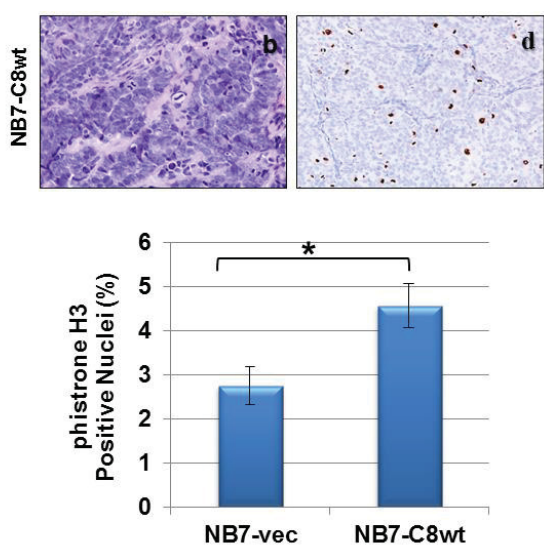

Figure 2-2. Increased engraftment in caspase-8 positive NB7 cells. (A, B) Following either six weeks or ten weeks, enrolled mice were euthanized and analyzed visually for solid tumors. All mice that were maintained in the study were previously determined by bioluminescence to have had successful injections at the one and two week time point. (C, D) Image of five randomly selected tumors from either NB7 parental cell lines, NB7 or NB1691 cells, expressing either vector (vec) only or casp8 wild-type (C8wt) tumor bearing mice. Scale bar, $1 \mathrm{~cm}$. (E) Xenografts of NB7 cells deficient for caspase-8 (a, c) or reconstituted with wild-type $(\mathbf{b}, \mathbf{d})$ were transplanted into the para-adrenal region of nude mice and hematoxylin and eosin stained $(\mathbf{a}, \mathbf{b})$ or stained for phospho-Histone H3 (c, d). Quantification of positive phospho-Histone H3 staining from five representative tumors $(*, \mathrm{P}<0.05)$. 
tumors. Histological examination of NB7 derived tumors for H\&E, CD31, cleaved caspase-3, and GFP, showed little difference between vector control and casp8wt tumors (Figure 2-2E). Staining for phospho-Histone H3, a mitotic cell indicator, showed that casp8 moderately enhanced tumor cell proliferation $(\mathrm{P}<0.05)$. Phosphorylation of histone $\mathrm{H} 3$ promotes DNA condensation, a key step during mitosis.

Immunohistochemistry of similar markers in tumors derived from the NB1691 tumor types showed no significant differences.

\section{Loss of caspase-8 affects tumor engraftment}

Our orthotopic xenograft model demonstrated that casp8 promotes primary tumor engraftment and growth. To further validate these findings, we used a common metastasis xenograft mouse model whereby cells are injected via tail vein [166]. Metastasis of cells from the primary tumor involves invasion into the surrounding environment, intravasation, extravasation from the blood vessel, and colonization or engraftment of the tumor at new sites. The tail vein model allowed us to investigate the possible role of casp8 during the latter stages of metastasis, extravasation and colonization of solid tumors. Therefore, we injected NB7 cells with and without casp8 via tail vein into severe combined immunodeficiency (SCID)/CB17 female mice. Mice were euthanized nine to ten weeks after injection. Mouse tumors derived from the casp8deficient vector only NB7 cells arising in the adrenal medulla and ovaries were microscopic in size $(<1 \mathrm{~mm})$ in comparison to tumors derived from casp8 positive cells $(>1 \mathrm{~mm})$. Full necropsy on all mice validated NB colonization. Infiltrated tumor cells were identified by H\&E and immunohistochemistry (IHC) for the neuronal markers synaptophysin and chromogranin A. Notably, casp8 expression not only markedly enhanced tumor establishment in the adrenal medulla, but also in the ovaries, lymph nodes, kidneys, liver, lungs, peri-renal fat, and bone marrow, all of which showed no infiltration of casp8 deficient NB7 cells. These results in conjunction with our orthotopic studies strongly suggest a positive role for casp8 in primary tumor establishment and growth in low tumorigenic cells (Table 2-1).

Table 2-1. Loss of caspase-8 affects tumor engraftment.

\begin{tabular}{lcc}
${ } }$ & NB7-vec & NB7-C8wt \\
\cline { 2 - 2 } & $0 / 57^{*}$ & $21 / 33$ \\
Ovary & $0 / 57^{* *}$ & $29 / 33$ \\
Lymph node & $0 / 57$ & $17 / 33$ \\
Kidney & $0 / 57$ & $14 / 33$ \\
Peri-renal fat & $0 / 57$ & $13 / 33$ \\
Liver & $0 / 57$ & $13 / 33$ \\
Lung & $0 / 57$ & $4 / 33$ \\
Bone marrow & $0 / 57$ & $1 / 33$ \\
\hline
\end{tabular}

Notes: microscopic tumors *37\% (21/57), **21\%(12/57) 


\section{Tumorigenesis does not require the proteolytic activity of caspase-8}

Caspase- 8 is predominantly known for its canonical role as a catalytic enzyme which mediates cell death by the cleavage of various substrates upon activation; however, recent studies indicate that casp8 controls several additional functions independent of its catalytic property, such as the activation of ERK1/2 by epidermal growth factor (EGF) and promoting NF- $\mathrm{KB}$ activation [87]. In order to determine whether the catalytic role of casp8 is required in NB tumorigenesis, we rendered casp8 enzymatically inactive by site directed mutagenesis of cysteine 360 to alanine (C360A). NB7 cells were stably transfected with the $\mathrm{C} 360 \mathrm{~A}$ casp 8 mutant and protein expression levels determined by western blot analysis (Figure 2-3A). To confirm that the point-mutation of C360A rendered casp8 catalytically inactive, we exposed our NB7 vector control, casp8wt, and casp8 C360A mutant cells to TRAIL [50 ng/mL] and MG132 [1 $\mu \mathrm{M}]$ for 24 hours and then blotted for casp8 cleavage and downstream cleavage events of active casp8 (Figure 2-3B). MG132 is a potent proteasome inhibitor that sensitizes NB7 cells to death receptor-mediated apoptosis. We demonstrated that casp8 C360A activity was significantly compromised as previously reported [73, 76, 167]. Investigation as to whether the catalytic activity was required for xenograft engraftment and tumor growth, NB7 casp8 C360A cells were orthotopically injected into CD1 nude mice. Mice were analyzed as previously described. Briefly, mice were monitored weekly for bioluminescence and radiance measured. A two log increase in tumor bioluminescence was predetermined as tumor burden, as previously described. Mice were sacrificed at tumor burden or at the end of the ten week study. Bioluminescent imaging revealed that tumors derived from the NB7 casp8 C360A cell line grew moderately faster by seven weeks $(\mathrm{P} \leq 0.05)$, as compared to the NB7 casp8wt xenografts (Figure 2-3C). For comparison, NB7 vector control and NB7 casp8wt data is presented from Figure 2-1. Overall, this data suggests that the catalytic activity of casp8 may hinder tumor growth. This conclusion supports previous research by others demonstrating that the catalytic activity is not required for cell survival $[87,91,92]$. Survival of mice transplanted with NB cells expressing casp8wt and catalytically inactive casp8 are essentially equivalent at 10 weeks $(\sim 15 \%)$; however, mice bearing casp8 C360A NB tumors have a higher mortality rate compared to those with NB casp8wt tumors starting at seven weeks (Figure 2-3D). By ten weeks, most mice enrolled in the NB7-casp8 C360A study had been euthanized due to tumor burden. Figure 2-3E is a representative bioluminescent image of mice bearing tumors deriving from NB7 vector control, casp8wt, or casp8 C360A cell lines at seven weeks post injection set to a scale of $1 \times 10^{8}$ to $5 \times 10^{9}$ radiance (Figure 2-3E). These data demonstrate that the catalytic activity of casp8 suppresses tumor growth and promotes cell survival. Furthermore, these findings support the hypothesis that the regulation of the canonical proteolytic and apoptotic function of casp8 may foster a more noncanonical prosurvival activity.

\section{Catalytically inactivated caspase-8 did not enhance tumor engraftment}

Ten weeks after orthotopic injection, mice enrolled in the NB7 casp8 C360A study not having reaching tumor burden were euthanized and visually inspected for the 

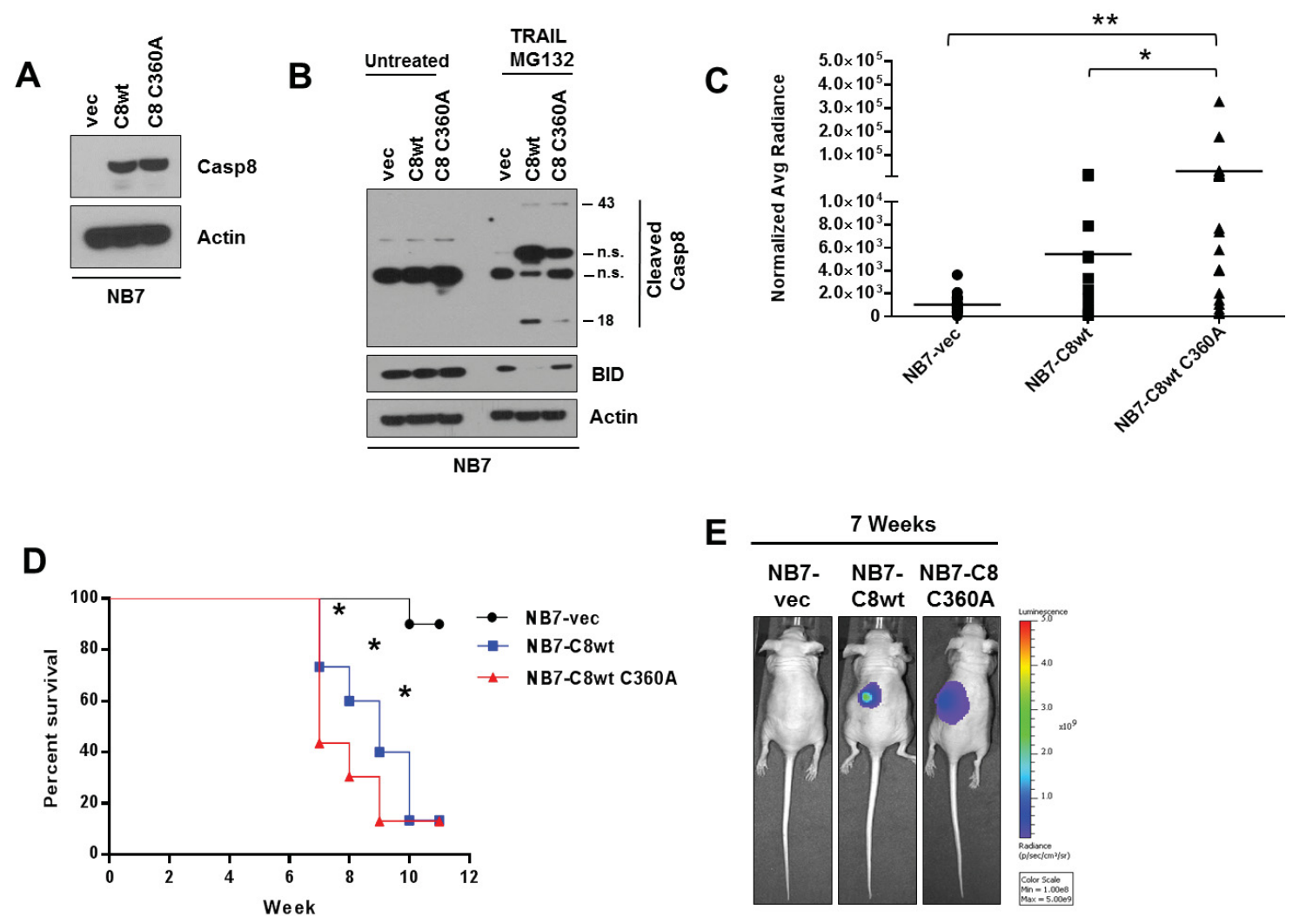

Figure 2-3. Catalytic activity of casp8 suppressed tumor growth.

(A) Expression of casp8wt (C8wt) and the catalytically inactive casp8 (C360A) in NB7 cells. (B) NB7 cells expressing both casp8wt and the mutant caspase-8 were treated with TRAIL [50 ng/mL] and MG132 [1 $\mu \mathrm{M}]$ for 24 hours and analyzed for casp8 activation and casp8-mediated signaling (n.s., not specific) (C) NB7 cells expressing vector (vec) only, C8wt, or the C360A mutant casp8, were orthotopically injected into the paraadrenal region of nude mice. Graph represents normalized bioluminescence at the seven week time point (*, $\mathrm{P} \leq 0.05, * * \mathrm{P} \leq 0.03$ ). (D) Survival plot of tumor bearing mice. Mice demonstrating a two log increase in bioluminescence were removed from the study (*, P $<0.0001$ ). (E) Representative bioluminescent images of tumor bearing mice. NB7-vec and C8wt data was shown previously and is only shown for comparative purposes. Error bars, \pm s.e.m. 
presence of a solid tumor mass. Engraftment rates were calculated based on the total of mice in the study and the number of mice presenting with solid tumors after ten weeks. These results were unbiased in regard to tumor size. NB7 casp8 C360A cells demonstrated a 96\% engraftment rate which was identical to NB7 casp8wt cells (Figure 2-4A). Interestingly, the inactivation of casp8 enhances tumor growth; though has no obvious effect on tumor engraftment. These data imply that casp8 may modulate various signaling pathways during tumorigenesis based on its catalytic activity affecting proliferation and colonization. As previously stated, all mice, prior to enrollment into the study were analyzed by bioluminescence weekly and luminescent signal was required to be maintained for at least two weeks post injection. Accurate tumor volume was difficult to obtain due to the involvement and engulfment of regional organs. Therefore, we randomly selected five tumors derived from the indicated cell lines as a visual representation of tumors extracted from mice (Figure 2-4B). Visually we noticed no significant size differences between NB7 casp8wt and NB7 casp8 C360A tumor masses. Analysis of phosphorylated Histone $\mathrm{H} 3$ in positive cells showed that casp8 C360A tumors were similar in proliferation compared to casp8wt expressing tumors and more than NB7 vector control tumors (Figure 2-4C).

\section{The protumorigenic function of caspase-8 is dependent on the prodomain}

Caspase- 8 contains multiple domains that may have unique and specific functions independent of its proapoptotic function. The DED domain of casp8 is crucial for the activation of ERK1/2 by epidermal growth factor (EGF) independent of the proteolytic activity of casp8 [87]. The DED domain of casp8 also has been shown to induce NF- $\kappa B$ activation and bind to perinuclear microtubules during paclitaxel-mediated apoptosis and during differentiation of skin and myeloid cells $[92,111,112]$. To determine the contribution of the DED in tumorigenesis, truncated casp8 (deleted amino acids 210-479) comprising the large and small subunits of the catalytic domain (CD) was stably expressed in NB7 cells (NB7-casp8 CD) (Figure 2-5A). Protein expression of casp8 CD was determined by western blot analysis (Figure 2-5B). NB7 casp8 CD cells were then orthotopically injected into nude mice following the procedures as described in the Materials and Methods section (Chapter 2). Mice were analyzed weekly for bioluminescence, mice lacking or losing luminescent signal before the two week time point were removed from the study. At seven weeks post injection, NB7 casp8 CD derived xenografts expressed bioluminescence at a statistically higher level compared to NB7 vector control xenografts (Figure 2-5C). At seven weeks there was no statistically significant difference between NB7 casp8wt and NB7 casp8 CD xenografts. Surprisingly, we noticed at time points eight, nine, and ten weeks that the luminescent signal for NB7 casp8 CD cells remained relatively consistent showing little to no tumor growth. We then compared the overall survival rates of NB7 casp8 CD cells to NB7 vector control and casp8wt xenografts (Figure 2-5D). NB7 casp8 CD xenografts demonstrated a slightly lower survival rate from the control NB7 vector control xenografts. An increase in overall survival supports our bioluminescence data demonstrating that NB7 casp8 CD xenografts do not grow as rapidly as NB7 casp8wt xenografts. NB7 casp8 CD cells demonstrated a 72\% survival rate while NB7 vector 


\begin{tabular}{c|c|c|c|}
\hline \multirow{3}{*}{ Solid Tumor Engraftment } \\
\cline { 2 - 3 } & NB7-vec & $19 / 36$ & $52.7 \%$ \\
\hline NB7-C8wt & $21 / 22$ & $95.5 \%$ \\
\hline $\begin{array}{c}\text { NB7-C8 } \\
\text { C360A }\end{array}$ & $23 / 24$ & $95.8 \%$ \\
\hline
\end{tabular}
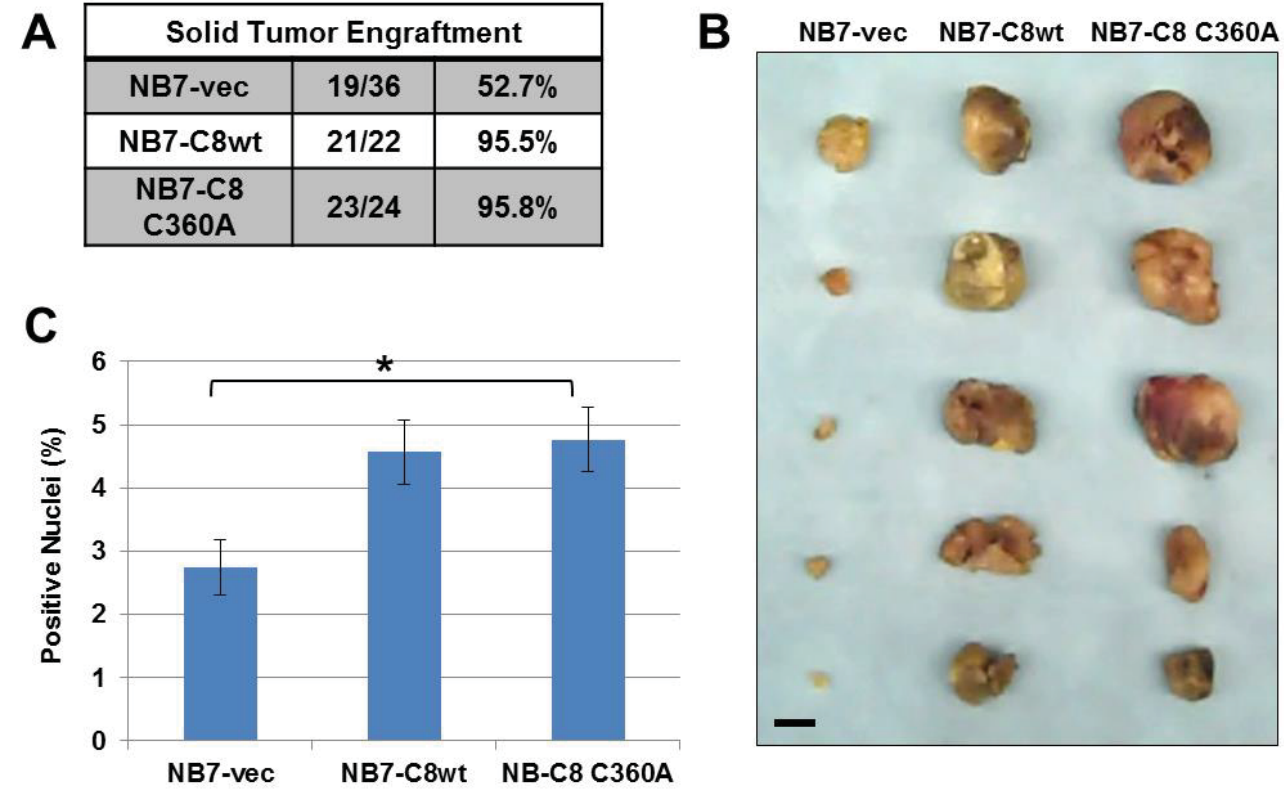

Figure 2-4. Inactivated of caspase-8 did not enhance tumor engraftment.

(A) Images of tumors randomly selected from the indicated NB7 cell line. Scale bar, 1 $\mathrm{cm}$. (B) After 10 weeks, mice enrolled in the study which had not reach tumor burden were euthanized and analyzed visually for solid tumor engraftment. NB7-vec and C8wt data was previously shown. (C) Quantification of positive phospho-Histone H3 staining from five representative tumors $(*, \mathrm{P} \leq 0.05)$. 


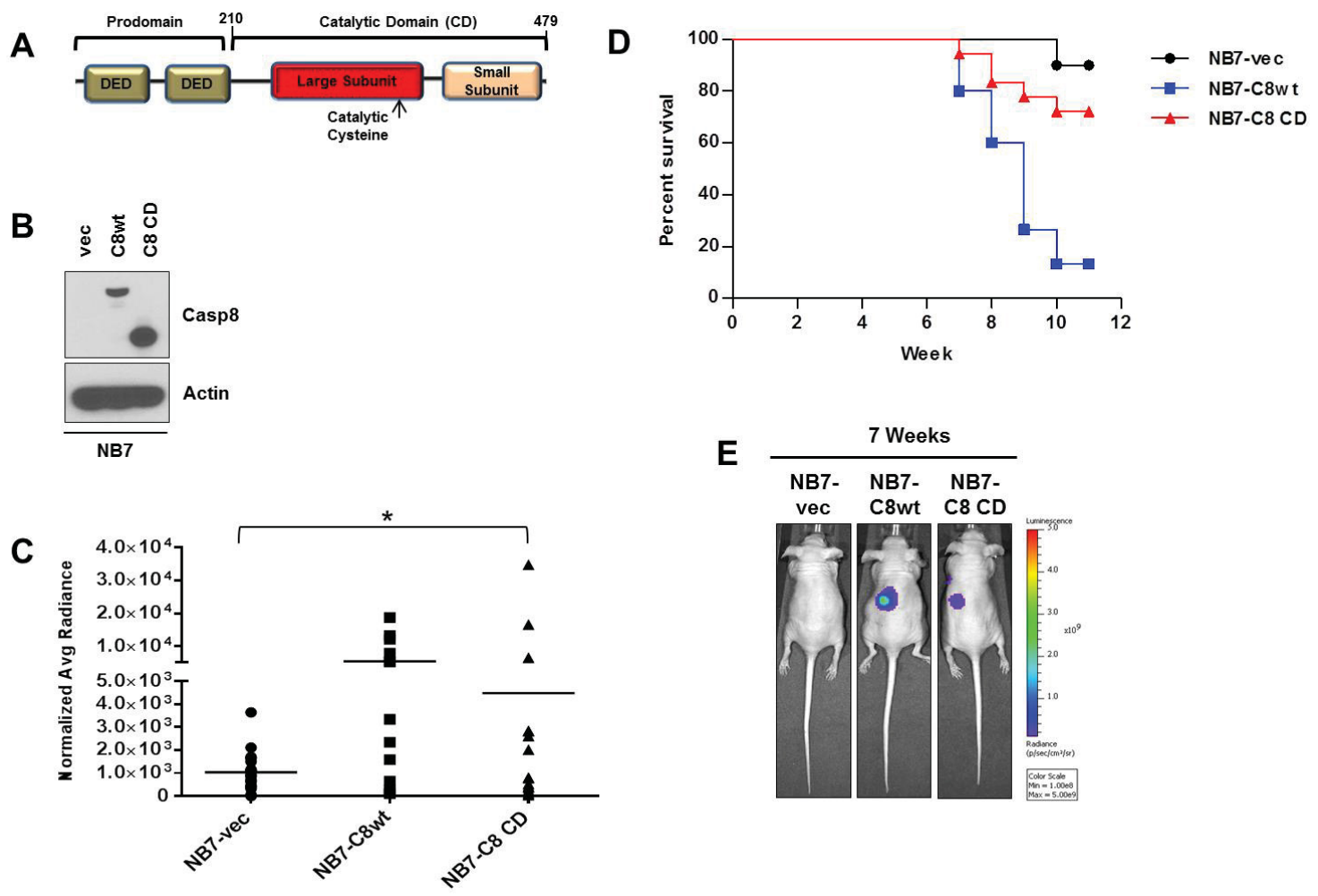

Figure 2-5. The prodomain is essential for caspase-8 tumorgenicity.

(A) A cartoon representation of casp8 depicting the truncated construct of casp8 comprising of catalytic domain from residue 210-479. (B) Protein expression levels of the truncated form of casp8 CD (C8 CD) compared to casp8wt (C8wt) NB7 cells. (C) NB7 cells expressing vector (vec) only, casp8wt, or truncated casp8 were orthotopically injected into the para-adrenal region of nude mice. Graph represents normalized bioluminescence at the seven week time point $(*, P<0.005)$. (D) Survival plot of tumor bearing mice. Mice with tumors exceeding a two log increase in bioluminescence were removed from study. (E) Representative bioluminescent images of tumor bearing mice. NB7-vec and C8 data was previously shown. Error bars, \pm s.e.m. 
control and casp8wt cells had overall survival of $90 \%$ and $13 \%$, respectively.

Figure 2-5E is a representative bioluminescent image of NB7 vector control, casp8wt, and casp8 CD cells at time point seven weeks. Radiance scale was set with a minimum of $1 \times 10^{8}$ to a maximum of $5 \times 10^{9}$ radiance. All NB7 vector only and NB7 casp8wt data was previously detailed in Figure 2-1 and is shown here for comparative purposes.

\section{Lack of the N-terminal prodomain suppresses tumor growth}

To determine whether NB7 casp8 CD cells were able to establish solid tumors in the para-adrenal region, transplanted mice were euthanized at the ten week time point and subjected to detail pathological review. Notably, NB7 casp8 CD cells demonstrated a reduced tumor establishment rate (72\%) compared NB7 casp8wt cells $(95 \%)$ (Figure 2-6A). Furthermore, NB7 casp8 CD tumors were slightly larger than the NB7 vector control xenografts, but markedly smaller than NB7 casp8wt tumors. Quantitative analysis of tumor size was unattainable due to the difficulty of obtaining accurate measurements since tumors often involved other organs. Probing for phospho-Histone $\mathrm{H} 3$ positive cells showed that casp8 CD tumors were significantly less proliferative compared to casp8wt expressing tumors (Figure 2-6C). These findings are consistent with the prolonged survival rate of mice bearing NB7 casp8 CD xenografts compared to animals harboring NB7 casp8wt tumors (Figure 2-5D).

\section{Overall expression of caspase-8 is diminished in orthotopic xenografts}

Previous data suggests that primary tumor formation is unaffected by casp8 expression; however, in secondary metastatic tumors, casp8 expression is seemingly selected against [145]. We sought to determine if in our orthotopic xenograft model if casp8 expression is maintained. Therefore, we performed western blot analysis on tumors derived from NB7 cells expressing casp8 wt. NB7 vector only, and casp8 parental cell lines were used as our negative and positive control, respectively (Figure 2-7A). Each well contains $30 \mu \mathrm{g}$ of total protein lysate. To lessen the possibility of contaminating mouse cells in our lysate and affecting total human protein concentration, we quantified casp 8 expression levels by densitometric analysis normalized to the individual GFP expression and compared to casp8/GFP parental cell line. Densitometric analysis revealed that only $20 \%$ of NB7 casp8wt expressing cells maintained relatively similar levels of casp8 (Figure 2-7B). RT-PCR analysis demonstrated that approximately $25 \%$ of NB7 casp8wt tumors suppressed casp8 at the transcript level while the remaining $75 \%$ seemingly regulated casp 8 at either the translational or posttranslational level (Figure 2-7C). Furthermore, casp8 expression in NB7 casp8 C360A tumors had a decrease in overall casp8 expression similar to casp8wt xenografts; however, NB7 casp8 CD maintained expression of the catalytic domain (Figure 2-8A). Caspase-8 lacking the DED domains essentially rendered casp8 enzymatically inactive and posed no known apoptotic threat; which may explain why the maintenance of casp8 CD expression in xenografts. These findings may suggest that casp8 may promote cell survival signaling during the initial or early stages of 


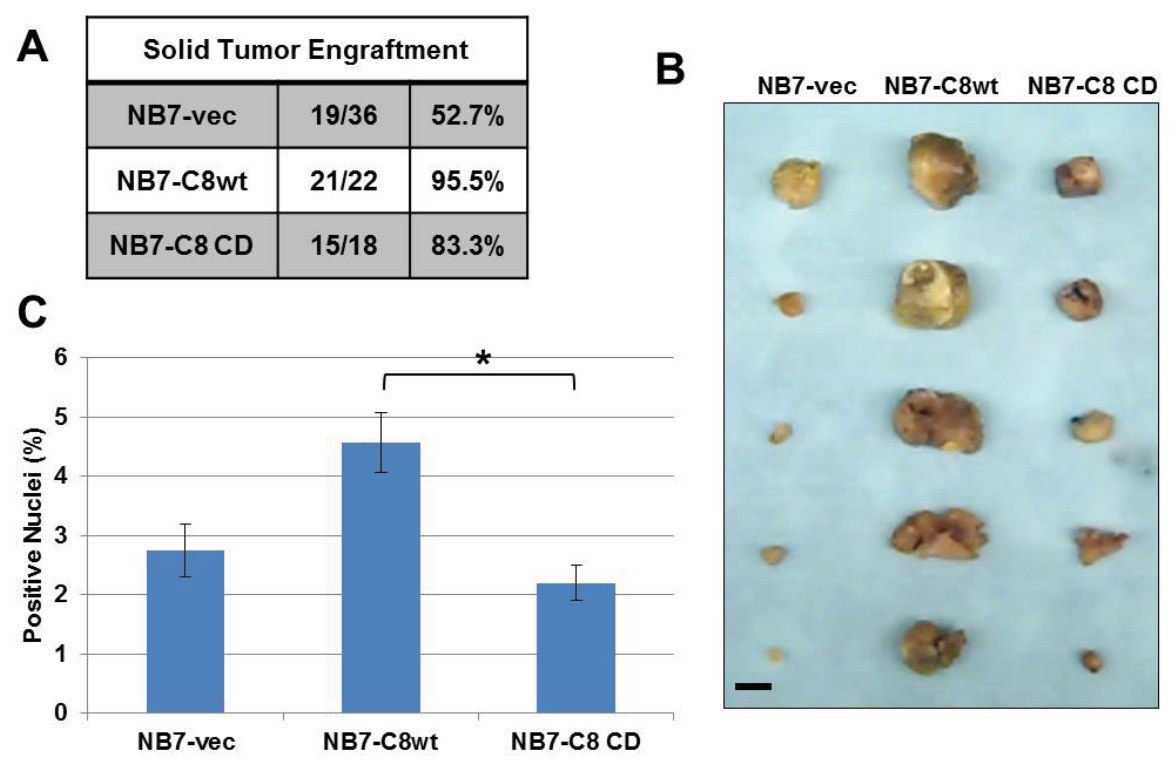

Figure 2-6. The N-terminal prodomain is required for tumor growth.

(A) Randomly selected orthotopic xenografts from the indicated NB7 cell lines. Scale bar, $1 \mathrm{~cm}$. (B) After ten weeks, mice were analyzed visually for solid tumor engraftment. Comparison of NB7 cells with and without casp8wt was shown previously. (C) Quantification of positive phospho-Histone $\mathrm{H} 3$ staining from five representative tumors $(*, \mathrm{P}<0.01)$. 


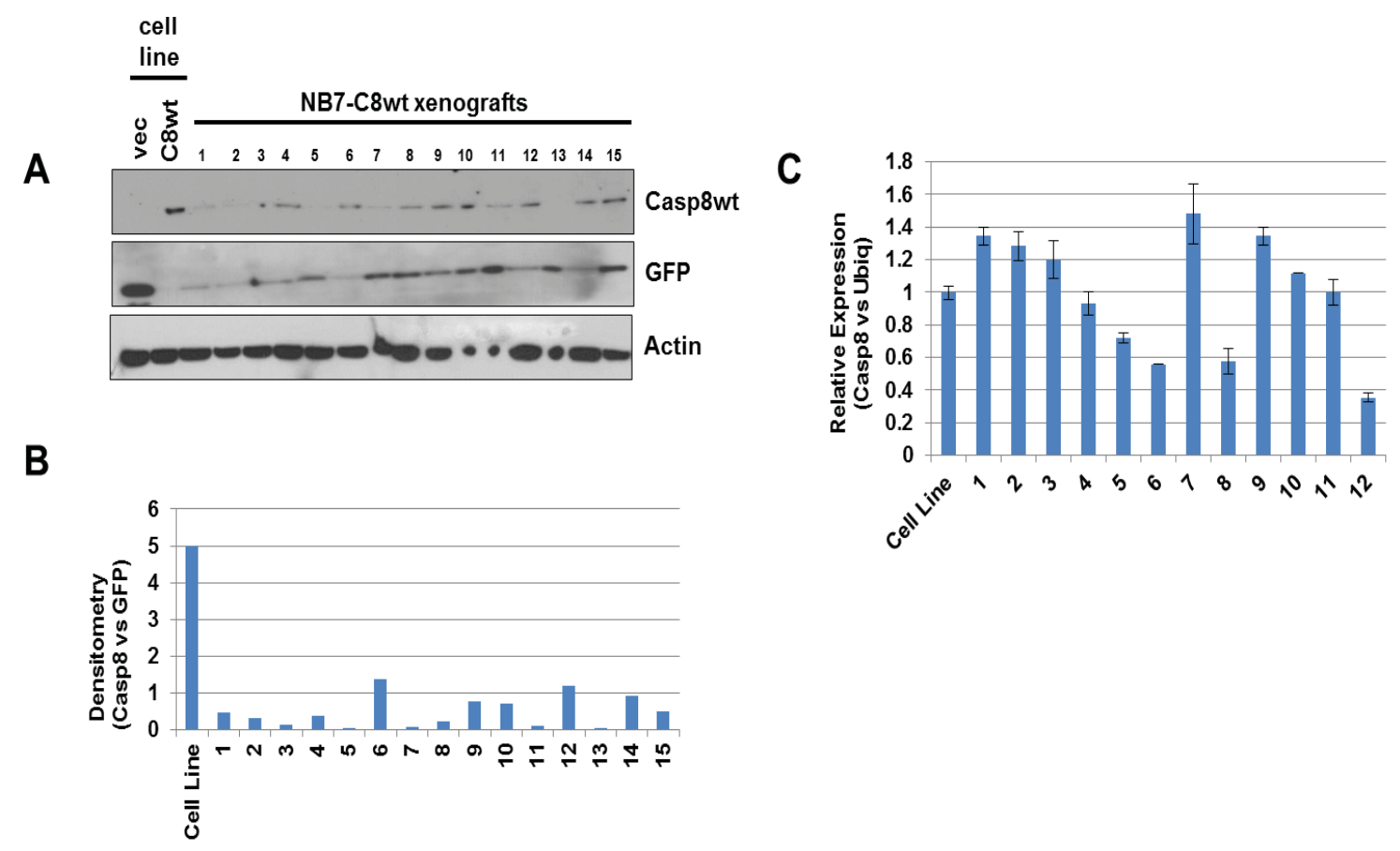

Figure 2-7. Caspase-8 is diminished in orthotopic xenografts.

(A) Caspase-8 expression in tumor xenografts derived from NB7 cells expressing casp8wt. Control lanes consist of parental cell lines reconstituted with either vector only or indicated casp 8 construct. (B) Caspase- 8 expression levels, as determined by western blot, were analyzed by densitometry for casp 8 expression and normalized to the representative GFP expression level and compared to control cell line. (C) Relative expression of casp8 mRNA normalized to ubiquitin. Xenograft numbers are the same for each experiment. Error bars, \pm s.d. 
tumorigenesis. NB1691 vector and casp8wt cells were also analyzed for casp8 expression revealing that casp8 is lost at three to six weeks post-injection (Figure 2-8B).

\section{Discussion}

Caspase- 8 is shown to be silenced in up to $70 \%$ of NB, $52 \%$ of medulloblastoma, and $79 \%$ of single cell lung carcinoma (SCLC) (79\%) [120, 122, 131, 143, 168, 169]. Loss of casp8 in tumors is shown to promote metastasis by suppressing IMD and decrease cell sensitivity to death promoting chemotherapeutic agents $[127,145,156]$. Taken together, these results support the notion that the loss of casp8 protects cells from apoptotic cell death and may promote tumorigenesis. Paradoxically casp 8 has been shown to have very opposing functions within the cell. For example, casp8 increased cell adhesion in tissue culture models and migration [88, 89, 145, 146, 149]. Caspase-8 expression is shown to be required for mouse embryogenesis for normal vasculature, heart, and neural tube formation [82]. Approximately 30-50\% of NB patient samples maintain casp8 expression. These data support on-going research demonstrating that casp8 not only has proapoptotic but prosurvival functions, independent of the enzymatic activity of casp8.

Most commonly in NB as well as other cancers, casp8 repression is a result of hypermethylation at $\mathrm{CpG}$ islands within the CASP8 promoter region $[117,121,123]$. Methylation of the promoter region regulates casp8 expression thus allowing, based on cellular conditions and signaling, for casp8 re-expression. During the wound healing process casp8 expression is silenced at sites proximal to the wound, though restored after the healing process demonstrating the possibility that casp8 is closely controlled and regulated [98]. This suggests that casp 8 may be expressed and silenced based on environmental signaling, stressors, and/or signaling events. Therefore, based on these data demonstrating that casp8 has prosurvival functions and may be regulated during tumorigenesis, we set out to test whether casp8 expression enhances NB tumorigenesis.

To investigate the potential biological role of casp8 in tumorigenesis, we developed a novel ultrasound guided orthotopic xenograft mouse model, which allowed us to inject our cells into the para-adrenal space of immunocompromised mice. We then selected two NB cell lines based on their tumorigenicity. Preliminary data suggested that the NB7 cell line is less tumorigenic in comparison to the NB1691 cell line. NB cell lines were stably transfected with casp8wt and validated for stable expression (Figure 2-1A). Endogenous casp8 is commonly expressed as a doublet represented by two different isoforms of casp 8 known as caspase-8/a and caspase- $8 / \mathrm{b}$. Biologically, no significant difference was observed between the two isoforms. Kinetics and proteinprotein interactions were similar between the isoforms. Caspase-8/a is slightly smaller due to the lack of approximately 15 amino acids from the $\mathrm{N}$-terminal region. Due to an extension of the N-terminal region from the DED domains, this lack of amino acids does not affect the DED domains of casp8 [164]. NB cell lines were reconstituted with the caspase- $8 / \mathrm{b}$ isoform. Densitometry analysis of exogenous casp8 protein level was compared to both isoforms as a whole, since both isoforms were functionally similar, and 


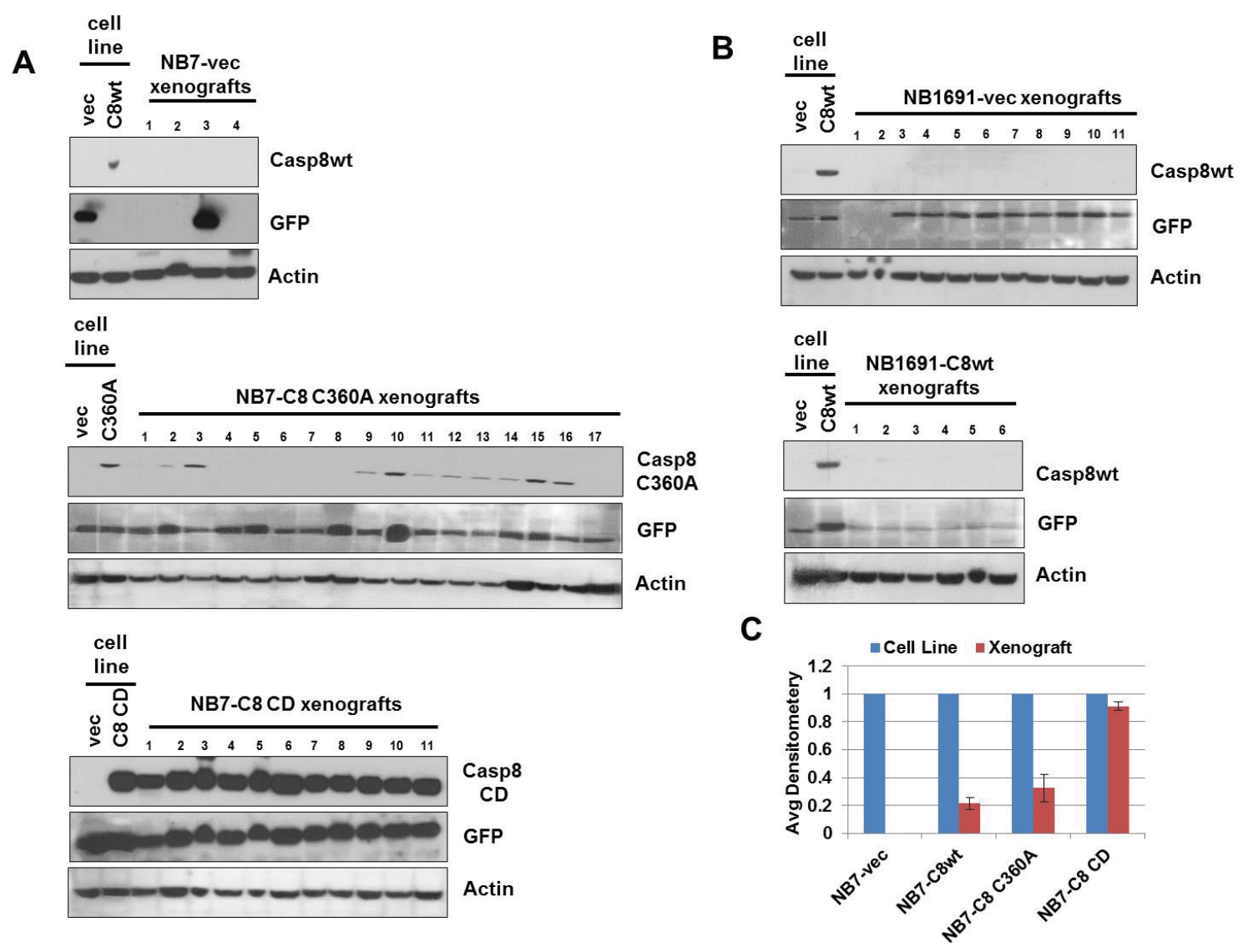

Figure 2-8. Overall expression of caspase-8 is diminished in orthotopic xenografts.

(A, B) Western blot analysis of casp8 expression in tumor xenografts derived from NB7/NB1691 vector control, casp8wt, and NB7 cells expressing either catalytically inactive casp8 (C360A) or truncated casp8 (catalytic domain only). Control lanes consist of parental cell lines reconstituted with either vector only or indicated casp8 construct. (C) Densitometry analysis of xenograft casp8 expression compared to control cell line Error bars, \pm s.e.m. 
confirmed that casp8 was reconstituted at relative physiological levels (data not shown). Surprisingly, NB7 tumorigenicity was significantly enhanced by casp8 expression, yet NB1691 with and without casp8 showed similar tumor growth. In previous studies, NB1691 cells were more tumorigenic than NB7 cells suggesting the possibility that genetically the NB1691 cells may have acquired additional mutations allowing for advanced tumor growth. Therefore, their requirement for casp8-mediated tumorigenicity was secondary and overall unnecessary. Based on this assumption, NB1691 cells are not informative to investigate tumorigenesis driven by casp8. Therefore, expanding our studies to involve other low tumorigenic casp8 deficient cell lines, such as NB10 and NB8, provide additional opportunities to challenge the role of casp8in promoting tumorigenesis. In addition, previous tumor growth studies demonstrate that NB10 and NB8 both have similar xenograft tumor growth rates as NB7 cells.

Previous studies performed in the chick chorioallantoic membrane (CAM) assay demonstrated that casp8 had no significant effect on tumor growth [145]. The CAM assay is extremely sensitive and often times overly sensitive, inducing an inflammatory and angiogenic response to an irritant $[170,171]$. The CAM assay has been extremely valuable for the analysis of angiogenesis and metastasis; though not commonly used for tumor growth studies and comparisons. Our orthotopic xenograft model implicating a role for casp8 in promoting tumorigenesis was previously tested and validated to effectively mimic the expression of key hallmarks of the TH-MYCN mouse model and NB patient tumor samples [165]. No such studies have been published for NB tumor growth in the CAM assay. NB7 cells with and without casp8, were seeded on the chorioallantoic membrane of fertilized chick eggs at a concentration of $5 \times 10^{6}$ cells per egg and tumors were harvested seven days later. In comparison, our orthotopic mouse model required $2 \times 10^{5}$ cells per mouse and up to ten weeks for tumor burden. The time frame of the orthotopic model closely mimics that of the transgenic TH-MYC mouse model and other xenograft mouse models using NB cells. Over seeding of NB cells may decrease selective advantages and obscure key cellular mechanisms when exposed to environmental pressures. This may have played a role in our inability to observe a difference in our NB1691 cells with and without casp8. Therefore, additional research is required to reassess NB1691cell number in our orthotopic mouse model.

The NB cell lines were reconstituted with two independent vectors expressing either casp8 and GFP or luciferase and YFP. To confirm that our vectors were not inadvertently impacting tumorigenicity, we orthotopically injected our parental NB7 cell lines lacking all vectors and constructs, into the para-adrenal region of nude mice. These cells were not manipulated in any form, which precluded the monitoring of tumor growth during the ten week study. After ten weeks, the tumors derived from the parental cell lines were visually compared to NB7 vector control and casp8wt expressing cells and noted to be significantly smaller than casp8wt, but very similar to vector control cells. Our data suggests that our vectors and our method of selecting for vector positive cells did not artificially select for a less tumorigenic cell colony or transform the cells

(Figure 2-2C). Furthermore, we injected our NB7 cells with and without casp8 into the flank of nude mice at the same cell concentration as our orthotopic model. Mouse flank tumors were analyzed weekly by bioluminescence for ten weeks. Tumor growth of the 
flank injected NB cells were similar to the para-adrenal xenografts, but did not reach tumor burden (Figure A-3C). These findings suggest that NB cells interacted similarly in both sites, but respond differently to environmental signaling and growth factors. Overall, casp8 enhanced NB tumorigenesis whether in the para-adrenal region or flank.

Visual analysis of our orthotopic xenografts suggested an increased level of vascularization in casp8 positive NB7 cells. Support for this observation derives from the casp8 knockout mouse which is embryonic lethal due to decreased vasculature as well as heart and neural tube defects [82]. An increase in angiogenesis may also explain why we observed an increase in tumor growth in NB cells expressing casp8wt. Histological examination of CD31 did not demonstrate a quantitative difference between NB7 cells with or without casp8. The conflict between our visual analysis and immunohistochemistry may be due to a small sampling size. Additional studies would include a larger sample number and include probing for additional endothelial and angiogenesis markers such as CD34, CD105, platelet endothelial cell adhesion molecule (PECAM-1), von Willebrand factor (vWf), and vascular endothelial growth factor (VEGF) will be necessary to address the potential role of NB in angiogenesis and consequently tumorigenesis.

To our knowledge, this is the first report demonstrating that casp8 may promote primary tumor formation and growth in NB cells. Confirmation of the protumorigenic potential of casp8 was performed by tail vein injection of NB7 vector and casp8wt cell lines. Analysis of tumorigenesis using the tail vein xenograft model specifically targets a cell's ability to extravasate from the blood vessel and colonize in a foreign environment. Our data demonstrates that casp8 positive cells significantly enhanced cellular extravasation, colonization within a foreign environment, and increased tumor formation in an increased number of foreign sites (Table 2-1). These results were confirmed by full necropsy, immunohistochemistry, and immunoblot analysis. This data supports data from our orthotopic xenograft model, as well as, that of previously published research demonstrating that casp8 enhances metastasis, in vitro [88, 91, 149]. Extravasation from the vasculature and migration into a foreign environment are stages of the metastatic process, both of which casp8 expression has been shown to enhance, in vitro.

Previous research demonstrated that casp8 enhances cell adhesion, invasion, and metastasis which supports our two xenograft models, orthotopic and tail vein, demonstrating a striking advantage to casp8 expression in NB tumorigenesis $[88,89,146$, 149]. In contrast, studies conducted in TH-MYC transgenic mice that that are conditionally deficient in casp 8 within the neural crest lineage demonstrate that casp8 limits NB tumorigenesis. Although loss of casp8 in this genetically engineered mouse model enhanced bone marrow metastasis, there was no difference in primary tumor formation with or without casp8 [152]. Unfortunately, this mouse model only represents approximately $30 \%$ of advanced-stage NB (stages 3-4) of which casp8 silencing is still controversial in this setting $[17,143,144]$. Furthermore, rarely is casp8 deleted in NB and is more commonly suppressed; thereby suggesting a mode of regulation and possible re-expression, as opposed to complete abolishment. Taken together with our xenograft data, casp8 may be essential not only in the initial primary tumor growth but during 
metastasis as well. In order to fully characterize the role of casp8 in NB tumorigenesis additional research exploiting both the conditional casp8 knockout mouse model as well as xenograft models will be required.

The prosurvival functions of casp8 may not be dependent on the catalytic activity or domain. For example, the DED domains of casp8 initiated ERK1/2 activation when exposed to EGF while studies showed that the catalytically inactive casp8 promoted NF$\kappa \mathrm{B}$ signaling $[87,93,94]$. Point-mutation of casp8 at a critical aspartic acid residue rendered casp 8 catalytically inactive and rescued the embryonically lethality of casp 8 knockout mice. This suggests that the developmental functions of casp8 may not be dependent on its enzymatic property. To investigate the functionality of the enzymatic property of casp8 in promoting NB tumorigenesis, we mutated casp8 at the catalytic cysteine 360; thereby rendering casp8 enzymatically inactive. Injection of our NB7 casp8 C360A cells into the para-adrenal region of immunocompromised mice demonstrated an increase in tumorigenesis as indicated by enhanced tumor growth by seven weeks and a decrease in overall survival from seven to nine weeks when compared to NB7 casp8wt derived tumors. Interestingly, independent of the catalytic activity of casp8, the overall survival of tumor bearing mice was $13 \%$ after ten weeks. Mice transplanted with cells expressing the catalytically inactive casp8 developed more pronounced tumors earlier indicated by more than a $20 \%$ decrease in survival at seven, eight, and nine weeks. Increasing our sample number and inclusion of earlier time points (five and six weeks) may more clearly define differences between the two casp8 constructs. Tumor engraftment, however, was not affected by the casp 8 catalytic activity. Interestingly, by ten weeks the overall survival of NB7 casp8wt cells was comparable to NB7 casp8 C360A tumors suggesting that the enzymatic activity of casp8 slowed the tumorigenicity of NB7 casp8wt cells. These results imply that the catalytic activity of casp8 hinders NB onset and that during NB tumorigenesis it is advantageous for NB cells to suppress the enzymatic activity of casp8. Furthermore, casp8wt enhances overall tumor growth and establishment yet inactivation of casp 8 promotes tumor growth and not establishment. This would suggest that casp 8 may have more than one signaling function based on its enzymatic activity during NB tumorigenesis; which affects both proliferation and colonization. In contrast, NB7 cells expressing only the catalytic domain of casp8 (NB7 casp8 CD) demonstrated an increased survival from 13\% in NB7 casp8wt derived tumors to $72 \%$. There was no statistical difference between NB7 vector and casp8 CD xenografts in overall survival; however, there was significant difference between casp8wt and the truncated mutant $(\mathrm{P}<0.001)$. Visual analysis of NB7 casp8 CD tumors clearly demonstrate a lack of tumor growth in comparison to NB7 casp8wt tumors and more closely resemble NB7 vector tumors. Truncation of casp8 maintained similar tumor engraftment rates as NB7 casp8wt tumors. This implies that during the various stages of tumorigenesis namely, colonization (i.e. engraftment) and primary tumor growth, casp8 may promote functions independent of its proteolytic activity and be domain specific. We propose that the catalytic domain is essential in the establishment of NB7 tumors demonstrating that binding to an adaptor protein, such as FADD or TRADD, is not required. Tumor growth may be enhanced by binding to the adaptor proteins, suggesting that two possible signaling pathways of casp8 may influence NB tumorigenesis. Furthermore, our data suggests that the enzymatic activity hinders tumor growth implying 
the possibility that the catalytic activity of casp8 during NB tumorigenesis is tightly controlled and/or regulated, possibly explaining why up to $70 \%$ of NB patient samples silence casp8 expression by methylation as opposed to deletion.

Previous research suggested that during tumorigenesis casp8 positive cells are selected against since casp8 deficient cells are protected against IMD [145]. Western blot analysis of our NB7 vector, casp8wt, casp8 C360A, and CD tumors revealed that overall casp8 expression is lost as indicated by densitometric analysis. Closer examination of casp8 expression on an individual tumor basis demonstrates that several tumors maintain basal levels of casp8 expression. Maintenance of casp8 expression may be tumor region specific or maintained for cell survival purposes. Since there is a lack of casp 8 antibodies that are useful for immunohistochemical analyses, we could not confirm if casp8 was expressed more prominently in some regions than others in the tumor. Analysis of casp8 mRNA expression suggests that casp 8 may be regulated by posttranslational regulatory methods, in this model. Similarly, we found no detectable expression of casp8 in our western blot analysis of tumors derived from NB1691 casp8wt cells. Additional research involving the regulation of casp 8 protein expression in NB at early and late stage tumorigenesis is warranted. This important issue may be addressed by establishing tumor explants or cell line cultures short term in vitro and casp8 expression monitored by pulsechase analysis with and without proteasomal inhibitors.

Overall, this data supports previous literature demonstrating that casp8 is a multifunctional protein independent of its proteolytic activity and domain. For the first time, these studies demonstrate a protumorigenic function of casp 8 as determined by an increased in tumor cell engraftment and proliferation in casp8 positive NB7 cells. These results were shown to be independent of the catalytic activity. Further research is required in order to elucidate the exact mechanisms of casp8 during NB tumorigenesis. We propose time course studies involving the extraction of primary tumor masses at specific stages post orthotopic injection into nude mice. These tumors would be monitored by ultrasound for tumor volume and by bioluminescence. Protein levels would be determined by western blot analysis and protein transcript levels by RT-PCR. Level of casp8 regulation in our xenograft models would include polysomal preps and pulse-chase experiments. Visual analysis of our tumors suggested that casp8 positive NB7 xenografts had a higher level of vasculature. To investigate the possibility of casp8 in NB angiogenesis, we suggest probing our xenografts with additional markers of angiogeneisis such as CD34, CD105, and VEGF.

The next critical aspect of characterizing the function of casp8 in tumorigenesis is to elucidate the mechanism and signaling pathways induced by casp8. Mechanistically, casp8 has been shown to have a higher affinity for FLIP heterodimerization as opposed to homodimerization with casp8. It has been suggested that casp8 homodimerization transduces apoptosis while heterodimerization mediates cell survival, due to FLIP inability to induce the intersubunit cleavage events of casp8. Caspase- 8 involvement with integrins has shown to promote ERK1/2 signaling and metastasis. Therefore, priority will be given in determining if casp8 heterodimerizes with FLIP or associates with integrins. To perform such experiments, casp8 will be tagged using His or FLAG in 
order to perform coimmunoprecipitation experiments from cells and xenografts. Controls will be conducted using bead only immunoprecipitations to determine non-specific binding.

\section{Materials and Methods}

\section{Cell lines}

Human neuroblastoma, NB7 and NB1691 cell lines have been previously described [17, 128]. Briefly, neuroblastoma cell lines were established by St. Jude Children's Research Hospital. All NB cell lines including were maintained in RPMI 1640 supplemented with 10\% FBS, 1\% glutamine and $0.1 \%$ gentamicin (Invitrogen, Grand Island, NY). All cells were maintained in a humidified atmosphere of $5 \% \mathrm{CO}^{2}$ at $37^{\circ} \mathrm{C}$. NB7 and NB1691 cell lines were reconstituted with casp8 using the pMSCV_IRES_GFP retroviral vector. Retrovirus supernatants were prepared as previously described $[17,172]$. Briefly, NB cells were cultured with viral supernatants plus polybrene $[4 \mu \mathrm{g} / \mathrm{ml}]$. Viral supernatant was replaced with fresh supernatant every 2 hrs for 6 total hours. Infections were allowed to occur for a total of 72 hours at $37^{\circ} \mathrm{C}$. After 72 hours, cells were sorted for GFP expression. Collected cells were cultured for two weeks prior to being sorted again for GFP expression. Luciferase expression in NB7 cells was accomplished using the pMSCV_Luc_IYFP construct packaged in the previously described retroviral transduction method. For NB1691 cells, 3x10 cells were electroporated at 200 volts with $30 \mu \mathrm{g}$ of the pMSCV-LucSh-zeocin construct and selected for zeocin resistance $[100 \mu \mathrm{g} / \mathrm{mL}]$.

\section{Reagents}

Mouse anti-casp8 (C15) was a gift from Dr. M. Peter [158]. Goat anti-actin (C11), was obtained from Santa Cruz Biotechnology, anti-rabbit GFP (A-11122) was from Invitrogen, anti-rabbit BID (2002) and cleaved casp8 (D391) were from Cell Signaling. rabbit anti-phospho-BID (S78) (A300-528A) from Bethyl Laboratories. All antibodies were used as recommended by manufacturer. Caspase- 8 mutations were developed by the QuikChange site-directed mutagenesis kit (Stratagene, La Jolla, CA) and manufacturer's recommendations followed. Mouse trueblot was purchased from Rockland Scientific (18-8817-33).

\section{Immunoblotting}

Cells were lysed in Nonidet P-40 lysis buffer (20 mM Tris (pH 7.5), $150 \mathrm{mM}$ $\mathrm{NaCl}, 1 \mathrm{mM}$ EDTA, and 0.5\% Nonidet P-40) plus $1 \mathrm{mM}$ PMSF, protease inhibitor cocktail (Roche, Mannheim, Germany) and phosphatase inhibitors ( $1 \mathrm{mM} \mathrm{NaF}, 1 \mathrm{mM}$ $\mathrm{Na}_{3} \mathrm{VO}_{4}$, and $1 \mathrm{mM}$ beta-glycerophosphate) for 5 minutes on ice followed by sonication. 
Lysates were centrifuged at 14,000 rpm for 10 minutes. Supernatants were removed and total protein was quantified using the Bradford assay (Bio-Rad, Hercules, CA). Proteins $(30 \mu \mathrm{g})$ were separated using SDS-PAGE analysis. Immunoblots were visualized using unlabeled primary antibodies and HRP-conjugated secondary antibodies. Protein expression was detected using enhanced chemiluminescence from GE Healthcare (Pittsburgh, PA). Quantification of band intensity was performed using the NIH ImageJ program.

Tumor samples were minced using a clean razor blade and suspended in ACK buffer $\left(0.15 \mathrm{M} \mathrm{NH}_{4} \mathrm{Cl}, 10 \mathrm{mM} \mathrm{KHCO} 3,0.1 \mathrm{mM}\right.$ EDTA) for 1 minute on ice to lyse RBC and repeated in needed. Tumors were subsequently washed with $\mathrm{PBS}$ and suspended in RIPA buffer (150 mM NaCl, $50 \mathrm{mM}$ Tris ( $\mathrm{pH} 8.0$ ), 0.5\% sodium deoxycholate, $0.1 \%$ SDS, 1\% NP-40) plus protease inhibitor (Roche, Mannehim, Germany). Tumor samples were maintained in RIPA buffer for 5 minutes on ice and sonicated until tumor dissolved. Protein concentration was determined using the Bradford assay (Bio-Rad, Hercules, CA). Proteins (30 ug) were separated using SDS-PAGE.

For immunoprecipitations, cells were lysed in Nonidet P-40 lysis solution 1 hour after exposure to either 10 Gy of irradiation using a ${ }^{137} \mathrm{Cs}$ source (Gammacell40extractor, MDS Norton) or $75 \mathrm{~J} / \mathrm{m}^{2}$ by a UV Strataliner 2400 (Stratagene). Caspase- 8 was pulled-down from $1 \mathrm{mg}$ of cell lysate using the C15 caspase- 8 antibody bound to protein $\mathrm{G}$ agarose beads (Pierce \# 20398) or cross-linked to $\mathrm{A} / \mathrm{G}$ agarose beads using a Pierce cross-linking kit (cat\#26147, Rockford, IL) and eluted from beads according to manufacture recommendation. Protein G agarose beads were washed in Nonidet P-40 lysis solution three times and suspended in SDS sampling buffer and boiled for 10 minutes.

\section{MTT}

Cells were cultured in 96 well plates under indicated conditions. At various time points, spent medium was removed and replaced with 20 ul phenol red-free RPMI 1640 containing $5 \% \mathrm{FBS}$ and $0.5 \mathrm{mg} / \mathrm{mL}$ MTT for 3 hours at $37^{\circ} \mathrm{C}$. Isopropanol (200ul) was added to dissolve the MTT formazan crystals. Absorbance was measured at $570 \mathrm{~nm}$ and background at $690 \mathrm{~nm}$.

\section{Orthotopic xenograft}

Ultrasound-guided orthotopic xenograft procedures were previously detailed [154]. Briefly, cells were suspended in Matrigel (BD worldwide, Cat\#354234, San Jose, $\mathrm{CA}$ ) at a concentration of $2 \times 10^{4}$ cells/ $\mu \mathrm{L}$ and kept on ice. Using a VisualSonics VEVO770 High Frequency Ultrasound system (VisualSonics, Toronto, Canada), a 22 gauge catheter was inserted into the para-adrenal space through the back muscle of anesthetized CD1 nude mice. A Hamilton syringe loaded with $10 \mu \mathrm{L}$ of cell suspension was guided steriotactically into the region between the kidney and adrenal gland and injected. For 
tumor growth analysis, mice were injected (intraperitoneal) with $3 \mathrm{mg} / \mathrm{mouse}$ of Firefly D-Luciferin (Caliper Life Sciences) and imaged using the IVIS® 200 imaging system. Bioluminescent images were analyzed using the Living Image 4.2 software (Caliper Life Sciences). Identical regions of interest (ROI) were used to determine the average radiance (photons $/ \mathrm{sec} / \mathrm{cm}^{2} / \mathrm{sr}$ ) of all images for all time points. For tail vein injections, cells were maintained in exponential growth and were suspended in serum free culture media at a concentration of $5 \times 10^{7}$ cells $/ \mathrm{mL}$. CB17/SCID mice were injected twice with $100 \mu \mathrm{l}$ of cell slurry with a 1 hour recovery time between injections. Mice were euthanized 9-10 weeks post injections and analyzed by full necropsy. Organs were analyzed for tumor cells using hematoxylin and eosin (H\&E), and for the neuronal markers synaptophysin, chromogranin A and PGP9.5. Digital calipers were used to measure tumor size.

\section{RT-PCR}

Total RNA was extracted from xenograft tumors with an RNA kit (Qiagen, Valencia, CA) according to manufacturers' recommendations. Complementary DNA was prepared with the iScript cDNA synthesis kit (Bio-Rad, Hercules, CA) from $1 \mu \mathrm{g}$ of RNA. IQ Syber green Supermix (Bio-Rad) and oligonucleotide primers Casp8-F (5'AAGCAAACCTCGGGGATACT), Casp8-R (5'-GGGGCTTGATCTCAAAATGA), GFP-F (5'-CACATGAAGCAGCACGACTTCT), GFP-R (5'-AACTCCAGCAGGACCATGTGAT), Ubiq-F (5'-ACCTGACCAGCAGCGTCTGATATT), and Ubiq-R (5'-

TCGCAGTTGTATTTCTGGGCAAGC) were used to perform a two-step real-time PCR assay. Samples were denatured at $95^{\circ} \mathrm{C}$ followed by an annealing and extension step at $62^{\circ} \mathrm{C}$. Relative expression was quantified by the $\Delta \mathrm{C}_{\mathrm{T}}$ method and normalized to either GFP or human ubiquitin. 


\section{CHAPTER 3. ROLE OF CASPASE-8 PHOSPHORYLATION IN NEUROBLASTOMA}

\section{Introduction}

Activation of casp8 mediated by adaptor protein binding (i.e. FADD or TRADD) provides significant measure of control and regulation over the extrinsic apoptotic pathway. Data demonstrates that binding partners such as inhibitor of apoptosis proteins, FLIP, and posttranslational modifications (i.e. ubiquitination, nitrosylation, and phosphorylation) add an increased measure of control over cell fate [107]. In regard to phosphorylation, both kinases and phosphatases control caspase activation and catalytic activity. These caspase-regulatory pathways limit or promote an apoptotic response based on changes in the environmental queues, stressors, metabolism, and growth factors. A recent review outlined the molecular events of caspases $(2,3,8$, and 9) phosphorylation and the biological significance of such modulation. Interestingly, the majority of phosphorylative events mediated by various kinases (i.e. DNA-PK, CaMKII, and Src) suppressed the catalytic activity of their caspase substrate [107].

As previously stated, the various functions of casp 8 may be regulated by localization, involvement with various macromolecular complexes, and/or posttranslational modifications $[90,99,100]$. Cursi et al. was the first to show that EGFmediated migration-induced casp8 phosphorylation by Src at Y380 [101]. This phosphorylation induced casp8 interactions with the p85 subunit of the phosphatidylinositol 3-kinase thereby increasing migration and adhesion. Later, Barbero et al. demonstrated that integrin ligation could also induce casp8 phosphorylation at Y380 and promote the localization of casp8 to the lamellae of migrating cells [102]. In addition, Cursi et al. further demonstrated that the phosphorylation event of Y380 suppressed the catalytic activity of casp 8 by approximately two-fold [101]. Caspase- 8 phosphorylation by cyclin-dependent kinase 1 (Cdk1) and ribosomal S6 kinase 2 (RSK2) at S387 and T263 respectively, led to a decrease in Fas-mediated apoptosis [103, 104]. p38MAPK phosphorylation of casp8 at S347 also suppressed the catalytic activities of casp8 [105]. Ataxia telangiectasia mutated (ATM) kinase, phosphorylated casp8 at S219 upon double strand DNA breaks, suggesting a possible crosstalk between DNA repair and apoptosis [106]. In cancer, several of these kinase are reported to be upregulated due to their prosurvival function. This increased kinase activity may contribute to altering the balance between the canonical function of casp8 to a more noncanonical or prosurvival function [107].

Cells are constantly exposed to natural DNA damaging signals that pose serious threats to genomic integrity $[173,174]$. Evolutionarily, cells have adapted elaborate signaling networks which regulate cell cycle progression, DNA damage repair, and/or cell death. DNA is subject to damage when exposed to reactive oxygen species (ROS), environmental chemicals, or exposure to ultraviolet radiation. If DNA damage is left unrepaired and allowed to be propagated to subsequent daughter cells, it would be detrimental to the organism as a whole. One particular form of DNA damage is double- 
strand break (DSB), in which both strands of DNA are severed. This DNA damage requires a specific DNA damage repair mechanism.

Ataxia telangiectasia (A-T) is a rare autosomal recessive disorder derived from a mutated gene product, ATM [175]. Clinically, A-T patients manifest with radiosensitivity, immune dysfunctions, telangiectasia, and an increased frequency of cancer [176]. ATM, a serine/threonine kinase, is a member of the phosphatidylinositol 3kinase-related kinase (PIKK) family and composed of a PI(3)K domain, a FAT (FRAPP/ATM/TRRAP), and a FATC domain [177]. Most commonly, mutations of ATM reside within the FATC domain [178]. A-T patients may also present with immune dysfunction, radiosensitivity, meiotic defects, and cancer [179].

DSBs recruit a heterotrimer complex known as the MRN complex (Mre11Rad50-Nbs1) which senses DNA damage and acts as the platform for ATM activation and DNA damage repair [180-182]. Binding of the MRN to DSB induces conformational changes of Rad50 promoting the tethering of the two breakage sites mediated by its flexible coiled coils and zinc-hook $[183,184]$. The Mre11 possesses nuclease activity while the C-terminal region of ATM interacts to Nbs1, driving ATM activation [185, 186]. ATM resides in the nucleus as an inactive homodimer. Upon DNA damage, ATM interacts with the MRN complex and undergoes autophosphorylation, which reduces ATM to an active phosphorylated monomer [187]. Activated ATM can phosphorylate several cell-cycle regulators including p53, p21, Nbs1, Chk1 (checkpoint kinase 1) and BRCA1 (breast cancer 1, early onset). In addition, ATM can signal for cell death via Chk2 (checkpoint kinase 2) and p53. DNA damage repair can be mediated through KRAB-associated protein 1 (KAP-1) and p53-binding protein 1 (53BP1) along with other repair proteins [188]. Overall, ATM can induce DNA damage repair, cell-cycle arrest, apoptosis, and has been known to play key roles in meiosis, homeostasis and cancer prevention [188].

Further studies on ATM activation revealed that ROS also led to ATM activation; however, signal transduction varies. Oxidative stress induces a disulfide bridge to form between the ATM homodimer. This disulfide bridge inhibits ATM to separate into active monomers [187]. The active ATM homodimer induces an antioxidant response, as opposed to a DNA repair response. The antioxidant response involves phosphorylation of heat-shock protein (HSP27); thereby leading to glucose-6-phosphate dehydrogenase (G6PDH) activation and an increase in nicotinamide adenine dinucleotide phosphateoxidase (NADPH). Increased levels of NADPH are linked to cell survival, and proliferation, making NADPH a potential target for cancer therapy [178, 188-191].

ATM is predominantly a nuclear protein; however, some studies report a cytoplasmic fraction of ATM. This suggests that ATM may have functions outside of DNA damage repair. ATM is shown to interact with the peroxisomes, modulate synaptic and vesicular function. In B cells, NF-кB stimulates the ATM-mediated phosphorylation of NEMO (NF- $\mathrm{KB}$ essential modulator), resulting in cytoplasmic relocation of the ATM/NEMO complex. This complex interacts with the IкB kinase leading to NF- $\mathrm{B}$ signaling [192-194]. In vivo studies of lymphocytes demonstrate that, upon DNA 
damage, ATM is essential for NF-KB mediated transcription [195]. ATM has also been linked to insulin signaling through the phosphorylation of eIF-4E-binding protein 1 (4EBP1), while ATM deficiency is linked to defects in mitochondrial function and mice deficient for ATM demonstrate an increased resistance to insulin and onset of atherosclerosis and metabolic syndrome [196-199]. In response to ROS, cytoplasmic ATM decreases the responsiveness of mTORC1 (mTOR complex 1) and autophagy promoting the activation of tuberous sclerosis complex 2 (TSC2) and hypoxia-induced factor (HIF-1).

ATM-mediated cell death upon DNA damage is thought to be dependent on the extent of DNA damage. Therefore, upon extensive DNA damage, ATM phosphorylates $\mathrm{p} 53$ at S15 leading to the optimal induction and activity of p53and induction of the intrinsic apoptotic pathway. As a transcription factor, active p53 mediates the transcription of various proapoptotic proteins, namely those of the Bcl-2 family, such as Bax, Puma, and Noxa [200]. At low concentrations of DSBs, ATM signals through NEMO, to induce NF- $\mathrm{KB}$ signaling and transduction of prosurvival genes such as cIAP and FLIP. Increased DNA damage induces an increase in TNF- $\alpha$ transcription by NF- $\mathrm{KB}$ signaling. TNF- $\alpha$ signaling through TNFR1 can transduce cell death mediated by casp8 activation and recruitment to the RIP1 complex [194]. Death receptor-mediated apoptosis induced by either TRAIL or FasL transduces the apoptotic signal through the activation of casp8, though the expression of inhibitor proteins such as FLIP can block casp8 mediated apoptosis. One study suggests that upon exposure to DNA damaging agents such as chemotherapeutic agents and/or IR induces DNA DSB and subsequent ATM activation. ATM activation enhances the ubiquitination and degradation of the long isoform of FLIP; thereby sensitizing cells to TRAIL and FasL mediated apoptosis [201]. BID, a well-known substrate of casp8, is phosphorylated by ATM, which causes BID localization and accumulation in the nucleus leading to cell cycle arrest in S-phase. A nonphosphorylatable BID mutant showed no S-phase accumulation and increased the cell sensitivity to DNA damaging agents, suggesting that ATM phosphorylation can modulate the proapoptotic function of BID to initiate cell survival [202].

Previous data suggests that casp 8 phosphorylation by various kinases suppresses catalytic activity and promotes cell survival. In this study, we hypothesized that the modulation of casp8 by phosphorylation promotes critical nonapoptotic functions during NB tumorigenesis. We aim to determine whether the phosphorylation event mediated by ATM upon DNA damage promotes casp8 phosphorylation and alters the balance between the canonical apoptotic function of casp8 and its noncanonical prosurvival role. We demonstrate that casp8 is phosphorylated at S219 in an ATMdependent manner and that phosphorylation suppresses the enzymatic function of casp8. In our model, we show that ATM phosphorylation has no significant effect on NB tumor cell engraftment or proliferation. Additional studies demonstrating the potential biological effect of casp8 phosphorylation at S219 are required. 


\section{Results}

\section{DNA damage promotes caspase-8 phosphorylation}

A large-scale proteomic study by Matsuoka et al., demonstrated that casp8 is phosphorylated at S219 post DNA damage in an ATM-dependent manner [106]. To validate these findings we studied $293 \mathrm{~T}$ cells and three NB cell lines expressing endogenous casp8 in response to 10 Gy of IR to induce DNA DSBs and subsequent ATM activation. Cells were allowed to recover for one hour prior to lysis and immunoprecipitation of casp8. Proteins were separated on an SDS-PAGE gel and blotted for phosphorylated casp8 (pCasp8) using the validated antibody recognizing the phosphorylated S78 epitope of BID (Figure A-1B). Caspase8 phosphorylation was detected in all four cell lines post DNA damage, as opposed to our non-irradiated cells and our bead-only immunoprecipitated (IP) negative control (indicated as C)

(Figure 3-1A). Immunoblot analysis also revealed that ATM was activated, thus suggesting the possibility that ATM upon activation may phosphorylate casp8. To determine whether phosphorylation of casp8 impacted apoptosis, casp8 S219 was mutated to either a nonphosphorylatable (S219A), or phospho-mimic (S219E) isoform. These constructs were stably transfected into NB7 and NB1691 cell lines. Expression levels were similar to casp8wt expressing NB7 and NB1691 cells (Figure 3-1B). We then exposed our NB7 and NB1691 cell lines containing the vector only, casp8wt, and our two phosphorylation mutants (S219A, S219E) to 10 Gy IR and probed for casp8 phosphorylation. Non-irradiated cells and beads-only served as negative controls and IP controls, respectively. In both NB cell lines exposed to IR and expressing the casp8wt revealed that a fraction of the total casp8 pulled down was phosphorylated; while no phosphorylation event was detected in the non-irradiated casp8wt control or irradiated casp8 mutants (Figure 3-1C). A kinase assay further confirmed casp8 phosphorylation upon ATM activation (Figure 3-1D). The casp8 doublet is formed from two Kozak sequences, as discussed previously [164]. Interestingly, the larger of the two casp8 constructs was phosphorylated at a higher frequency. Overall, these data suggest that casp8 is phosphorylated upon DNA DSB at S219 and further validates the specificity of our antibody.

\section{ATM-mediated phosphorylation of caspase-8}

Our previous results demonstrate that, upon irradiation, casp8 is phosphorylated as well as several other proteins. Therefore, we set out to validate whether ATM mediates the phosphorylation of casp8 at S219 upon irradiation. To test the role of ATM, we used a potent ATM inhibitor, Ku-55933, to block ATM activation upon DSB. We exposed our NB cell lines expressing either wild-type or the nonphosphorylatable mutant form of casp8 to $10 \mu \mathrm{M}$ of $\mathrm{Ku}-55933$ for 30 minutes prior to exposure to irradiation (Figure 3-2A). Our positive and negative controls consisted of casp8wt and casp8 S219A cells exposed to IR. NB7 and NB1691 lacked casp8 phosphorylation in casp8wt expressing cells when treated with IR plus inhibitor. Clearly, upon exposure to 

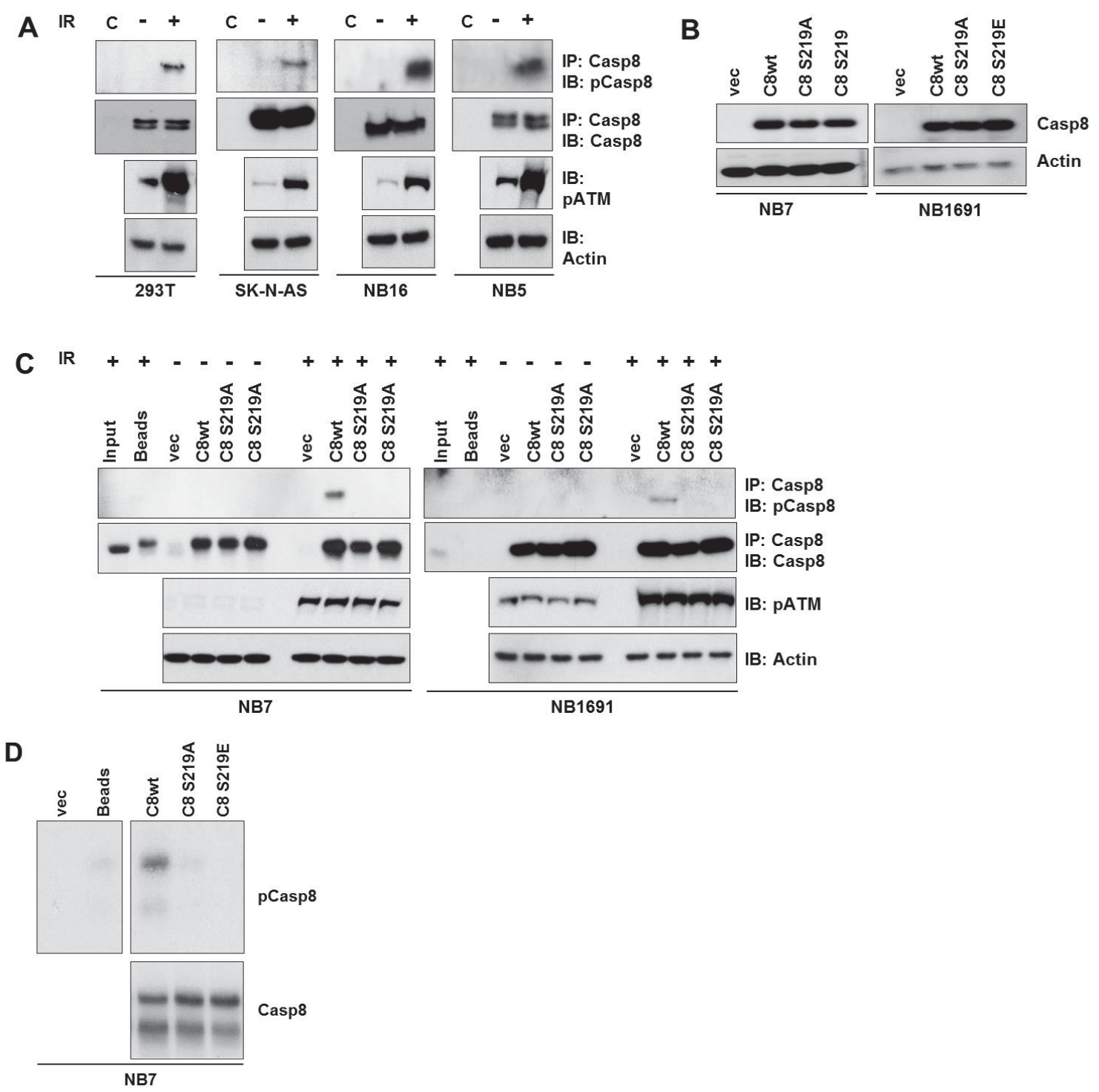

Figure 3-1. Caspase-8 phosphorylation at S219 post DNA damage.

(A) $293 \mathrm{~T}$ cells and three NB cell lines (SK-N-AS, NB16, NB5) were exposed to $10 \mathrm{~Gy}$ IR and analyzed for casp8 phosphorylation. Lanes indicated with a $\mathrm{C}$ mark control wells comprised of specific cell line exposed to IR and immunoprecipitated (IP) using beads only (without antibody). (B) Point mutagenesis of casp8 at S219 to either a nonphosphorylatable mutant (alanine) or a phospho-mimic mutation (glutamic acid). Expression levels of mutant casp8 in NB7 and NB1691 cell lines. (C) NB7 and NB1691 cell lines expressing the various constructs of casp8 (wt, S219A, S219E) or vector only exposed to $10 \mathrm{~Gy}$ IR and blotted for casp8 phosphorylation. (D) Kinase assay demonstrating in vitro phosphorylation of casp8. 


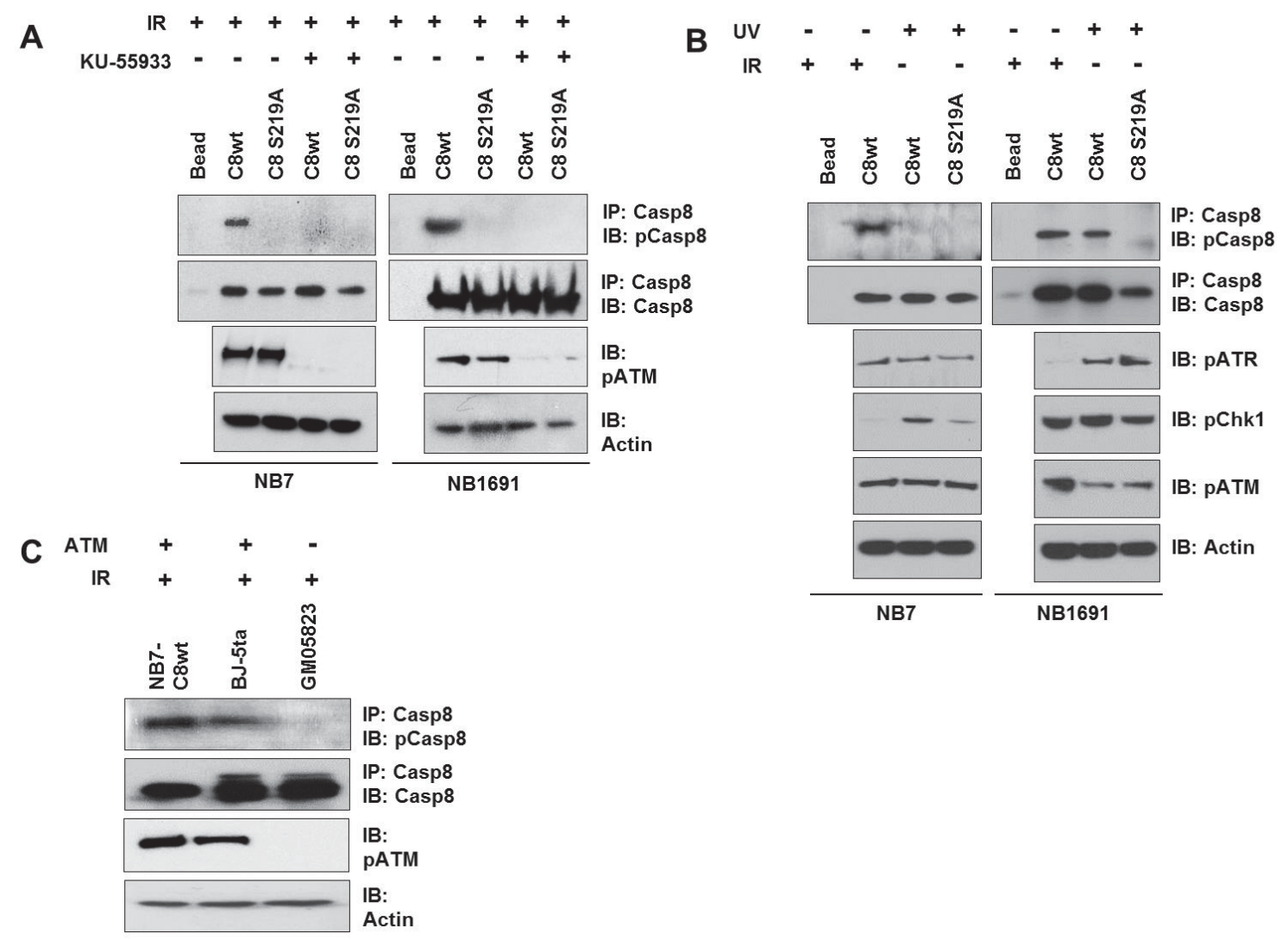

Figure 3-2. Caspase-8 phosphorylation at S219 is ATM dependent.

(A) NB7 and NB1691 cells lines expressing the indicated casp8 construct were exposed to $10 \mu \mathrm{M}$ of the ATM inhibitor, Ku-55933, 30 minutes prior to exposure to $10 \mathrm{~Gy}$ of IR. (B) Indicated NB cell lines expressing either vector control (vec), casp8wt, or the nonphosphorylatable mutant (S219A) were exposed to $75 \mathrm{~J} / \mathrm{m}^{2}$ of ultraviolet radiation and lysed one hour post exposure. (C) Human fibroblasts from an ataxia telangiectasia patient (GM05823), null for ATM expression, were exposed to $10 \mathrm{~Gy}$ and probed for phosphorylation of casp8 at S219. Normal human fibroblasts (BJ-5ta) and NB7-Casp8wt cells were used as positive controls. 
Ku-55933 ATM activation was effectively blocked and casp8 phosphorylation was not detected, suggesting that phosphorylation of casp8 may be ATM dependent. Work performed by Matsuoka et al and others demonstrated that ATM and ATR have similar phosphorylation sites and substrates [106]. One essential difference between these two kinases is the mode of activation. ATR is primarily activated upon single strand DNA breakage, but is also responsive to ultraviolet radiation (UV) induced DNA crosslinking between cytosine and thymine bases (pyrimidine dimers) [203]. Therefore, we exposed our cell lines expressing the indicated casp8 construct to UV at $75 \mathrm{~J} / \mathrm{m}^{2}$. Cells exposed to IR (10 Gy) were included as positive controls. Western blot analysis revealed that UV did not induce phosphorylation of casp8 in NB7 cells. By contrast, casp8 was preferentially phosphorylated in response to UV compared to IR (Figure 3-2B). To further challenge the role of ATM in casp8 phosphorylation we analyzed GM05823 human fibroblast cells derived from an ataxia telangiectasia (A-T) patient. These cells are null for ATM expression. Normal hTERT-immortalized human fibroblasts (BJ-5ta) and NB7 cells reconstituted with casp8wt were included as positive controls. All cells were exposed to $10 \mathrm{~Gy}$ of IR and analyzed for casp8 phosphorylation by western blot. As shown in Figure 3-2C, ATM defective cells lacked casp8 phosphorylation whereas casp8 was phosphorylated in BJ-5ta and NB7 casp8wt cells (Figure 3-2C). Taken together, these data support our hypothesis that casp8 phosphorylation is dependent on ATM activation.

\section{Caspase-8 phosphorylation is a cytoplasmic event}

ATM is primarily known as a nuclear protein. Some studies have demonstrated that ATM may become cytoplasmic and be involved in various cytoplasmic functions. A caveat of these studies is that cytoplasmic ATM has only been shown in cell lines and has yet to be shown in vivo. In contrast, casp8 is primarily cytoplasmic; though the Nterminal, prodomain, and active subunit of casp8, have been shown to localize to the nucleus under certain conditions $[108,111,112]$. Therefore, in order to further study ATM and phosphorylation of casp8 we determined if this post-translational modification event was cytoplasmic or nuclear. Control and IR-treated NB7 cells expressing the various constructs of casp8 were fractionated and blotted for casp8 and pATM. The purity of the nuclear and cytoplasmic fractions was determined by blotting for both $\beta$ lamin and $\alpha$-tubulin, respectively. Independent of DNA damage, casp 8 remained cytoplasmic. Long exposure of pATM revealed that a small fraction of ATM was located in the cytoplasmic fraction post DNA damage (Figure 3-3A). This suggests that upon DNA damage, pATM enters the cytoplasm and induces the phosphorylation of casp8. Support for this data comes from work by Biton et. al. demonstrating that pATM and NEMO translocate to the cytoplasm upon ATM activation [194]. Our data does not clearly demonstrate the localization of phosphorylated casp8; thus we immunoprecipitated our cytoplasmic and nuclear fractions with and without exposure to IR and show that phosphorylated casp 8 remains cytoplasmic (Figure 3-3B). Taken together, our data indicates that the phosphorylation event of casp 8 mediated by active ATM is a cytoplasmic event induced by a cytoplasmic fraction of ATM. 


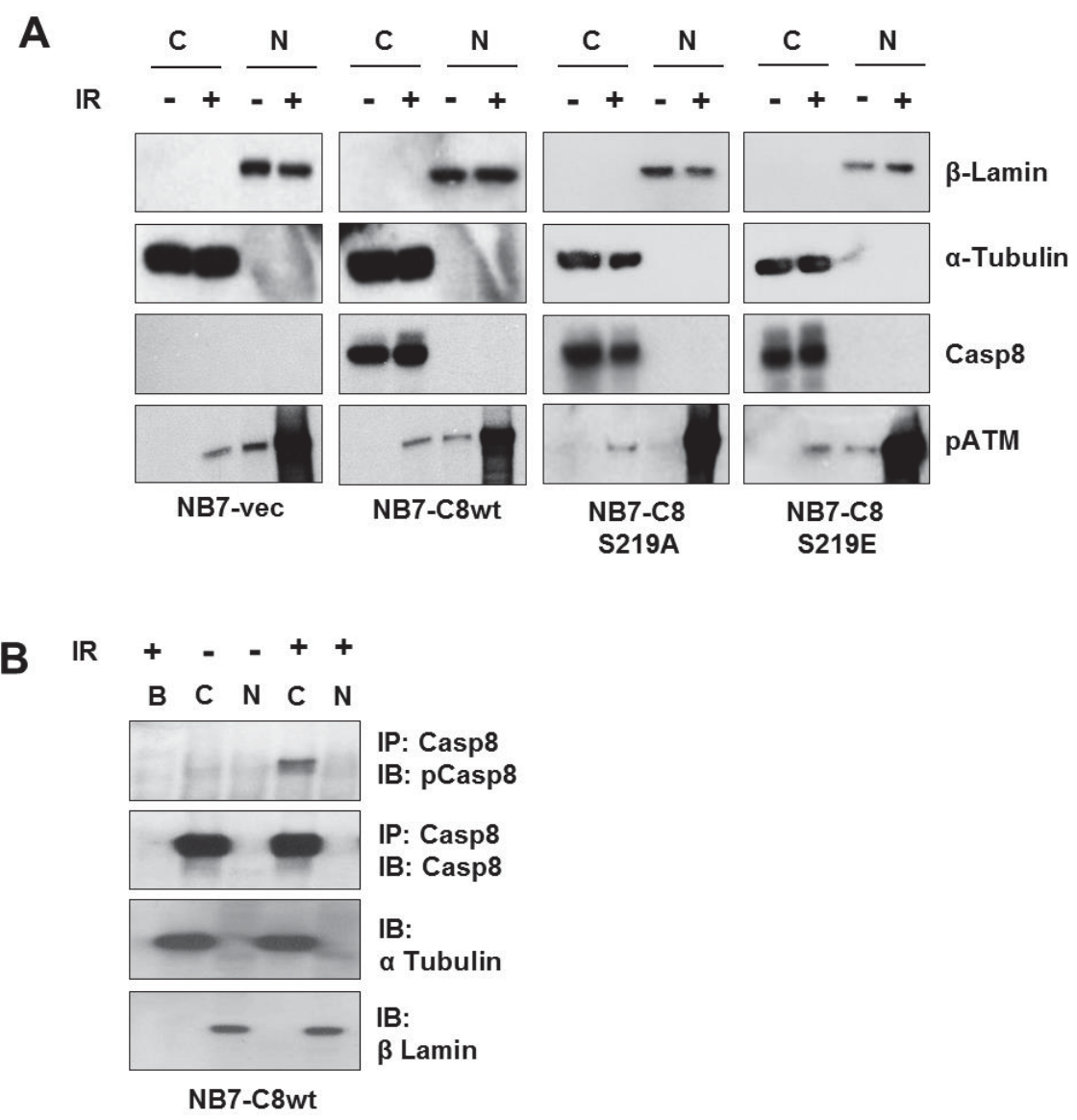

Figure 3-3. Phosphorylation of caspase-8 is a cytoplasmic event.

(A) Cell fractionation of NB7 cells deficient for casp8 or expressing either wt, S219A, or S219E mutations, were analyzed for cytoplasmic $(C)$ or nuclear $(\mathrm{N})$ localization of casp8 post DNA damage. (B) Using the previous cell fractionations, cytoplasmic and nuclear fractions were immunoprecipitated for casp 8 and detection of phosphorylated casp8 by western blot analysis. 


\section{Serine 219 of caspase-8 suppresses the catalytic activity}

A review of the literature reveals that there are eight phosphorylation sites on casp8. Phosphorylations of seven of these residues are mediated by known kinases and each suppress the apoptotic activity of casp 8 by approximately two-fold. We hypothesized that modulation of casp 8 would switch casp8 from its canonical apoptotic function to a noncanonical pro-survival protein. To investigate whether the catalytic activity of casp8 was affected due to phosphorylation, we exposed our NB7 cells expressing the various constructs of casp8, to TRAIL [50 ng/mL], MG132 [1 $\mu \mathrm{M}]$, and a proluminogenic substrate of casp8. This proluminogenic substrate upon cleavage by casp 8 emits a detectable luminescent signal that can be quantified $[103,104]$. Cells were harvested at various time points post exposure to TRAIL and MG132, as indicated (Figure 3-4A). At six hours NB7 casp8 S219E were significantly more resistant to apoptosis than casp8wt expressing cells $(\mathrm{P}<0.004)$. NB7 casp8 S219A cells were essentially as sensitive to the treatment as casp8wt expressing cells. Cell death was also quantified by measuring the impedance level of a biosensor. Cells are attached to gold electrodes at the bottom of 96-well plates. A small current signal $(1 \mu \mathrm{A})$ produces an electric field. Impedance of the electric field by cells can be quantified and measured in real-time. As indicated previously, cells were exposed to TRAIL and MG132 and subjected to real-time analysis of cellular impedance. Supporting our luminescent study, NB7 casp8 S219E cells had a higher baseline delta cell index, suggesting that these cells were less likely to undergo apoptosis as compared to NB7 casp8wt and casp8 S219A cells (Figure 3-4B). We propose that the phosphorylation at S219 may suppress the enzymatic activity of casp8 though not completely abolish it. Exposure to TRAIL and MG132 for extended periods demonstrated that NB7 C8 S219E cells were just as capable to undergo apoptosis as casp8wt (Figure A-2A). Overall, these data may suggest that ATM-mediated phosphorylation of casp8 may hinder casp8-mediated apoptosis under short exposure to apoptotic stimuli; thus allowing for ATM to drive DNA damage repair. Extended exposure to death-inducing stimuli (TRAIL) may overcome ATM-induced apoptotic hindrances and drive apoptotic cell death.

\section{D culture demonstrated increased colony formation}

NB7 and NB1691 cell lines were analyzed in vitro for changes in proliferation in response to wt and mutant casp8 expression. Interestingly, casp8 expression had no effect on proliferation in control and IR-treated 2D cell culture in either NB cell line (Figure 3-5A and B). In addition, the proliferation of cell expressing wt and mutant casp8 was similar when exposed to ROS and serum starved (data not shown). Our orthotopic xenograft mouse model displayed clear difference in cell proliferation with and without casp8 (Figure 2-1B), suggesting changes in environmental conditions may affect casp8 role in tumorigenesis. To address this issue in further detail, NB cells were suspended in a 3D collagen matrix and analyzed after three weeks in culture. Quantification of colony size demonstrated that within a 3D environment casp8wt and casp8 S219E expressing NB7 cells developed larger and more numerous cell colonies

(Figure 3-5B). These findings are consistent with the xenograft studies and suggest that 

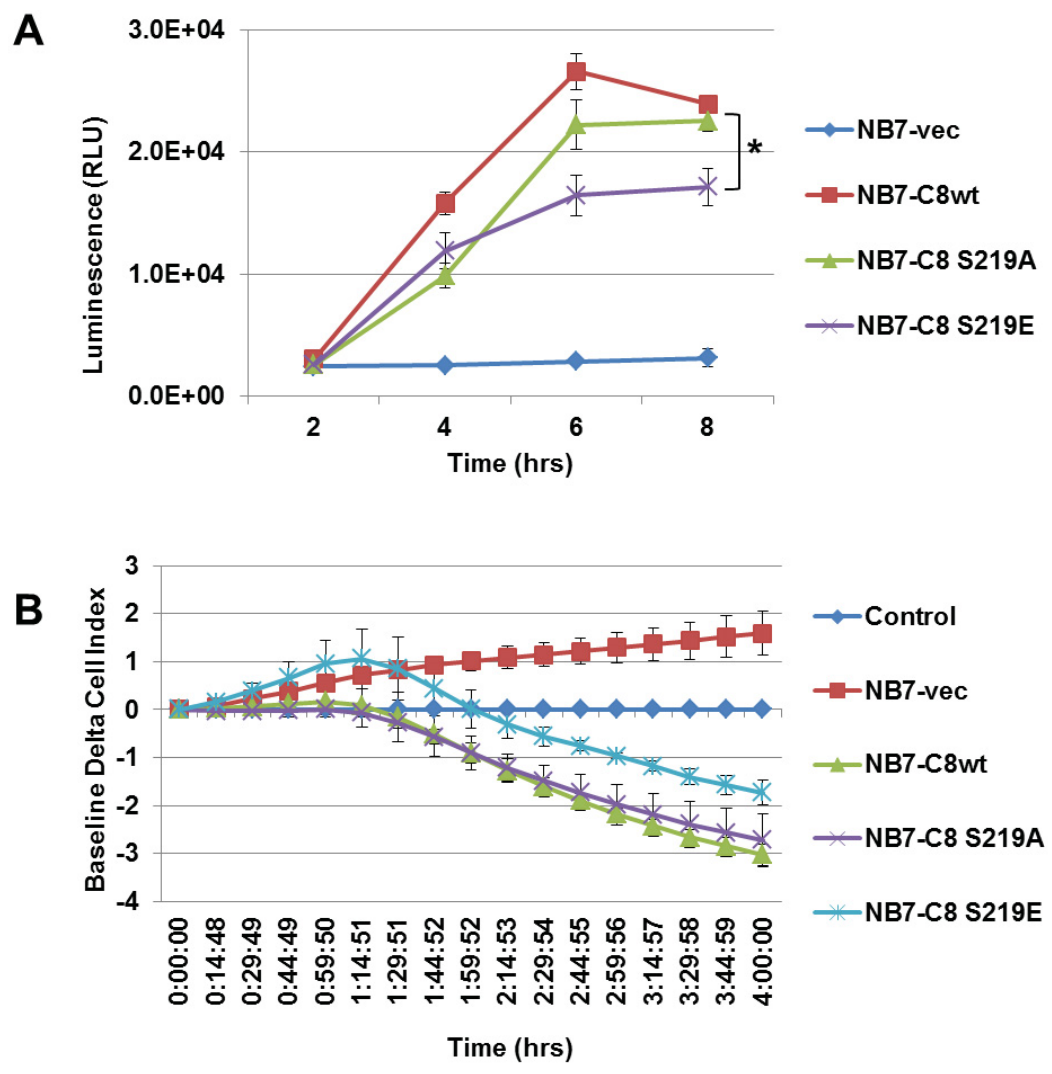

Figure 3-4. Phosphorylation at $\mathbf{S 2 1 9}$ suppresses caspase-8-mediated apoptosis.

NB7 cells, deficient or expressing casp8 (wt, A219, E219), were treated with TRAIL [50 $\mathrm{ng} / \mathrm{mL}]$ and MG132 $[1 \mu \mathrm{M}]$. (A) The enzymatic activity of casp8 was analyzed using a luminogenic cleavage substrate. Luminescent signal was measured at indicated times (*, $\mathrm{P}<0.004$ ). (B) The catalytic activity of casp8 was measured using the xCELLigence system which measures cellular impedance in real time. Cells are seeded at confluency and allowed to adhere to biosensor plated wells. Confluent wells have the highest level of cellular impedance. Cell death decreases the impedance. The difference of impedance over time was measured at indicated times. Control cells were NB7 cells expressing wt casp8 grown under normal conditions. Experiments were performed in triplicate. Error bars, \pm s.e.m. 
A
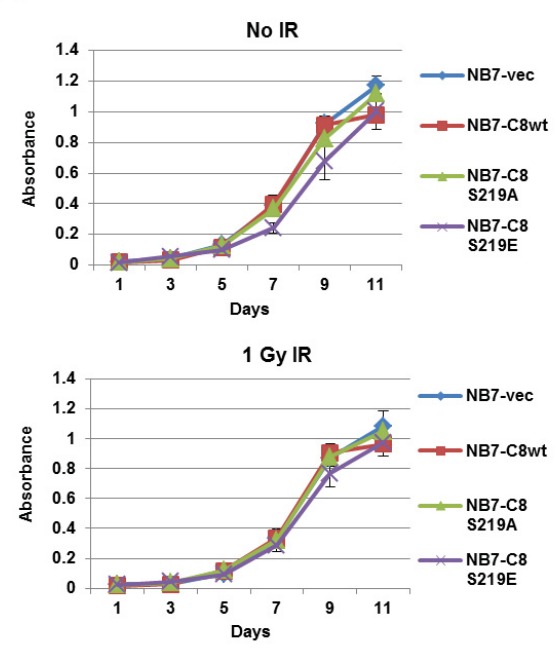

5 Gy IR
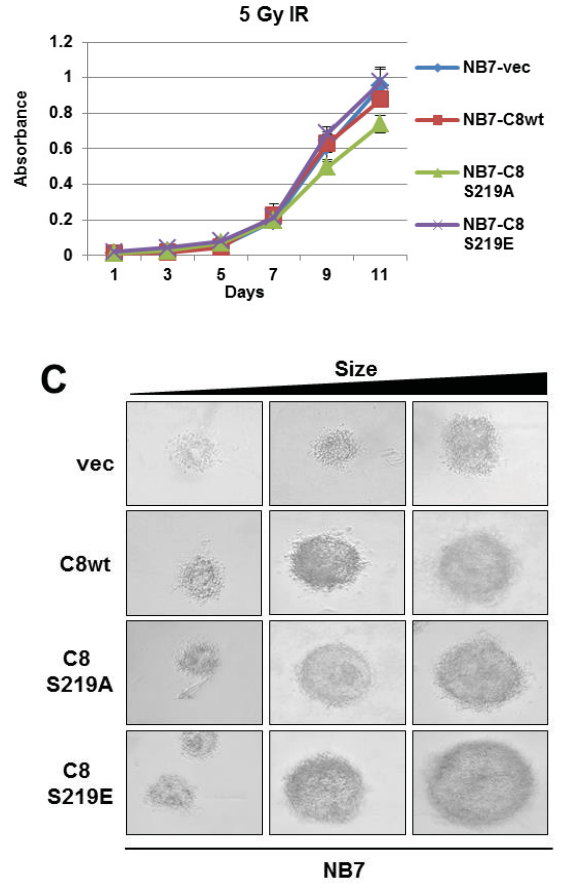

B
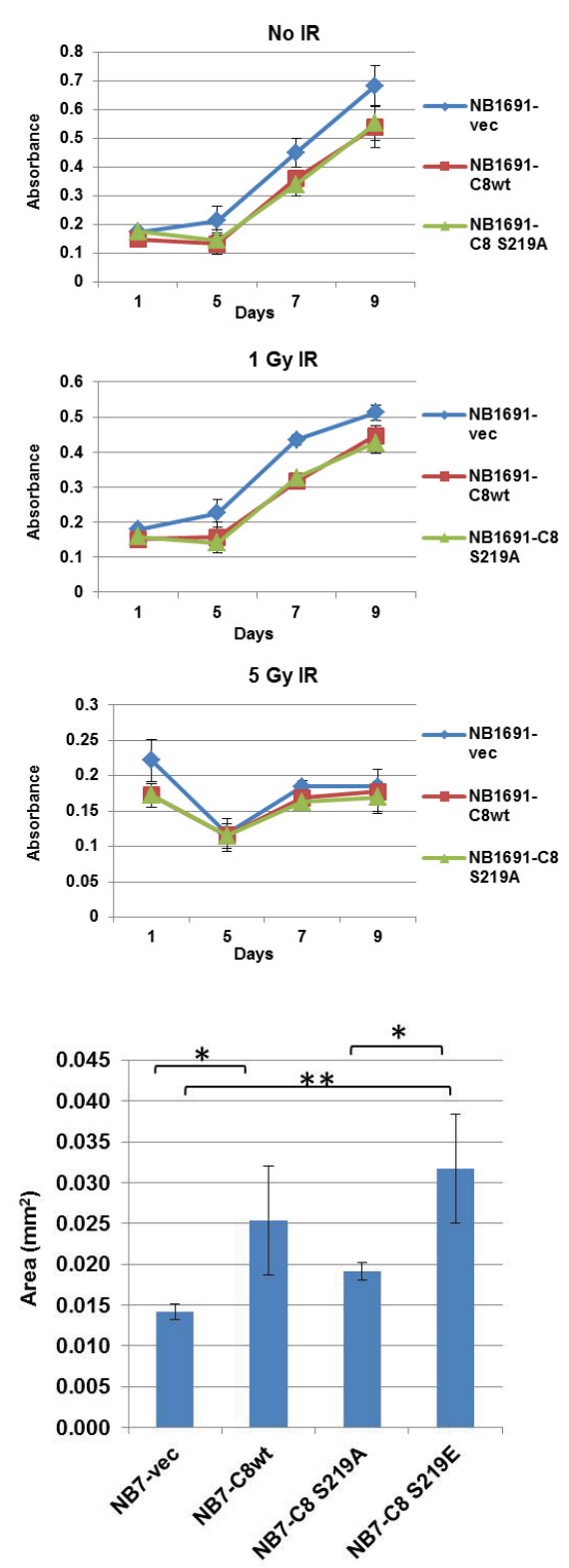

Figure 3-5. Phosphorylation increased colony formation and growth.

(A, B) Proliferation of both NB7 and NB1691 cells expressing the various casp8 constructs were exposed to various levels of IR and analyzed by MTT. (C) NB7 cells expressing casp8wt or a mutant form of casp8 and NB7 cells deficient for casp8 were suspended in a collagen matrix. Cells were maintained in matrix for 3 weeks and cultured under regular culture conditions. Colonies were counted and analyzed for size using the ImageJ Software. Experiment was performed in triplicate (*, $\mathrm{P} \leq 0.02$ and **, $\mathrm{P}<0.002)$. Error bars, \pm s.e.m. 
the role of casp8 in tumorigenesis may promote proliferation and be dependent on the microenvironment. In addition, NB7 casp8wt and S219E cells demonstrated an increased affinity for adhesion to collagen coated plates, suggesting a possible mechanism between casp8 and integrins (Figure A-2B). Stupack et al. demonstrated by coimmunoprecipitation that casp8 associates with integrins, while Barbero et al showed that casp8 is phosphorylated upon integrin ligation; both studies supporting casp8 involvement with integrins.

\section{Role of caspase-8 phosphorylation in NB tumorigenesis}

We previously demonstrated that casp8 enhances tumorigenesis of NB cells and have shown that casp 8 phosphorylation suppresses the enzymatic activity. Therefore, we hypothesized that phosphorylation at S219 may modulate casp8 function within the cell thereby altering the proapoptotic function of casp8. To investigate the possible role of casp8 phosphorylation in tumorigenesis, we orthotopically injected our NB7 cells expressing the nonphosphorylatable and phospho-mimic mutants of casp8 and our NB1691 cells expressing only the nonphosphorylatable casp8 mutant. In NB7 cells at the seven week time point, we noticed no significant difference between the two mutant versions of casp8 in comparison to casp8wt (Figure 3-6A). In addition, NB1691 cells expressing the alanine point mutation revealed no significant difference in tumor growth when compared to vector control and casp8wt tumors (Figure 3-6B). Analysis of overall survival of tumor bearing mice confirmed previous results demonstrating that phosphorylation does not appear to affect NB tumor growth or overall survival in this model (Figure 3-6C and D). Figure 3-6E is a representative image of the bioluminescent images obtained and quantified for the previously mentioned figures (Figure 3-6E). We also injected NB7 cells with both the S219A and S219E pointmutations into the flank of nude mice at the same cell concentration as our orthotopic model. Mouse flank tumors were analyzed weekly by bioluminescence for ten weeks. Tumor growth data from our flank injected tumors were similar to the para-adrenal xenografts (Figure A-2C).

Previously, we noticed a significant difference in the ability of casp8 to enhance tumor engraftment and growth; hence we examined our NB7 and NB1691 cells expressing the mutant construct of casp8 for variances in tumor engraftment. In support of our bioluminescent data, no significant differences were observed in overall tumor engraftment (Figure 3-7A and B). Figure 3-7C and D are representative images of five randomly selected tumors derived from the indicated cell lines demonstrating that overall tumor engraftment and growth was not enhanced or decreased due to phosphorylation

(Figure 3-7C and D). Xenografts derived from either NB7 casp8 S219A or S219E were analyzed by hematoxylin and eosin (H\&E) or probed for phospho-Histone H3. We quantified positive nuclei using the Aperio Spectrum ${ }^{\mathrm{TM}}$ software (Figure 3-7E). No significant differences were observed between the different mutant casp 8 constructs. 

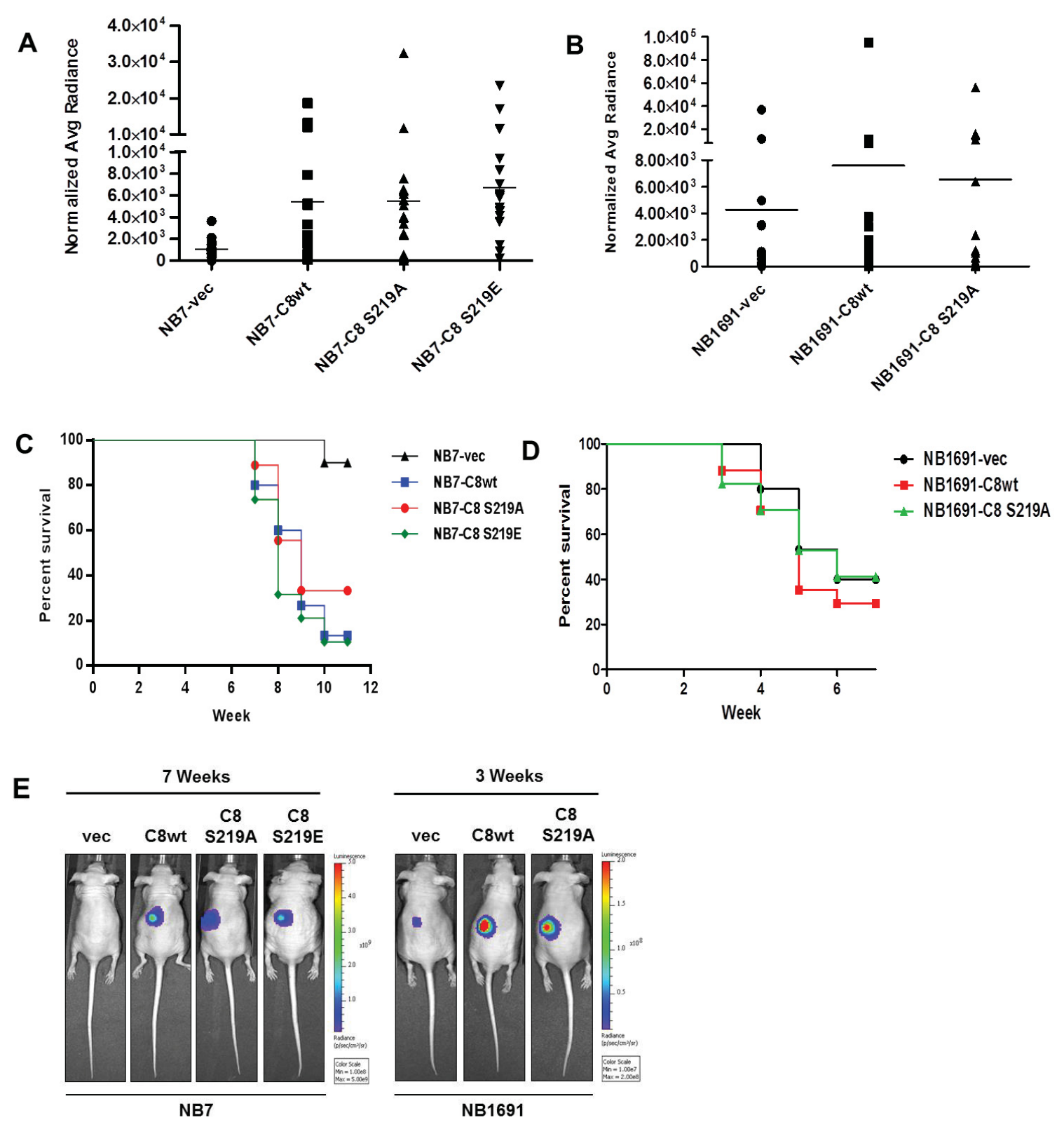

Figure 3-6. Phosphorylation of caspase-8 did not enhance tumorigenesis.

NB7 and NB1691 cells reconstituted with either nonphosphorylatable (S219A) or phospho-mimic (S219E) casp8 were orthotopically injected into the para-adrenal region of nude mice. Comparison of NB cells deficient for casp8, expressing wt, or mutant casp8. (A, B) Representation of the normalized bioluminescence imaging in orthotopically injected mice derived from NB7 or NB1691 cells expressing the indicated constructs of casp8. (C, D) Survival curve of tumor bearing mice. Once mice reached tumor burden they were removed from the study. (E) Representative bioluminescent images of tumor bearing mice. 


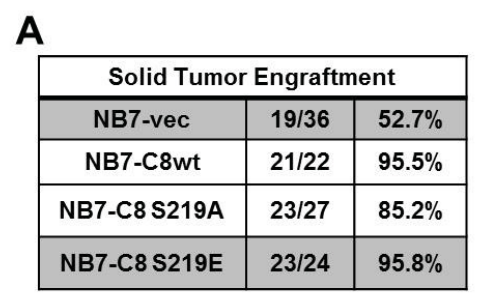

B

\begin{tabular}{|c|c|c|}
\hline \multicolumn{3}{|c|}{ Solid Tumor Engraftment } \\
\hline $\begin{array}{c}\text { NB1691- } \\
\text { Vec }\end{array}$ & $10 / 15$ & $66.6 \%$ \\
\hline $\begin{array}{c}\text { NB1691- } \\
\text { C8wt }\end{array}$ & $13 / 17$ & $76.4 \%$ \\
\hline $\begin{array}{c}\text { NB1691- } \\
\text { C8 S219A }\end{array}$ & $13 / 17$ & $76.4 \%$ \\
\hline
\end{tabular}

C

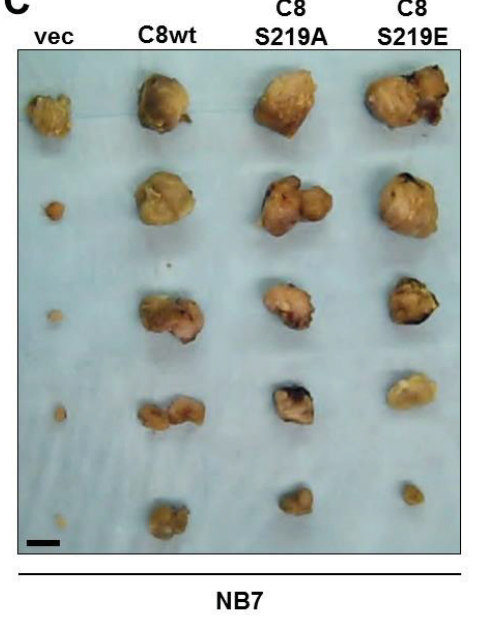

D

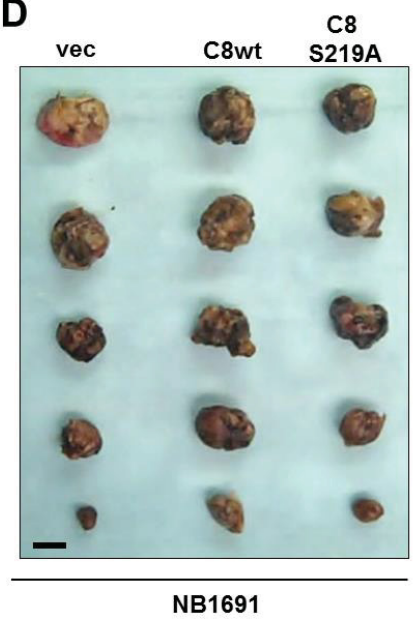

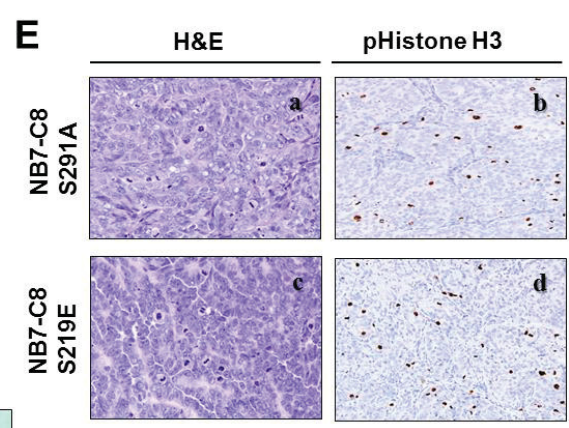

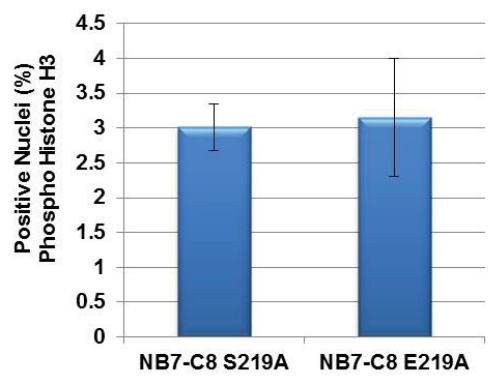

Figure 3-7. Phosphorylation did not affect tumor engraftment.

(A, B) After 10 weeks for NB7 and 6 weeks for NB1691 xenografts, mice not having reached tumor burden were euthanized and analyzed visually for solid tumor engraftment. Comparison to NB7 and NB1691 with and without casp8wt as previously shown. (C, D) Orthotopic xenograft tumors randomly selected from the indicated cell lines. Scale bar, $1 \mathrm{~cm}$. (E) Xenografts of NB7 cells reconstituted with either nonphosphorylatable mutant $(\mathbf{a}, \mathbf{b})$, or a phospho-mimic casp8 mutation $(\mathbf{c}, \mathbf{d})$ were transplanted into the para-adrenal region of nude mice and hematoxylin and eosin stained (a, c) or stained for phospho-Histone H3 (b, d). Quantification of positive phosphoHistone $\mathrm{H} 3$ staining from five representative tumors. 


\section{Regulation of caspase-8}

To determine in our orthotopic xenograft model if casp8 expression is affected by phosphorylation, we performed western blot analysis on tumors derived from NB7 casp8 S219A and S219E cells (Figure 3-8A and D). Quantification of casp8 expression levels was determined by densitometric analysis and normalized to the representative GFP expression and compared to the parental casp8 positive cell line

(Figure 3-8B and E). Densitometric analysis revealed that casp8 phosphorylation did not affect expression levels in our xenograft model. mRNA expression levels of casp8 were compared to the housekeeper gene, ubiquitin (Figure 3-8C and F). These data are similar to our casp8wt studies and demonstrate that under these conditions and in this model that there is no significant difference, biologically, by phosphorylation of casp8 at S219.

\section{Discussion}

In earlier studies, we demonstrated that the catalytic inactivation of casp 8 enhanced the tumorigenic property of casp8 positive NB7 cells. Therefore, we questioned as to how the enzymatic activity of casp8 could be modulated in vivo; thereby elucidating a possible biological switch of casp8, altering casp8 to a transducer of prosurvival signaling events. Literature searches revealed that casp8, as well as other caspases, have been shown to be phosphorylated which subsequently led to a decrease in the enzymatic potential of caspases, in general. Overall, we hypothesized that the function of casp 8 may be modulated by phosphorylation; thereby altering the balance between the canonical apoptotic and nonapoptotic functions of casp8. This hypothesis is supported by work demonstrating that by rendering casp 8 catalytically inactive via point mutation, that the embryonic lethality of casp 8 knockout could be rescued. During the process of wound healing, casp8 is preferentially silenced in sites proximal to the wound and later restored; thus demonstrating a mode of casp8 regulation and control. During our investigation of possible phosphorylation sites that could modulate the catalytic function of casp8, a large-scale proteomic study was published which suggested that ATM induces the phosphorylation of casp8 upon DNA DSB [106]. Therefore, we analyzed the role of this phosphorylation site in regard to the role of casp8 in apoptosis and tumorigenesis.

Detection of phosphorylated casp8 required immunoprecipitation of casp8 pulldown followed by a secondary pulldown of phosphorylated casp8 using an antibody raised against the ATM phosphorylation site of BID (S78). Prior to the post-translational analyses in NB, we determined whether the S219 residue of casp8 was evolutionarily conserved (Figure A-1A). Interestingly, S219 was conserved in casp8 in all species analyzed ranging from human to mouse. The SQ ATM recognition epitope was absent only in dog and mouse. From an evolutionary stand-point, this would argue against the possible phosphorylation event of casp8 by ATM plays an essential role in survival, demonstrating only to be evolutionarily conserved in higher mammals (primates). To validate that the $\mathrm{pBID}$ antibody detects phosphorylated casp8, we designed two peptides 

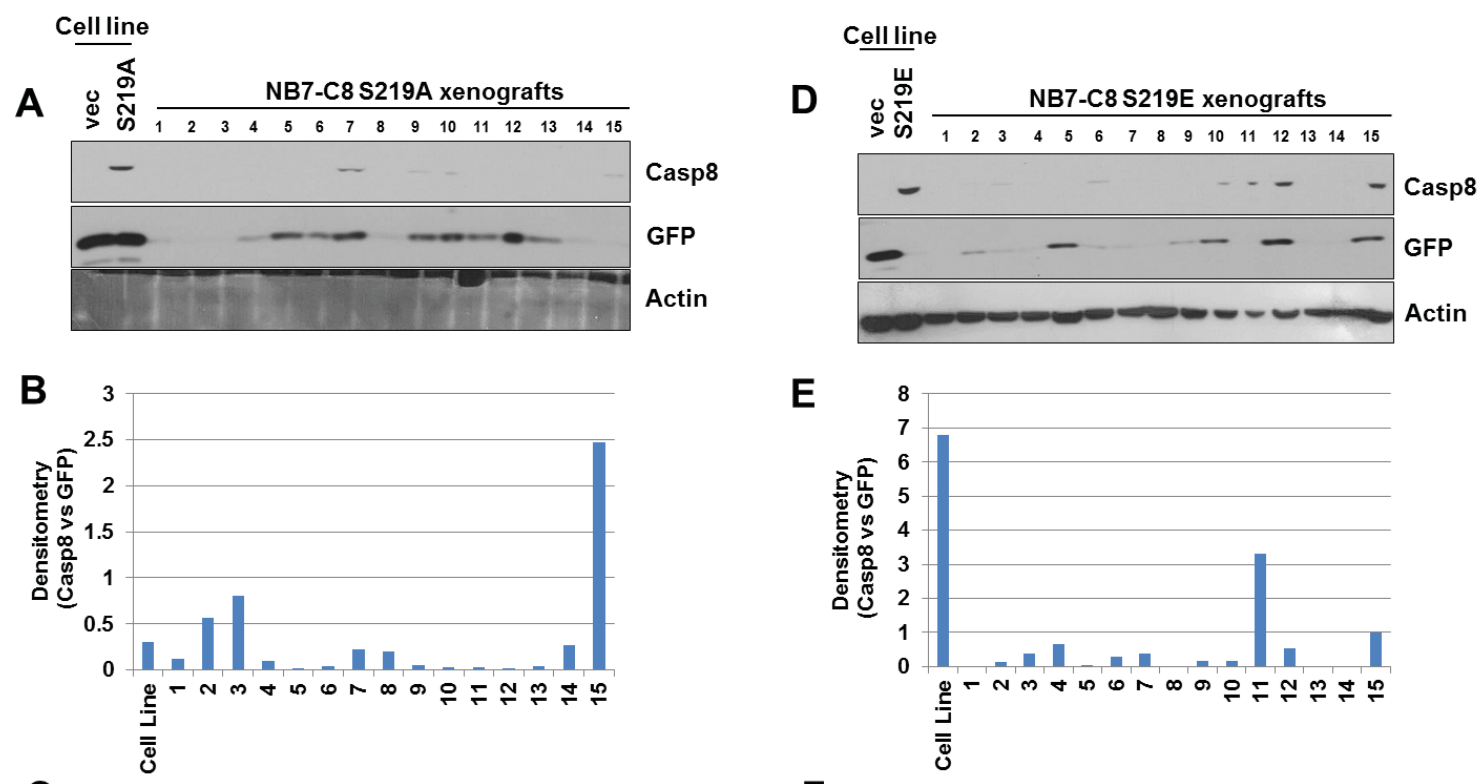

C

$\mathbf{F}$
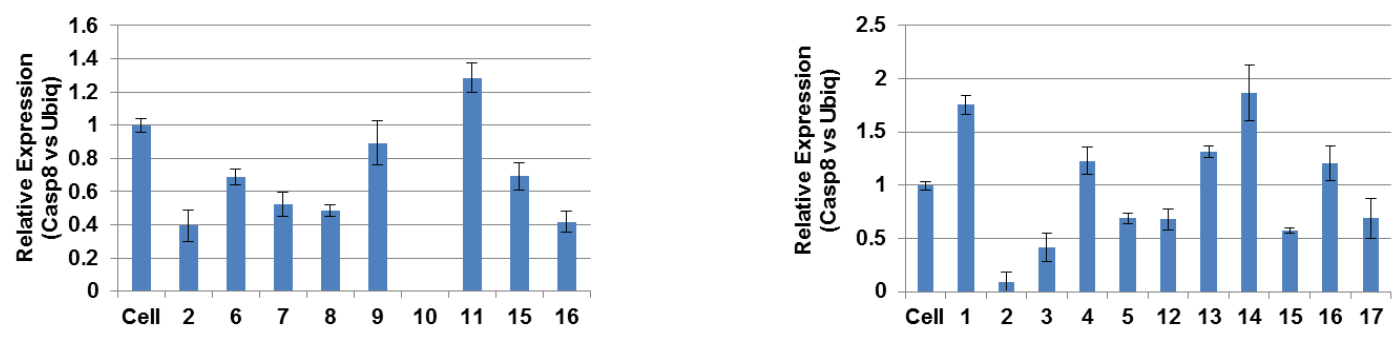

Figure 3-8. Posttranslational regulation of caspase-8 in S219A and S219E mutated caspase-8 expressing NB7 cells.

Caspase-8 expression in tumor xenografts derived from NB7 cells expressing casp8 S219A (A) and S219E (D). Control lanes consist of parental cell lines reconstituted with either vector only or indicated casp8 construct. Xenografts expressing the nonphosphorylatable (B) and the phospho-mimic (E) casp8 constructs were analyzed by densitometry for casp8 expression and normalized to the representative GFP expression level and compared to control cell line. Relative expression of casp8 mRNA normalized to ubiquitin in S219A (C) and S219E (F) xenografts. Xenograft numbers are the same for each experiment Error bars, \pm s.d 
comprising six to seven amino acids upstream and downstream of the human casp8 SQ site. To represent a positive control, one peptide was designed with a phospho-serine residue while the other peptide served as a negative, nonphosphorylated control. Peptides were run on an SDS-PAGE gel and analyzed for recognition by the phospho-BID antibody. Our analysis revealed that the antibody clearly recognized the casp8 ATM epitope when compared to our nonphosphorylated peptide control (Figure A-1B and C). Furthermore, when we compared BID and casp8 ATM epitopes (S78 and S219, respectively) we find that sequences downstream of SQ are highly conserved. Analysis of additional essential residues upstream and downstream required for ATM-mediated phosphorylation of serine revealed no other key residues; thus supporting that both the BID and casp8 ATM epitopes may be recognized by the same antibody.

We have confirmed casp8 phosphorylation using various cell lines with either endogenous or reconstituted wild-type or mutant casp8. Using a well-known and potent inhibitor of ATM and A-T cell lines deficient for ATM expression, we determined that ATM, not ATR, modulates casp8 upon DNA damage. In NB7 cells we confirmed that UV radiation did not promote casp8 phosphorylation. Surprisingly, casp8 was phosphorylated in NB1691 cells upon exposure to UV. We suspect that genetically the NB1691 cells may be more sensitive to DNA damaging agents; thereby leading to ATM activation and casp8 phosphorylation. This phenomenon of casp8 phosphorylation upon UV DNA damage would be interesting to investigate in other NB cell lines. Cell proliferation and cell cycle analysis post UV exposure may support our assumption that NB1691 are more sensitive to DNA damaging UV radiation. Active ATM was indicated by western blot in both NB7 and NB1691 cells exposed to both IR and UV suggesting still the possibility that ATM may be inducing the phosphorylation of casp8. In addition, we demonstrated in human NB cell lines that upon DNA damage that ATM translocates to the cytoplasm and mediates the phosphorylation of casp8 at S219. Prior research has demonstrated that a small subset of ATM may be cytoplasmic or may translocate to the cytoplasm [192-194]. These studies were performed in cell lines which may acquire nonphysiological functions due to culture conditions. To our knowledge, there are no published results demonstrating, in vivo, that ATM is cytoplasmic. We must take into account that in vivo modeling may also present with slight genetic differences in protein signaling and protein-protein interactions which may alter some of the protein functions and cell phenotypes. In our model, ATM-mediated phosphorylation of casp8 upon DNA damage would imply that ATM may regulate apoptosis by either promoting apoptosis due to extreme DNA damage or suppressing apoptosis in order to allow cells to repair damaged DNA. In the case of ATM-mediated phosphorylation of BID, the latter possibility is true. Therefore, to elucidate the biological significance of casp8 phosphorylation in regard to promoting death receptor mediated apoptosis, we exposed our cells to TRAIL and MG132 and quantified the cleavage of a proluminogenic casp8 substrate. These experiments determined that upon phosphorylation, the catalytic activity was suppressed. This is supported by previous results demonstrating that other kinases such as Src, Fyn, Lyn, RSK6 and p38 MAPK suppress casp8 catalytic activity [107]. Interestingly, we noticed that there was a moderate suppression of TRAIL-induced apoptosis, representing approximately a two-fold reduction when normalized to controls. Similar findings have been previously reported, indicating that phosphorylation does not 
abolish but merely slows that activity of casp8 allowing, in our model, for ATM to suppress apoptosis. Additional research investigating whether this phosphorylation event suppresses apoptosis under different inducers of extrinsic apoptosis, namely FAS and TNF- $\alpha$, may further confirm the functionality of casp8 phosphorylation. Examination of the $\mathrm{S} 219$ residue of casp8, reveals that the serine residue is proximal to a key aspartic acid residue at 216 (D216). Autocleavage of these aspartic acid residues stabilizes and promotes full activation of casp8 and phosphorylation may block cleavage at D216. Furthermore, phosphorylation may also induce conformational changes within the linker region; thereby blocking the activation site upon casp8 homodimerization.

Prior to orthotopically injecting our mutant casp8 expressing cell lines, we analyzed the cells in a 2D environment for cell growth. Interestingly, we noticed no significant difference in growth kinetics either by MTT, to measure metabolic rate, or cell cycle analysis using propidium iodide (PI) and flow cytometry (Figure A-3). In our xenograft models we noticed very dramatic differences in NB7 cells with and without casp8. To address the contribution of extracellular matrix in the regulation of casp8 and consequently cell proliferation and survival, we suspended NB cells in a collagen matrix and colony formation. After three weeks, NB7 cells expressing casp8, independent of wild-type or mutant, demonstrated significantly larger colonies. Our 3D environment recapitulated our xenograft data and showed that casp8 not only enhanced colony formation but cell growth within a 3D environment. Unfortunately, attempts to extract colonies from the collagen matrix were unsuccessful. Additional work characterizing individual colonies for casp8 expression levels will be necessary to further challenge the working model that casp8 is selected against in tumors. Work by Stupack et al. demonstrated that casp8 associates with integrins [145]. Taken together with our orthotopic and tail vein xenograft models and our 3D cell growth data, we propose that casp8 may function with integrins to transduce cell survival.

Xenograft studies revealed that the nonphosphorylatable and phospho-mimic casp8 mutants in NB7 and NB1691 did not significantly affect NB tumorigenesis. Caspase- 8 enhanced NB7 tumorigenesis independent of whether it was wild-type or mutant casp8, thus giving support for our previous work. We examined, by IHC, the xenografts derived from the NB7 cell line expressing the various casp8 constructs in order to determine if significant differences were observed. Unfortunately, no significant differences could be elucidated between the different casp8 constructs either by H\&E, casp3, phospho-Histone H3, and CD31. No apparent differences were noted in NB1691 cells reconstituted with either casp8wt and nonphosphorylatable casp8. In regard to the overall survival and engraftment rates, independent of the phosphorylation status of casp8, revealed to have no effect on overall survival. This observation may be due to the decreased expression of casp8, as discussed later, or the phosphorylation of casp8 at S219 may be an enhancer; thus phosphorylation of S219 promotes the phosphorylation at other sites of casp8 thereby initiating cell signaling. Such is the case where an unknown kinases phosphorylates casp8 at Y293, which promotes additional phosphorylation of Y397 and Y465 by Lyn kinase [107]. Therefore, ATM-mediated phosphorylation may just be a suppresser and regulator of apoptosis. Additional research is required to fully elucidate the potential biological mechanism of casp8 phosphorylation at S219. In 
addition, our tumor models may not be sufficiently sensitive to see minor differences conferred by phosphorylation of casp8. In this study we focused on casp8

phosphorylation as a potential mechanism to enhance NB tumorigenesis and developed studies to test tumor growth and development; however, the phosphorylational event of casp8 by ATM may have significance relevant to other key biological pathways not necessarily affecting tumorigenesis. Therefore, to fully understand casp8 and casp8 modulation in all facets of NB, additional research is required.

\section{Materials and Methods}

\section{Cell lines}

Human neuroblastoma, NB5, NB7, NB16 and NB1691 cell lines have been previously described $[17,128]$. In brief, neuroblastoma (NB5, NB7, NB16, NB1691) cell lines were established by St. Jude Children's Research Hospital. All NB cell lines including SK-N-AS were maintained in RPMI 1640 supplemented with 10\% FBS, 1\% glutamine and $0.1 \%$ gentamicin (Invitrogen, Grand Island, NY). 293T were maintained in DMEM supplemented with 10\% FBS, 1\% glutamine. All cells were maintained in a humidified atmosphere of $5 \% \mathrm{CO}^{2}$ at $37^{\circ} \mathrm{C}$. NB7 and NB1691 cell lines were reconstituted with casp8 using the pMSCV_IRES_GFP retroviral vector. Retrovirus supernatants were prepared as previously described [17, 172]. Briefly, NB cells were cultured with viral supernatants plus polybrene $[4 \mu \mathrm{g} / \mathrm{ml}]$. Viral supernatant was replaced with fresh supernatant every 2 hrs for 6 total hours. Infections were allowed to occur for a total of 72 hours at $37^{\circ} \mathrm{C}$. After 72 hours, cells were sorted for GFP expression. Collected cells were cultured for two weeks prior to being sorted again for GFP expression. Luciferase expression in NB7 cells was accomplished using the pMSCV_Luc_IYFP construct packaged in the previously described retroviral transduction method. For NB1691 cells, $3 \times 10^{6}$ cells were electroporated twice, with a 10 second interval between voltages, at 200 volts with $30 \mu \mathrm{g}$ of the pMSCV-LucSh-zeocin construct and selected for zeocin resistance $[100 \mu \mathrm{g} / \mathrm{mL}]$.

\section{Reagents}

Mouse anti-casp8 (C15) was a gift from Dr. M. Peter [158]. Goat anti-actin (C11), goat anti-lamin B (M-20), mouse anti-alpha tubulin (TU-02) were obtained from Santa Cruz Biotechnology, anti-rabbit GFP (A-11122) was from Invitrogen, anti-rabbit BID (2002), cleaved casp8 (D391) and rabbit phospho-ATR at Ser428 (cat\# 2853P) were from Cell Signaling. Rabbit anti-ATM (Ab-3) was purchased from Calbiochem, rabbit anti-phospho-BID (S78) (A300-528A) from Bethyl Laboratories, rabbit anti-phosphoATM at Ser1981 (EP1890Y, Epitomics, Burlingame, CA) ATM inhibitor, Ku-55933 was purchased from Tocris Bioscience (Minneapolis, MN). All antibodies were used as recommended by manufacturer. Caspase- 8 mutations were developed by the QuikChange site-directed mutagenesis kit (Stratagene, La Jolla, CA) and manufacturer's 
recommendations followed. Mouse trueblot was purchased from Rockland Scientific (18-8817-33).

\section{Immunoblotting}

Cells were lysed in Nonidet P-40 lysis buffer (20 mM Tris (pH 7.5), $150 \mathrm{mM}$ $\mathrm{NaCl}, 1 \mathrm{mM}$ EDTA, and $0.5 \%$ Nonidet P-40) plus $1 \mathrm{mM}$ PMSF, protease inhibitor cocktail (Roche, Mannheim, Germany) and phosphatase inhibitors ( $1 \mathrm{mM} \mathrm{NaF}, 1 \mathrm{mM}$ $\mathrm{Na}_{3} \mathrm{VO}_{4}$, and $1 \mathrm{mM}$ beta-glycerophosphate) for 5 minutes on ice followed by sonication. Lysates were centrifuged at 14,000 rpm for 10 minutes. Supernatants were removed and total protein was quantified using the Bradford assay (Bio-Rad, Hercules, CA). Proteins $(30 \mu \mathrm{g})$ were separated using SDS-PAGE analysis. Immunoblots were visualized using unlabeled primary antibodies and HRP-conjugated secondary antibodies. Protein expression was detected using enhanced chemiluminescence from GE Healthcare (Pittsburgh, PA). Quantification of band intensity was performed using the NIH ImageJ program.

Tumor samples were minced using a clean razor blade and suspended in ACK buffer $\left(0.15 \mathrm{M} \mathrm{NH}_{4} \mathrm{Cl}, 10 \mathrm{mM} \mathrm{KHCO}_{3}, 0.1 \mathrm{mM}\right.$ EDTA) for 1 minute on ice to lyse RBC and repeated in needed. Tumors were subsequently washed with PBS and suspended in RIPA buffer (150 mM NaCl, $50 \mathrm{mM}$ Tris ( $\mathrm{pH} 8.0)$, $0.5 \%$ sodium deoxycholate, $0.1 \%$ SDS, 1\% NP-40) plus protease inhibitor (Roche, Mannehim, Germany). Tumor samples were maintained in RIPA buffer for 5 minutes on ice and sonicated until tumor dissolved. Protein concentration was determined using the Bradford assay (Bio-Rad, Hercules, CA). Proteins $(30 \mu \mathrm{g})$ were separated using SDS-PAGE.

For immunoprecipitations, cells were lysed in Nonidet P-40 lysis solution 1 hour after exposure to either 10 Gy of irradiation using a ${ }^{137} \mathrm{Cs}$ source (Gammacell40extractor, MDS Norton) or $75 \mathrm{~J} / \mathrm{m}^{2}$ by a UV Strataliner 2400 (Stratagene). Caspase- 8 was pulled-down from $1 \mathrm{mg}$ of cell lysate using the C15 caspase- 8 antibody bound to protein $\mathrm{G}$ agarose beads (Pierce \# 20398) or cross-linking to $\mathrm{A} / \mathrm{G}$ agarose beads using a Pierce cross-linking kit (cat\#26147, Rockford, IL) and eluted from beads according to manufacture recommendation. Protein G agarose beads were washed in Nonidet P-40 lysis solution three times and suspended in SDS sampling buffer and boiled for 10 minutes.

\section{Colony formation}

NB7 cells were prepared in a single cell suspension at a concentration of $1 \times 10^{6}$ cells/mL. Collagen matrix was purchased from Millipore (cat \#ECM675, Billerica, MA). Cell slurry $(10 \mu \mathrm{L})$ was suspended in $100 \mu \mathrm{L}$ of collagen and seeded into a 96-well plate. Cells were maintained in cell culture media and incubated in a humidified atmosphere of $5 \% \mathrm{CO}_{2}$ at $37^{\circ} \mathrm{C}$ for 3 weeks. Colonies were randomly selected and imaged. Size of each colony was analyzed using the NIH ImageJ Software, colonies smaller than 0.01 
$\mathrm{mm}^{2}$ were excluded from study. Experiment was repeated in 3 times in triplicate with an average of 15 images obtained and analyzed per run.

\section{MTT}

Cells were cultured in 96-well plates under indicated conditions. At various time points, spent medium was removed and replaced with $20 \mu 1$ phenol red-free RPMI 1640 containing $5 \% \mathrm{FBS}$ and $0.5 \mathrm{mg} / \mathrm{mL}$ MTT for 3 hours at $37^{\circ} \mathrm{C}$. Isopropanol (200 $\left.\mu \mathrm{l}\right)$ was added to dissolve the MTT formazan crystals. Absorbance was measured at $570 \mathrm{~nm}$ and background at $690 \mathrm{~nm}$.

\section{In vitro kinase assay}

Purified recombinant caspase-8 protein was produced using the TNT Quick Coupled Transcription/Translation System (Promega, Madison, WI) following manufacturer recommendations. To investgate caspase- 8 phosphorylation in vitro, NB7 cells were lysed in Nonidet P-40 lysis buffer 1 hour after being irradiated at $10 \mathrm{~Gy}$. 500ug of total cell lysate were incubated with 2 ug of ATM antibody for 3 hours at $4{ }^{\circ} \mathrm{C}$. Protein $\mathrm{G}$ agarose beads $(15 \mu \mathrm{L})$ were then added to lysate and incubated overnight at 4 ${ }^{\circ} \mathrm{C}$. Beads were washed in lysis buffer and suspended in kinase buffer (40 mM Hepes (pH 7.4), 5 mM EGTA, 10 mM MgCl2, 100 mM NaCl, 2 mM Benzamide, $0.1 \mathrm{mM} \mathrm{NaF,}$ $0.1 \mathrm{mM} \mathrm{Na} \mathrm{VO}_{4}, 60 \mathrm{mM}$ beta-glycerophosphate, $1 \mathrm{mM}$ DTT). Combined $10 \mu \mathrm{L}$ of suspended beads with $5 \mu \mathrm{L}$ of purified recombinant caspase- 8 and $15 \mu 1$ of kinase master mix (4x kinase buffer, $1 \mathrm{mg} / \mathrm{mL}$ acetylated BSA, $1 \mathrm{mM} \mathrm{ATP}, 0.3 \mathrm{uL}{ }^{32} \mathrm{P}-\gamma \mathrm{ATP}$ ) and incubated at room temperature for 30 minutes. SDS loading buffer was added to each sample and boiled for 5 minutes and proteins separated on an SDS-PAGE gel and ${ }^{32} \mathrm{P}-$ $\gamma$ ATP-labeled proteins were detected by autoradiography.

\section{Cell fractionation}

Cells were washed in PBS, centrifuged and then suspended for 20 minutes on ice in lysis buffer (10 mM Tris ( $\mathrm{pH} 7.5), 5 \mathrm{mM} \mathrm{MgCl} 2,1 \mathrm{mM}$ DTT, $0.1 \%$ Nonidet P-40, 1 $\mathrm{mM}$ PMSF) plus protease inhibitor cocktail (Roche, Mannehim, Germany). For phosphorylation studies, phosphatase inhibitors were added (1 $\mathrm{mM}$ betaglycerophosphate, $1 \mathrm{mM} \mathrm{Na} \mathrm{VO}_{4}, 1 \mathrm{mM} \mathrm{NaF}$ ). Cells were then centrifuged for 10 minutes at $2000 \mathrm{rpm}$ and supernatant (cytosolic fraction) was saved. Pelleted fraction was washed again in lysis buffer and centrifuged. Supernatant was added to the cytosolic fraction and pellet was suspended in a secondary lysis solution of $10 \mathrm{mM}$ Tris ( $\mathrm{pH} 7.5), 5$

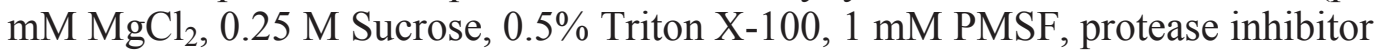
cocktail and $1 \mathrm{mM}$ beta-glycerophosphate, $1 \mathrm{mM} \mathrm{Na}_{3} \mathrm{VO}_{4}$, and $1 \mathrm{mM} \mathrm{NaF}$ for phosphorylation studies. Lysates were centrifuged for 5 minutes at $2000 \mathrm{rpm}$. Supernatants were discarded and pellets were suspended in a third lysate solution (10 $\mathrm{mM}$ Tris (pH 7.5), 0.25 M sucrose, $1 \mathrm{mM}$ PMSF, protease inhibitor cocktail, and $1 \mathrm{mM}$ 
beta-glycerophosphate, $1 \mathrm{mM} \mathrm{Na}_{3} \mathrm{VO}_{4}$, and $1 \mathrm{mM} \mathrm{NaF}$ ). Cell fraction pellets were sonicated and layered on top of a $0.88 \mathrm{M}$ Sucrose solution. Cell fractions were centrifuged through the sucrose gradient at $2000 \mathrm{rpm}$ for 20 minutes. The top layer was carefully removed and maintained as the nuclear fraction.

\section{RT-PCR}

Total RNA was extracted from xenograft tumors with an RNA kit (Qiagen, Valencia, CA) according to manufacturers' recommendations. Complementary DNA was prepared with the iScript cDNA synthesis kit (Bio-Rad, Hercules, CA) from $1 \mu \mathrm{g}$ of RNA. IQ Syber green Supermix (Bio-Rad) and oligonucleotide primers Casp8-F (5'AAGCAAACCTCGGGGATACT), Casp8-R (5'-GGGGCTTGATCTCAAAATGA), GFP-F (5'-CACATGAAGCAGCACGACTTCT), GFP-R (5'-AACTCCAGCAGGACCATGTGAT), Ubiq-F (5'-ACCTGACCAGCAGCGTCTGATATT), and Ubiq-R (5'TCGCAGTTGTATTTCTGGGCAAGC) were used to perform a two-step real-time PCR assay. Samples were denatured at $95^{\circ} \mathrm{C}$ followed by an annealing and extension step at $62^{\circ} \mathrm{C}$. Relative expression was quantified by the $\Delta \mathrm{C}_{\mathrm{T}}$ method and normalized to either GFP or human ubiquitin.

\section{Caspase-8 enzymatic assay}

The catalytic activity of caspase- 8 was assessed using a Caspase-Glo 8 Assay kit (Promega, cat\#G8200, Madison, WI) according to the manufacturer's instructions. Briefly, neuroblastoma cells were seeded in 96 -well plates at a concentration of $2.5 \times 10^{4}$ cells/well and allowed to adhere overnight. Cells were treated with $50 \mathrm{ng} / \mathrm{mL}$ of TRAIL and $1 \mathrm{uM}$ of MG132 [204]. Caspase-Glo 8 reagent was then added following manufacturer's recommendations and allowed to incubate for $1 \mathrm{hr}$ at room temperature. Luminescence of each sample was measured at $0,2,4,6$, and $8 \mathrm{hrs}$ using a plate reader. The growth kinetics of TRAIL $(50 \mathrm{ng} / \mathrm{mL})$ and MG132 $(1 \mu \mathrm{M})$ treated NB7 cells were examined using the Real Time Cellular Analyzer (RTCA) (xCELLigence system; Roche, Basel, Switzerland) which measures a cell's interaction or impedance of biosensors. Cells were seeded at an empirically determined concentration in a 16-well goldmicroelectrode E-plate and allowed to adhere overnight. Following adhesion, cells were treated with TRAIL (Prospec, East Brunswick, NJ) and MG132 (Millipore, Billerica, MA) and cell impedance was monitored every 5 minutes for 4 hours.

\section{Orthotopic xenograft}

Ultrasound-guided orthotopic xenograft procedures were previously detailed [154]. Briefly, cells were suspended in Matrigel (BD worldwide, Cat\#354234, San Jose, CA) at a concentration of $2 \times 10^{4}$ cells $/ \mu \mathrm{L}$ and kept on ice. Using a VisualSonics VEVO770 High Frequency Ultrasound system (VisualSonics, Toronto, Canada), a 22 gauge catheter was inserted into the para-adrenal space through the back muscle of anesthetized 
CD1 nude mice. A Hamilton syringe loaded with $10 \mu \mathrm{L}$ of cell suspension was guided steriotactically into the region between the kidney and adrenal gland and injected. For tumor growth analysis, mice were injected (intraperitoneal) with $3 \mathrm{mg} /$ mouse of Firefly D-Luciferin (Caliper Life Sciences) and imaged using the IVIS ${ }^{\circledR} 200$ imaging system. Bioluminescent images were analyzed using the Living Image 4.2 software (Caliper Life Sciences). Identical regions of interest (ROI) were used to determine the average radiance (photons $/ \mathrm{sec} / \mathrm{cm}^{2} / \mathrm{sr}$ ) of all images for all time points. For tail vein injections, cells were maintained in exponential growth and were suspended in serum free culture media at a concentration of $5 \times 10^{7}$ cells/mL. CB17/SCID mice were injected twice with $100 \mu 1$ of cell slurry with a 1 hour recovery time between injections. Mice were euthanized 9-10 weeks post injections and analyzed by full necropsy. Organs were analyzed for tumor cells using hematoxylin and eosin (H\&E), and for the neuronal markers synaptophysin, chromogranin A and PGP9.5. Digital calipers were used to measure tumor size. 


\section{CHAPTER 4. OVERALL SUMMARY AND FUTURE EXPERIMENTS}

\section{Summary}

Neuroblastoma survival rates have improved; however, despite advances in chemotherapies and treatments, patients diagnosed with advanced-stage NB have shown little to no improvement with less that $40 \%$ long-term survival rate [8]. Caspase- 8 has been shown to be silenced in up to $70 \%$ of advanced-stage NB patient tumors, while 30 $50 \%$ maintained casp 8 expression [17]. Significance of casp 8 deficiency decreased chemotherapeutic drug sensitivity and enhanced metastatic cell survival by preventing integrin-mediated cell death $[127,128,145,153]$. In contrast, casp8 expression enhanced cell migration, adhesion, and promoted ruffle and lamellipodial formation [88, 91, 149]. Caspase- 8 may also regulate cell cycle via the S6K pathway, NF-кB activation, and Erk1/2 activation $[86,87,96,101]$. These processes were determined to be independent of the enzymatic activity required for apoptosis. In chapter two we discussed and demonstrated for the first time additional tumorigenic properties of casp8. We determined using reconstituted casp8 in NB7 cells that casp8 enhances tumor engraftment and tumor growth. Extensive studies in NB1691 were not performed due to our preliminary studies demonstrating that casp8 did not enhance NB1691 tumor formation and/or growth. Additional studies investigating the genetic differences and titering our cell number used in our xenograft model may demonstrate possible differences in NB1691 with and without casp8 in NB tumorigenesis. Furthermore, we showed by rendering casp 8 catalytically inactive via point-mutation, that the enzymatic activity is not required for casp8-mediated tumor growth and formation. Surprisingly, the $\mathrm{N}$-terminal domain or prodomain of casp8 is essential for tumorigenesis. This would suggest that casp8 must bind an adaptor protein (FADD or TRADD) to mediate its prosurvival function and promote tumorigenesis. Supporting other published data, casp8 was silenced at an unknown time point during tumorigenesis. This would suggest a new avenue for additional research, to define the temporal regulation of casp 8 expression using our orthotopic xenograft model. In human patient samples, casp8 is predominately silenced by hypermethylation; yet, in our xenograft models NB cells seemingly regulate casp8 at either the translational or posttranslational level. Additional experiments including pulse chase studies, polysomal preps, and ubiquitin bloting may elucidate the exact level of casp8 regulation in our NB xenograft models. Since casp8 expression in NB7 cells enhances tumorigenesis and casp8 expression is downregulated by ten weeks, casp 8 may be essential during the early phases of tumorigenesis and not be required once tumor establishment has occurred. Analysis of xenografts harvested at different time points post-transplantation should provide additional insight into the mechanism by which casp8 contributes to tumorigenesis.

Previous research showed that protein kinases including Src, ATM, p38 MAPK and Cdk1, modulate and regulate casp8 activity and cellular function. In general, phosphorylation reduces the enzymatic activity of casp8. In hepatocellular carcinoma, breast and colon cancer elevated levels of phosphorylated casp 8 were found, suggesting that the phosphorylation of casp8 may enhance tumorigenesis and/or metastasis [101, 
103]. Our research suggests that casp8 is phosphorylated by ATM at S219 upon DNA damage. This was demonstrated by extensive western blot analysis using various DNA damaging reagents, an ATM inhibitor, and human fibroblasts (null for ATM). Characterization of phosphorylated casp8 in NB tumorigenesis revealed no significant differences in either tumor growth or establishment. Independent of the phosphorylation status of casp8, NB7 cells expressing casp8 had a higher frequency of tumor engraftment and tumor growth, supporting our previous data. Phosphorylation of casp8 at S219 did show a suppressive effect of its enzymatic activity. This may be a potential model for ATM to regulate extrinsic apoptosis upon DNA damage; which would allow cells to repair DNA. Furthermore, independent of the phosphorylation status of casp8, western blot analysis revealed that casp8 is downregulated in late stage NB.

Based on this work and work performed by others, we suggest that the overall function of casp8 in NB may be at the early stages of tumorigenesis by enhancing tumor cell establishment and proliferation. We have demonstrated that casp8 expression in NB7 cells enhanced tumor engraftment in our orthotopic and tail vein mouse models. Studies suggest that cells positive for casp8 along the periphery of the tumor are more sensitive to apoptosis due to their involvement in IMD. Furthermore, seeding in a foreign secondary environment promotes casp 8 expression, suggesting that those cells that can effectively suppress casp 8 may be more metastatic. Consistent with this hypothesis casp8 expression in the secondary tumor, once established, is downregulated (Figure 4-1).

Interestingly, casp8 effects on tumor cell proliferation were only observed in a 3D environment, xenografts or a collagen matrix. Previous data demonstrated direct involvement of casp8 with integrins and casp8-mediated signaling induced by EGF stimulated integrins. Ligand binding to a death receptor promotes casp8 recruitment and subsequent activation leading to cell death. Stimulation of integrins based on cell environment may block casp8-mediated apoptotic signaling or promote casp8 noncanonical survival functions. Cells and tumor cells alike are constantly exposed to DNA damaging agents and stressors such as hypoxia, starvation, and ROS, which may lead to ATM activation and subsequent regulation of casp8. Though ATM-mediated regulation of casp8 only suppresses the extrinsic apoptotic pathway, this suppressive affect may enhance cell survival under stressful conditions (Figure 4-2). These findings suggest one potential mechanism as to why some tumors are less sensitive to DNA damaging chemotherapies.

Overall, casp8 appears to be a multi-functional protein playing key roles in two opposing processes; cell death and survival. The factors and signals that influence these processes likely involve changes in protein complexes, homodimerization versus heterodimerization, and modification of casp8. Elucidating the mechanism by which casp8 is regulated and functions in cancer will be important in developing new chemotherapeutics and improving outcome for patients with NB. 

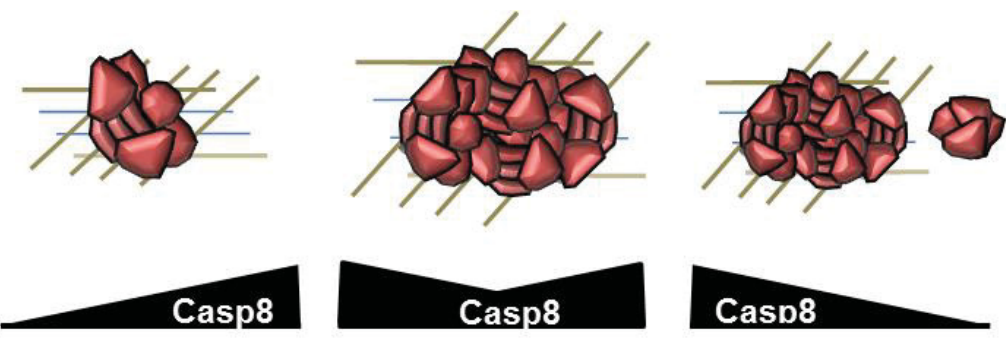

Casp8

PRIMARY

INVASION

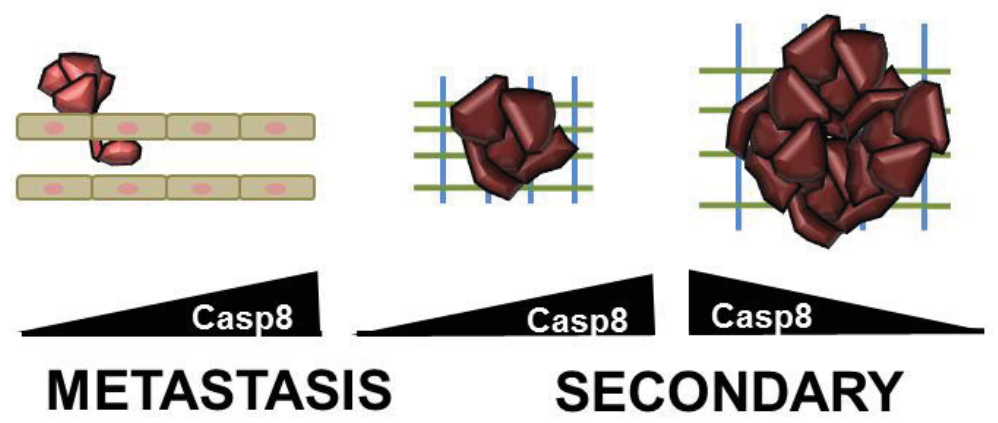

Figure 4-1. Role of caspase-8 in neuroblastoma.

A cartoon representation of the possible casp8 expression levels at various stages tumor development, as suggested in the literature and current research. 


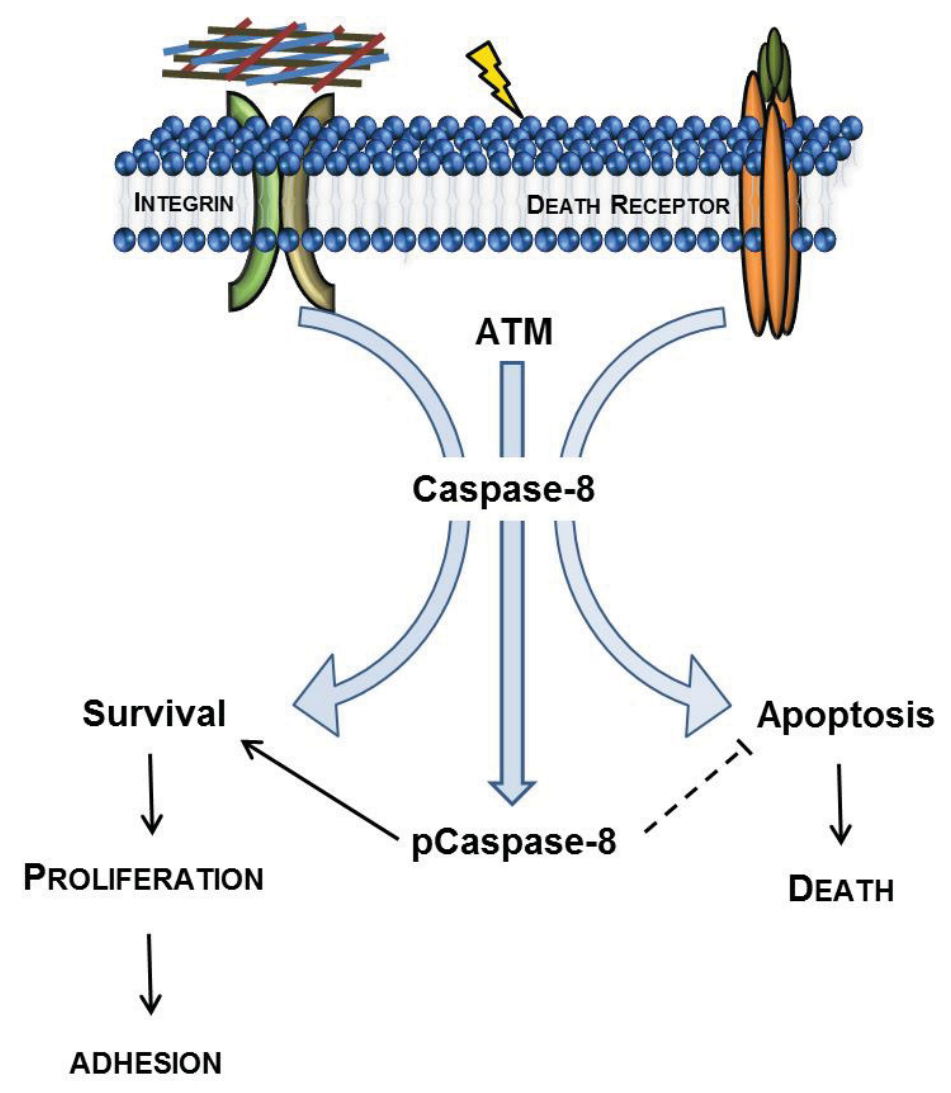

Figure 4-2. Model of caspase-8 induced signaling.

A model representing two potential modes of casp8 activation and their downstream signal transduction. 


\section{Future Directions}

\section{Mechanism of caspase-8 during tumorigenesis}

As previously stated, our research suggests that casp8 promotes early phase tumorigenesis by enhancing tumor establishment/colonization and growth of NB. To confirm the role of casp 8 in early primary tumor formation, we propose time course studies involving the extraction of primary tumor masses at specific stages post orthotopic injection into nude mice. These tumors would be monitored by ultrasound for tumor volume and by bioluminescence for growth kinetics. Simple western blot and quantitative real time PCR analyses of harvested tumors based on time points and size may elucidate when casp8 expression is downregulated. Elucidation of exact stages during tumorigenesis at which casp8 is downregulated may identify other key events (i.e. angiogenesis, growth, metastasis, etc) occurring during that time point which may give insight as to the possible mechanistic roles of casp8 in tumor growth.

Knockout of casp8 in mice is embryonically lethal due to malformation of the heart and poor vascularization, giving rise to the possibility that casp 8 may promote angiogenesis. Visually, we noted that casp 8 positive tumors were more angiogenic based on increased tumor vasculature in comparison to vector control NB7 cells. Unfortunately, we were unable to confirm these findings by stain for an endothelial cell marker (CD31). Expanding our endothelial and angiogenic probes to include CD34, CD105, vWf, and VEGF may validate our visual analysis of our NB xenografts. Including both the chick chorioallantoic membrane (CAM) and the Matrigel plug assay using NB cells with and without casp8 would further validate the potential role of casp8 in NB angiogenesis.

Our xenograft model suggests that casp 8 expression is downregulated by the end of our ten week study. This issue may be addressed by establishing tumor explants or cell line cultures short term in vitro and casp8 expression monitored by pulse-chase analysis with and without proteasomal inhibitors. Earlier attempts were made to determine if casp8 was downregulated by protein degradation as determined by western blot analysis for ubiquitin. This method was technically difficult due to the extremely high background levels of casp8 pull downs and poor ubiquitin antibodies.

Immunoprecipitation of tagged casp 8 may decrease background and allow for ubiquitin blotting.

The next critical aspect of characterizing the function of casp8 in tumorigenesis is to elucidate the mechanism and signaling pathways induced by casp8. Mechanistically, casp 8 has been shown to have a higher affinity for FLIP heterodimerization as opposed to homodimerization with casp8. It has been suggested that casp8 homodimerization transduces apoptosis while heterodimerization mediates cell survival, due to FLIP inability to induce the intersubunit cleavage events of casp8. Currently, we have constructed an $\mathrm{N}$ and $\mathrm{C}$-terminal FLAG tag construct of casp8 for pulldown experiments. This construct can be transiently transfected into our NB cell lines and analyzed under 
various stressors (i.e. hypoxia, IR, and lack of nutrients, etc) for heterodimerization. To investigate casp8 heterodimerization using FLAG to pulldown casp8 within a 3D environment, we would need to design tagged casp8 into a retroviral vector. Our studies have shown that cell culture within a 3D collagen matrix mimicked tumor xenograft studies. Therefore to investigate the dimerization status of casp8 within a 3D environment we would use a proximity ligation assay (PLA) [205]. This assay works similarly to FRET where only upon close proximity of the two proteins of interest promotes the fluorophore to become excited. Pulldown studies and PLA assay would identify the possible interactions of the known proteins to interact with casp8. In addition, if casp8 heterodimerization was shown to be relevant during colony formation, crossing FLIP knockout mice with casp8 knockout would demonstrate if the embryonic lethality of both casp8 and FLIP knockouts were dependent on their heterodimerization. David Wallach's lab demonstrated that by rendering casp8 catalytically inactive the embryonic lethality of casp $8^{-/-}$mice could be rescued. Essentially, heterodimerization of casp8 to FLIP suppresses the enzymatic activity of casp8 and essentially promotes a nonapoptotic response. Therefore, we would hypothesis that casp8/FLIP heterodimerization is essential in promoting the prosurvival and subsequent protumorigenic function of casp8.

As previously stated several strategies have been developed to upregulate and promote casp8 as a potential therapeutic target for cancer therapy. Since casp8 is most commonly epigenetically silenced in cancers, as opposed to mutated or deleted, treatment options include the use of 5-Aza, IFN- $\gamma$, and RA [116, 127, 139] Though inducing casp8 expression may present an attractive therapeutic target due to its apoptotic function, it may also promote survival. Research shows that casp8 expression can be elevated in some tumors and some malignant tumors may tolerate high levels of casp8. Furthermore, given the intense selective pressures present in the tumor microenvironment, upregulation of casp8 may promote the noncanonical prosurvival roles of casp8. In such situations, increasing casp 8 may be detrimental $[141,142]$. Additional studies driven to understand the potential role of casp 8 in chemotherapeutic responses would prove valuable in our xenograft model. In our current study, we studied casp8 in response to IR and demonstrated that casp8 was phosphorylated upon DNA damage; however, we were unsuccessful to test this phosphorylation event in our xenograft model. Previously, we irradiated mice bearing NB7 casp8wt xenografts and probed tumor lysates for phosphorylated casp8. Due to the high background it was difficult to determine if casp8 in our xenografts was phosphorylated. We propose that by tagging casp 8 with either FLAG or HIS may allow for cleaner IPs and easier detection of casp8. Detection of casp8 phosphorylation in vivo would enhance the clinical significance of casp8 phosphorylation upon DNA damage. In addition to IR, examination of other DNA damaging agents that are commonly used in the clinic (etoposide, cisplatin, etc.) may also clarify the potential role of casp8 in therapeutic resistance/sensitivity. 


\section{Phosphorylation of caspase-8}

My research has focused on primarily on S219 phosphorylation and its effect on cell survival and tumor growth. Caspase- 8 is posttranslationally modified at multiple sites. Prior to our investigation of ATM-mediated phosphorylation of casp8, we determined possible phosphorylation sites of casp8 using several online phosphorylation site calculators. Several of these sites, some of which had been previously shown in the literature, were mutated at the phosphorylation site. All constructs have been generated and confirmed by DNA sequencing. Previously, we planned to stably express these constructs in NB cells lines and analyzing the cells for cell growth in order to determine which sites produced the greatest phenotype in our orthotopic and tail vein tumor models. One additional measure we would add to these studies, based on previous experience, would be to grow these cells in a 3D matrix. Those sites which demonstrated either the greatest or lowest growth, as compared to vector only controls, would be analyzed for possible kinase involvement, mechanism of phosphorylation, and overall phenotype due to the phosphorylation status. Binding kinetics of phosphorylated casp8 to casp8, phosphorylated casp8, and FLIP would be analyzed based on the model by Oberst et al. using an inducible binding prodomain [56]. Phosphorylated casp8 at Y360 and at S387 have previously been determined in patient samples presenting with breast and colon cancer, suggesting that the phosphorylation of casp8 may enhance tumorigenesis and/or metastasis [101, 103]. Evaluation of casp8 phosphorylation at S219 in human patient samples would increase the validity and importance of casp8 in tumorigenesis. 


\section{LIST OF REFERENCES}

1. Gurney, J.G., et al., Infant cancer in the U.S.: histology-specific incidence and trends, 1973 to 1992. Journal of pediatric hematology/oncology : official journal of the American Society of Pediatric Hematology/Oncology, 1997. 19(5): p. 42832.

2. Rothenberg, A.B., et al., Neuroblastoma-remembering the three physicians who described it a century ago: James Homer Wright, William Pepper, and Robert Hutchison. Pediatric radiology, 2009. 39(2): p. 155-60.

3. Brodeur, G.M., Neuroblastoma: biological insights into a clinical enigma. Nature reviews. Cancer, 2003. 3(3): p. 203-16.

4. Schor, N.F., Neuroblastoma as a neurobiological disease. Journal of neurooncology, 1999. 41(2): p. 159-66.

5. Anderson, D.J. and R. Axel, A bipotential neuroendocrine precursor whose choice of cell fate is determined by NGF and glucocorticoids. Cell, 1986. 47(6): p. 1079-90.

6. Anderson, D.J., et al., Antibody markers identify a common progenitor to sympathetic neurons and chromaffin cells in vivo and reveal the timing of commitment to neuronal differentiation in the sympathoadrenal lineage. $\mathrm{J}$ Neurosci, 1991. 11(11): p. 3507-19.

7. Janoueix-Lerosey, I., G. Schleiermacher, and O. Delattre, Molecular pathogenesis of peripheral neuroblastic tumors. Oncogene, 2010. 29(11): p. 1566-79.

8. Maris, J.M., et al., Neuroblastoma. Lancet, 2007. 369(9579): p. 2106-20.

9. Matthay, K.K., et al., Phase II study on the effect of disease sites, age, and prior therapy on response to iodine-131-metaiodobenzylguanidine therapy in refractory neuroblastoma. Journal of clinical oncology : official journal of the American Society of Clinical Oncology, 2007. 25(9): p. 1054-60.

10. Shimada, H., et al., The International Neuroblastoma Pathology Classification (the Shimada system). Cancer, 1999. 86(2): p. 364-72.

11. Jiang, M.R., J. Stanke, and J.M. Lahti, The Connections between Neural Crest Development and Neuroblastoma. Cancer and Development, 2011. 94: p. 77-127.

12. Maris, J.M., et al., Evidence for a hereditary neuroblastoma predisposition locus at chromosome 16p12-13. Cancer Res, 2002. 62(22): p. 6651-8.

13. Maris, J.M. and K.K. Matthay, Molecular biology of neuroblastoma. Journal of clinical oncology : official journal of the American Society of Clinical Oncology, 1999. 17(7): p. 2264-79.

14. Look, A.T., et al., Cellular DNA content as a predictor of response to chemotherapy in infants with unresectable neuroblastoma. The New England journal of medicine, 1984. 311(4): p. 231-5.

15. White, P.S., et al., Detailed molecular analysis of $1 p 36$ in neuroblastoma. Medical and pediatric oncology, 2001. 36(1): p. 37-41.

16. Kogner, P., et al., Coexpression of messenger RNA for TRK protooncogene and low affinity nerve growth factor receptor in neuroblastoma with favorable prognosis. Cancer research, 1993. 53(9): p. 2044-50. 
17. Teitz, T., et al., Caspase 8 is deleted or silenced preferentially in childhood neuroblastomas with amplification of MYCN. Nat Med, 2000. 6(5): p. 529-35.

18. Brodeur, G.M., et al., Biology and genetics of human neuroblastomas. Journal of pediatric hematology/oncology : official journal of the American Society of Pediatric Hematology/Oncology, 1997. 19(2): p. 93-101.

19. Fulda, S., et al., MycN sensitizes neuroblastoma cells for drug-induced apoptosis. Oncogene, 1999. 18(7): p. 1479-86.

20. White, P.S., et al., A region of consistent deletion in neuroblastoma maps within human chromosome 1p36.2-36.3. Proc Natl Acad Sci U S A, 1995. 92(12): p. 5520-4.

21. Bown, N., et al., Gain of chromosome arm $17 q$ and adverse outcome in patients with neuroblastoma. The New England journal of medicine, 1999. 340(25): $\mathrm{p}$. 1954-61.

22. Alberts, B.J., A.; Lewis, J.; Raff, M.; Roberts, K.; Walter, P.;, Molecular biology of the cell. Fourth ed, ed. S. Gibbs. 2002, New York: Garland Science.

23. Weinberg, R.A., The biology of cancer, ed. E. Zayatz. 2007, New York: Garland Science.

24. Lockshin, R.A. and C.M. Williams, Programmed Cell Death .2. Endocrine Potentiation of the Breakdown of the Intersegmental Muscles of Silkmoths. Journal of Insect Physiology, 1964. 10(4): p. 643-649.

25. Kroemer, G., et al., Classification of cell death: recommendations of the Nomenclature Committee on Cell Death 2009. Cell Death Differ, 2009. 16(1): p. 3-11.

26. Vandenabeele, P., et al., Molecular mechanisms of necroptosis: an ordered cellular explosion. Nature reviews. Molecular cell biology, 2010. 11(10): p. 70014.

27. Yuan, J. and G. Kroemer, Alternative cell death mechanisms in development and beyond. Genes Dev, 2010. 24(23): p. 2592-602.

28. Pop, C. and G.S. Salvesen, Human caspases: activation, specificity, and regulation. The Journal of biological chemistry, 2009. 284(33): p. 21777-81.

29. Ahmad, M., et al., Identification and characterization of murine caspase-14, a new member of the caspase family. Cancer Res, 1998. 58(22): p. 5201-5.

30. Denault, J.B. and G.S. Salvesen, Caspases. Current protocols in protein science / editorial board, John E. Coligan ... [et al.], 2002. Chapter 21: p. Unit 218.

31. Shi, Y., Caspase activation: revisiting the induced proximity model. Cell, 2004. 117(7): p. 855-8.

32. Grenet, J., et al., Structure and chromosome localization of the human CASP8 gene. Gene, 1999. 226(2): p. 225-32.

33. Kischkel, F.C., et al., Assignment of CASP8 to human chromosome band $2 q 33$-> $q 34$ and Casp 8 to the murine syntenic region on chromosome 1B-proximal C by in situ hybridization. Cytogenetics and Cell Genetics, 1998. 82(1-2): p. 95-96.

34. Muzio, M., et al., FLICE, a novel FADD-homologous ICE/CED-3-like protease, is recruited to the CD95 (Fas/APO-1) death--inducing signaling complex. Cell, 1996. 85(6): p. 817-27. 
35. Chinnaiyan, A.M., et al., FADD, a novel death domain-containing protein, interacts with the death domain of Fas and initiates apoptosis. Cell, 1995. 81(4): p. 505-12.

36. Nicholson, D.W., Caspase structure, proteolytic substrates, and function during apoptotic cell death. Cell death and differentiation, 1999. 6(11): p. 1028-42.

37. Wilson, K.P., et al., Structure and mechanism of interleukin-1 beta converting enzyme. Nature, 1994. 370(6487): p. 270-5.

38. Walker, N.P., et al., Crystal structure of the cysteine protease interleukin-1 betaconverting enzyme: a (p20/p10)2 homodimer. Cell, 1994. 78(2): p. 343-52.

39. Muzio, M., et al., An induced proximity model for caspase-8 activation. J Biol Chem, 1998. 273(5): p. 2926-30.

40. Salvesen, G.S. and S.J. Riedl, Structure of the Fas/FADD complex: a conditional death domain complex mediating signaling by receptor clustering. Cell Cycle, 2009. 8(17): p. 2723-7.

41. Rupinder, S.K., A.K. Gurpreet, and S. Manjeet, Cell suicide and caspases. Vascul Pharmacol, 2007. 46(6): p. 383-93.

42. Yu, L., et al., Regulation of an ATG7-beclin 1 program of autophagic cell death by caspase-8. Science, 2004. 304(5676): p. 1500-2.

43. Oral, O., et al., Cleavage of Atg3 protein by caspase-8 regulates autophagy during receptor-activated cell death. Apoptosis, 2012. 17(8): p. 810-20.

44. Li, H., et al., Cleaving Beclin 1 to suppress autophagy in chemotherapy-induced apoptosis. Autophagy, 2011. 7(10): p. 1239-41.

45. Hou, W., et al., Autophagic degradation of active caspase-8: a crosstalk mechanism between autophagy and apoptosis. Autophagy, 2010. 6(7): p. 891900.

46. Csomos, R.A., G.F. Brady, and C.S. Duckett, Enhanced cytoprotective effects of the inhibitor of apoptosis protein cellular IAP1 through stabilization with TRAF2. J Biol Chem, 2009. 284(31): p. 20531-9.

47. Micheau, O. and J. Tschopp, Induction of TNF receptor I-mediated apoptosis via two sequential signaling complexes. Cell, 2003. 114(2): p. 181-90.

48. Wang, L., F. Du, and X. Wang, TNF-alpha induces two distinct caspase-8 activation pathways. Cell, 2008. 133(4): p. 693-703.

49. Christofferson, D.E. and J. Yuan, Necroptosis as an alternative form of programmed cell death. Current opinion in cell biology, 2010. 22(2): p. 263-8.

50. Reubold, T.F. and S. Eschenburg, A molecular view on signal transduction by the apoptosome. Cell Signal, 2012. 24(7): p. 1420-5.

51. Hengartner, M.O., The biochemistry of apoptosis. Nature, 2000. 407(6805): p. 770-6.

52. Boldin, M.P., et al., A novel protein that interacts with the death domain of Fas/APOI contains a sequence motif related to the death domain. J Biol Chem, 1995. 270(14): p. 7795-8.

53. Kischkel, F.C., et al., Cytotoxicity-dependent APO-1 (Fas/CD95)-associated proteins form a death-inducing signaling complex (DISC) with the receptor. EMBO J, 1995. 14(22): p. 5579-88. 
54. Kang, T.B., et al., Mutation of a self-processing site in caspase-8 compromises its apoptotic but not its nonapoptotic functions in bacterial artificial chromosometransgenic mice. Journal of immunology, 2008. 181(4): p. 2522-32.

55. Pop, C., et al., Role of proteolysis in caspase-8 activation and stabilization. Biochemistry, 2007. 46(14): p. 4398-407.

56. Oberst, A., et al., Inducible dimerization and inducible cleavage reveal a requirement for both processes in caspase-8 activation. The Journal of biological chemistry, 2010. 285(22): p. 16632-42.

57. Boatright, K.M. and G.S. Salvesen, Mechanisms of caspase activation. Current opinion in cell biology, 2003. 15(6): p. 725-31.

58. Boatright, K.M., et al., A unified model for apical caspase activation. Mol Cell, 2003. 11(2): p. 529-41.

59. Jin, Z., et al., Cullin3-based polyubiquitination and p62-dependent aggregation of caspase-8 mediate extrinsic apoptosis signaling. Cell, 2009. 137(4): p. 721-35.

60. Scaffidi, C., et al., Two CD95 (APO-1/Fas) signaling pathways. The EMBO journal, 1998. 17(6): p. 1675-87.

61. Algeciras-Schimnich, A., et al., Two CD95 tumor classes with different sensitivities to antitumor drugs. Proc Natl Acad Sci U S A, 2003. 100(20): p. 11445-50.

62. Lazebnik, Y.A., et al., Cleavage of poly(ADP-ribose) polymerase by a proteinase with properties like ICE. Nature, 1994. 371(6495): p. 346-7.

63. Samraj, A.K., et al., Loss of caspase-9 provides genetic evidence for the type I/II concept of CD95-mediated apoptosis. The Journal of biological chemistry, 2006. 281(40): p. 29652-9.

64. Thome, M., et al., Viral FLICE-inhibitory proteins (FLIPs) prevent apoptosis induced by death receptors. Nature, 1997. 386(6624): p. 517-21.

65. Hu, S., et al., A novel family of viral death effector domain-containing molecules that inhibit both CD-95-and tumor necrosis factor receptor-1-induced apoptosis. J Biol Chem, 1997. 272(15): p. 9621-4.

66. Irmler, M., et al., Inhibition of death receptor signals by cellular FLIP. Nature, 1997. 388(6638): p. 190-5.

67. Goltsev, Y.V., et al., CASH, a novel caspase homologue with death effector domains. J Biol Chem, 1997. 272(32): p. 19641-4.

68. Shu, H.B., D.R. Halpin, and D.V. Goeddel, Casper is a FADD-and caspaserelated inducer of apoptosis. Immunity, 1997. 6(6): p. 751-63.

69. Inohara, N., et al., CLARP, a death effector domain-containing protein interacts with caspase-8 and regulates apoptosis. Proc Natl Acad Sci U S A, 1997. 94(20): p. 10717-22.

70. Micheau, O., et al., The long form of FLIP is an activator of caspase-8 at the Fas death-inducing signaling complex. J Biol Chem, 2002. 277(47): p. 45162-71.

71. Scaffidi, C., et al., The role of c-FLIP in modulation of CD95-induced apoptosis. J Biol Chem, 1999. 274(3): p. 1541-8.

72. Kataoka, T. and J. Tschopp, N-terminal fragment of $c-F L I P(L)$ processed by caspase 8 specifically interacts with TRAF2 and induces activation of the NFkappaB signaling pathway. Mol Cell Biol, 2004. 24(7): p. 2627-36. 
73. Oberst, A., et al., Catalytic activity of the caspase-8-FLIP(L) complex inhibits RIPK3-dependent necrosis. Nature, 2011. 471(7338): p. 363-7.

74. Hughes, M.A., et al., Reconstitution of the death-inducing signaling complex reveals a substrate switch that determines CD95-mediated death or survival. Molecular cell, 2009. 35(3): p. 265-79.

75. Scott, F.L., et al., Caspase-8 cleaves histone deacetylase 7 and abolishes its transcription repressor function. J Biol Chem, 2008. 283(28): p. 19499-510.

76. Pop, C., et al., FLIP $(L)$ induces caspase 8 activity in the absence of interdomain caspase 8 cleavage and alters substrate specificity. Biochem J, 2011. 433(3): p. 447-57.

77. Bagnoli, M., S. Canevari, and D. Mezzanzanica, Cellular FLICE-inhibitory protein (c-FLIP) signalling: a key regulator of receptor-mediated apoptosis in physiologic context and in cancer. Int J Biochem Cell Biol, 2010. 42(2): p. 210-3.

78. Yu, J.W., P.D. Jeffrey, and Y. Shi, Mechanism of procaspase-8 activation by cFLIPL. Proc Natl Acad Sci U S A, 2009. 106(20): p. 8169-74.

79. Ben Moshe, T., et al., Role of caspase- 8 in hepatocyte response to infection and injury in mice. Hepatology, 2007. 45(4): p. 1014-24.

80. Pellegrini, M., et al., FADD and caspase-8 are required for cytokine-induced proliferation of hemopoietic progenitor cells. Blood, 2005. 106(5): p. 1581-9.

81. Gilot, D., et al., A role for caspase-8 and c-FLIPL in proliferation and cell-cycle progression of primary hepatocytes. Carcinogenesis, 2005. 26(12): p. 2086-94.

82. Kang, T.B., et al., Caspase-8 serves both apoptotic and nonapoptotic roles. J Immunol, 2004. 173(5): p. 2976-84.

83. Salmena, L., et al., Essential role for caspase 8 in T-cell homeostasis and T-cellmediated immunity. Genes Dev, 2003. 17(7): p. 883-95.

84. Salmena, L. and R. Hakem, Caspase-8 deficiency in T cells leads to a lethal lymphoinfiltrative immune disorder. The Journal of experimental medicine, 2005. 202(6): p. 727-32.

85. Chun, H.J., et al., Pleiotropic defects in lymphocyte activation caused by caspase8 mutations lead to human immunodeficiency. Nature, 2002. 419(6905): p. 395-9.

86. Arechiga, A.F., et al., A Fas-associated death domain protein/caspase-8-signaling axis promotes $S$-phase entry and maintains $S 6$ kinase activity in $T$ cells responding to $I L-2$. Journal of immunology, 2007. 179(8): p. 5291-300.

87. Finlay, D., A. Howes, and K. Vuori, Critical role for caspase-8 in epidermal growth factor signaling. Cancer research, 2009. 69(12): p. 5023-9.

88. Barbero, S., et al., Caspase-8 association with the focal adhesion complex promotes tumor cell migration and metastasis. Cancer research, 2009. 69(9): p. 3755-63.

89. Helfer, B., et al., Caspase-8 promotes cell motility and calpain activity under nonapoptotic conditions. Cancer research, 2006. 66(8): p. 4273-8.

90. Frisch, S.M., Caspase-8: fly or die. Cancer research, 2008. 68(12): p. 4491-3.

91. Torres, V.A., et al., Rab5 mediates caspase-8-promoted cell motility and metastasis. Molecular biology of the cell, 2010. 21(2): p. 369-76.

92. Chaudhary, P.M., et al., Activation of the NF-kappaB pathway by caspase 8 and its homologs. Oncogene, 2000. 19(39): p. 4451-60. 
93. Hu, W.H., H. Johnson, and H.B. Shu, Activation of NF-kappaB by FADD, Casper, and caspase-8. The Journal of biological chemistry, 2000. 275(15): p. 10838-44.

94. Tibbetts, M.D., L. Zheng, and M.J. Lenardo, The death effector domain protein family: regulators of cellular homeostasis. Nature immunology, 2003. 4(5): p. 404-9.

95. Su, H., et al., Requirement for caspase-8 in NF-kappaB activation by antigen receptor. Science, 2005. 307(5714): p. 1465-8.

96. Bidere, N., et al., Caspase-8 regulation by direct interaction with TRAF6 in T cell receptor-induced NF-kappaB activation. Current biology : CB, 2006. 16(16): p. 1666-71.

97. Ehrenschwender, M., et al., Mutant PIK3CA licenses TRAIL and CD95L to induce non-apoptotic caspase-8-mediated ROCK activation. Cell death and differentiation, 2010. 17(9): p. 1435-47.

98. Lee, P., et al., Dynamic expression of epidermal caspase 8 simulates a wound healing response. Nature, 2009. 458(7237): p. 519-23.

99. Wertz, I.E. and V.M. Dixit, Regulation of death receptor signaling by the ubiquitin system. Cell Death Differ, 2010. 17(1): p. 14-24.

100. van Raam, B.J. and G.S. Salvesen, Proliferative versus apoptotic functions of caspase-8 Hetero or homo: the caspase-8 dimer controls cell fate. Biochim Biophys Acta, 2012. 1824(1): p. 113-22.

101. Cursi, S., et al., Src kinase phosphorylates Caspase-8 on Tyr380: a novel mechanism of apoptosis suppression. The EMBO journal, 2006. 25(9): p. 1895905.

102. Barbero, S., et al., Identification of a critical tyrosine residue in caspase 8 that promotes cell migration. The Journal of biological chemistry, 2008. 283(19): p. 13031-4.

103. Matthess, Y., et al., Cdk1/cyclin B1 controls Fas-mediated apoptosis by regulating caspase-8 activity. Molecular and cellular biology, 2010. 30(24): p. 5726-40.

104. Peng, C., et al., Phosphorylation of caspase-8 (Thr-263) by ribosomal S6 kinase 2 (RSK2) mediates caspase-8 ubiquitination and stability. J Biol Chem, 2011. 286(9): p. 6946-54.

105. Alvarado-Kristensson, M., et al., p38-MAPK signals survival by phosphorylation of caspase-8 and caspase-3 in human neutrophils. The Journal of experimental medicine, 2004. 199(4): p. 449-58.

106. Matsuoka, S., et al., ATM and ATR substrate analysis reveals extensive protein networks responsive to DNA damage. Science, 2007. 316(5828): p. 1160-6.

107. Kurokawa, M. and S. Kornbluth, Caspases and kinases in a death grip. Cell, 2009. 138(5): p. 838-54.

108. Sanchez, I., et al., Caspase-8 is required for cell death induced by expanded polyglutamine repeats. Neuron, 1999. 22(3): p. 623-33.

109. Qin, Z.H., et al., Pro-caspase-8 is predominantly localized in mitochondria and released into cytoplasm upon apoptotic stimulation. The Journal of biological chemistry, 2001. 276(11): p. 8079-86. 
110. Benchoua, A., et al., Active caspase-8 translocates into the nucleus of apoptotic cells to inactivate poly(ADP-ribose) polymerase-2. J Biol Chem, 2002. 277(37): p. 34217-22.

111. Mielgo, A., et al., Paclitaxel promotes a caspase 8-mediated apoptosis through death effector domain association with microtubules. Oncogene, 2009. 28(40): p. 3551-3562.

112. Mielgo, A., et al., The Death Effector Domains of Caspase-8 Induce Terminal Differentiation. PLoS One, 2009. 4(11).

113. Besnault-Mascard, L., et al., Caspase-8 sumoylation is associated with nuclear localization. Oncogene, 2005. 24(20): p. 3268-73.

114. Kaufmann, S.H. and D.L. Vaux, Alterations in the apoptotic machinery and their potential role in anticancer drug resistance. Oncogene, 2003. 22(47): p. 7414-30.

115. Grau, E., et al., Hypermethylation of apoptotic genes as independent prognostic factor in neuroblastoma disease. Mol Carcinog, 2011. 50(3): p. 153-62.

116. Fulda, S., et al., Sensitization for death receptor-or drug-induced apoptosis by reexpression of caspase-8 through demethylation or gene transfer. Oncogene, 2001. 20(41): p. 5865-77.

117. Kamimatsuse, A., et al., Detection of $C p G$ island hypermethylation of caspase-8 in neuroblastoma using an oligonucleotide array. Pediatric blood \& cancer, 2009. 52(7): p. 777-83.

118. Harada, K., et al., Deregulation of caspase 8 and 10 expression in pediatric tumors and cell lines. Cancer research, 2002. 62(20): p. 5897-901.

119. Hopkins-Donaldson, S., et al., Silencing of death receptor and caspase-8 expression in small cell lung carcinoma cell lines and tumors by DNA methylation. Cell death and differentiation, 2003. 10(3): p. 356-64.

120. Kim, H.S., et al., Inactivating mutations of caspase-8 gene in colorectal carcinomas. Gastroenterology, 2003. 125(3): p. 708-15.

121. Gonzalez-Gomez, P., et al., Deletion and aberrant $\mathrm{Cp} G$ island methylation of Caspase 8 gene in medulloblastoma. Oncology reports, 2004. 12(3): p. 663-6.

122. Soung, Y.H., et al., CASPASE-8 gene is inactivated by somatic mutations in gastric carcinomas. Cancer Res, 2005. 65(3): p. 815-21.

123. Martinez, R., et al., CpG island promoter hypermethylation of the pro-apoptotic gene caspase-8 is a common hallmark of relapsed glioblastoma multiforme. Carcinogenesis, 2007. 28(6): p. 1264-8.

124. Pingoud-Meier, C., et al., Loss of caspase-8 protein expression correlates with unfavorable survival outcome in childhood medulloblastoma. Clinical cancer research : an official journal of the American Association for Cancer Research, 2003. 9(17): p. 6401-9.

125. Yang, Q., et al., Methylation of CASP8, DCR2, and HIN-1 in neuroblastoma is associated with poor outcome. Clinical cancer research : an official journal of the American Association for Cancer Research, 2007. 13(11): p. 3191-7.

126. Teitz, T., J.M. Lahti, and V.J. Kidd, Aggressive childhood neuroblastomas do not express caspase-8: an important component of programmed cell death. Journal of molecular medicine, 2001. 79(8): p. 428-36. 
127. Jiang, M., et al., Retinoic acid induces caspase-8 transcription via phospho-CREB and increases apoptotic responses to death stimuli in neuroblastoma cells. Biochimica et biophysica acta, 2008. 1783(6): p. 1055-67.

128. Tekautz, T.M., et al., Evaluation of IFN-gamma effects on apoptosis and gene expression in neuroblastoma--preclinical studies. Biochim Biophys Acta, 2006. 1763(10): p. 1000-10.

129. Johnsen, J.I., et al., Synergistic induction of apoptosis in neuroblastoma cells using a combination of cytostatic drugs with interferon-gamma and TRAIL. Int $\mathrm{J}$ Oncol, 2004. 25(6): p. 1849-57.

130. Fulda, S. and K.M. Debatin, 5-Aza-2'-deoxycytidine and IFN-gamma cooperate to sensitize for TRAIL-induced apoptosis by upregulating caspase-8. Oncogene, 2006. 25(37): p. 5125-33.

131. Soung, Y.H., et al., Caspase-8 gene is frequently inactivated by the frameshift somatic mutation 1225_1226delTG in hepatocellular carcinomas. Oncogene, 2005. 24(1): p. 141-7.

132. Strater, J., et al., Expression and prognostic significance of APAF-1, caspase-8 and caspase-9 in stage II/III colon carcinoma: caspase-8 and caspase-9 is associated with poor prognosis. Int J Cancer, 2010. 127(4): p. 873-80.

133. Elrod, H.A., et al., Analysis of death receptor 5 and caspase-8 expression in primary and metastatic head and neck squamous cell carcinoma and their prognostic impact. PLoS One, 2010. 5(8): p. e12178.

134. Hopkins-Donaldson, S., et al., Loss of caspase-8 expression in highly malignant human neuroblastoma cells correlates with resistance to tumor necrosis factorrelated apoptosis-inducing ligand-induced apoptosis. Cancer Res, 2000. 60(16): p. 4315-9.

135. Grotzer, M.A., et al., Resistance to TRAIL-induced apoptosis in primitive neuroectodermal brain tumor cells correlates with a loss of caspase-8 expression. Oncogene, 2000. 19(40): p. 4604-10.

136. Eggert, A., et al., Resistance to tumor necrosis factor-related apoptosis-inducing ligand (TRAIL)-induced apoptosis in neuroblastoma cells correlates with a loss of caspase-8 expression. Cancer Res, 2001. 61(4): p. 1314-9.

137. Yang, X., et al., Induction of caspase 8 by interferon gamma renders some neuroblastoma $(N B)$ cells sensitive to tumor necrosis factor-related apoptosisinducing ligand (TRAIL) but reveals that a lack of membrane TR1/TR2 also contributes to TRAIL resistance in NB. Cancer Res, 2003. 63(5): p. 1122-9.

138. Casciano, I., et al., Expression of the caspase-8 gene in neuroblastoma cells is regulated through an essential interferon-sensitive response element (ISRE). Cell Death Differ, 2004. 11(1): p. 131-4.

139. Merchant, M.S., et al., Interferon gamma enhances the effectiveness of tumor necrosis factor-related apoptosis-inducing ligand receptor agonists in a xenograft model of Ewing's sarcoma. Cancer Res, 2004. 64(22): p. 8349-56.

140. Ruiz-Ruiz, C., et al., The up-regulation of human caspase-8 by interferon-gamma in breast tumor cells requires the induction and action of the transcription factor interferon regulatory factor-1. J Biol Chem, 2004. 279(19): p. 19712-20.

141. Stupack, D.G., Caspase-8 as a therapeutic target in cancer. Cancer Lett, 2013. 332(2): p. 133-40. 
142. Fulda, S., Caspase-8 in cancer biology and therapy. Cancer Lett, 2009. 281(2): p. 128-33.

143. Fulda, S., et al., Loss of caspase-8 expression does not correlate with MYCN amplification, aggressive disease, or prognosis in neuroblastoma. Cancer Res, 2006. 66(20): p. 10016-23.

144. Takita, J., et al., Absent or reduced expression of the caspase 8 gene occurs frequently in neuroblastoma, but not commonly in Ewing sarcoma or rhabdomyosarcoma. Med Pediatr Oncol, 2000. 35(6): p. 541-3.

145. Stupack, D.G., et al., Potentiation of neuroblastoma metastasis by loss of caspase-8. Nature, 2006. 439(7072): p. 95-9.

146. Finlay, D. and K. Vuori, Novel noncatalytic role for caspase-8 in promoting SRCmediated adhesion and Erk signaling in neuroblastoma cells. Cancer research, 2007. 67(24): p. 11704-11.

147. Alam, A., et al., Early activation of caspases during T lymphocyte stimulation results in selective substrate cleavage in nonapoptotic cells. The Journal of experimental medicine, 1999. 190(12): p. 1879-90.

148. Jean, C., et al., Influence of stress on extracellular matrix and integrin biology. Oncogene, 2011. 30(24): p. 2697-706.

149. Senft, J., B. Helfer, and S.M. Frisch, Caspase-8 interacts with the $p 85$ subunit of phosphatidylinositol 3-kinase to regulate cell adhesion and motility. Cancer research, 2007. 67(24): p. 11505-9.

150. Gelman, D.M., et al., Transgenic mice engineered to target Cre/loxP-mediated DNA recombination into catecholaminergic neurons. Genesis, 2003. 36(4): p. 196-202.

151. Weiss, W.A., et al., Targeted expression of MYCN causes neuroblastoma in transgenic mice. EMBO J, 1997. 16(11): p. 2985-95.

152. Teitz, T., et al., Th-MYCN mice with caspase-8 deficiency develop advanced neuroblastoma with bone marrow metastasis. Cancer Res, 2013.

153. George, R.E., et al., Phase I study of decitabine with doxorubicin and cyclophosphamide in children with neuroblastoma and other solid tumors: a Children's Oncology Group study. Pediatric blood \& cancer, 2010. 55(4): p. 62938.

154. Matthay, K.K., Neuroblastoma: a clinical challenge and biologic puzzle. CA: a cancer journal for clinicians, 1995. 45(3): p. 179-92.

155. Johnsen, J.I., et al., Embryonal neural tumours and cell death. Apoptosis : an international journal on programmed cell death, 2009. 14(4): p. 424-38.

156. Teitz, T., D.G. Stupack, and J.M. Lahti, Halting neuroblastoma metastasis by controlling integrin-mediated death. Cell cycle, 2006. 5(7): p. 681-5.

157. Hughes, M.A., et al., Reconstitution of the death-inducing signaling complex reveals a substrate switch that determines CD95-mediated death or survival. Mol Cell, 2009. 35(3): p. 265-79.

158. Scaffidi, C., et al., Two CD95 (APO-1/Fas) signaling pathways. EMBO J, 1998. 17(6): p. 1675-87.

159. Schmitz, I., et al., Differences between CD95 type I and II cells detected with the CD95 ligand. Cell death and differentiation, 1999. 6(9): p. 821-2. 
160. Sordet, O., et al., Specific involvement of caspases in the differentiation of monocytes into macrophages. Blood, 2002. 100(13): p. 4446-53.

161. Mogi, M. and A. Togari, Activation of caspases is required for osteoblastic differentiation. The Journal of biological chemistry, 2003. 278(48): p. 47477-82.

162. Kennedy, N.J., et al., Caspase activation is required for $T$ cell proliferation. The Journal of experimental medicine, 1999. 190(12): p. 1891-6.

163. Kang, T.B., et al., Mutation of a self-processing site in caspase-8 compromises its apoptotic but not its nonapoptotic functions in bacterial artificial chromosometransgenic mice. J Immunol, 2008. 181(4): p. 2522-32.

164. Scaffidi, C., et al., FLICE is predominantly expressed as two functionally active isoforms, caspase-8/a and caspase-8/b. J Biol Chem, 1997. 272(43): p. 26953-8.

165. Teitz, T., et al., Preclinical models for neuroblastoma: establishing a baseline for treatment. PLoS One, 2011. 6(4): p. e19133.

166. Clark, E.A., et al., Genomic analysis of metastasis reveals an essential role for RhoC. Nature, 2000. 406(6795): p. 532-5.

167. Leverrier, S., G.S. Salvesen, and C.M. Walsh, Enzymatically active single chain caspase-8 maintains T-cell survival during clonal expansion. Cell Death Differ, 2011. 18(1): p. 90-8.

168. Zuzak, T.J., et al., Loss of caspase- $8 \mathrm{mRNA}$ expression is common in childhood primitive neuroectodermal brain tumour/medulloblastoma. Eur J Cancer, 2002. 38(1): p. 83-91.

169. Shivapurkar, N., et al., Differential inactivation of caspase-8 in lung cancers. Cancer Biol Ther, 2002. 1(1): p. 65-9.

170. Knighton, D.R., V.D. Fiegel, and G.D. Phillips, The assay of angiogenesis. Prog Clin Biol Res, 1991. 365: p. 291-9.

171. Jakob, W., et al., The chick embryo choriallantoic membrane as a bioassay for angiogenesis factors: reactions induced by carrier materials. Exp Pathol (Jena), 1978. 15(5): p. 241-9.

172. Persons, D.A., et al., Enforced expression of the GATA-2 transcription factor blocks normal hematopoiesis. Blood, 1999. 93(2): p. 488-99.

173. Yamamoto, K.N., et al., Characterization of environmental chemicals with potential for DNA damage using isogenic DNA repair-deficient chicken DT40 cell lines. Environ Mol Mutagen, 2011. 52(7): p. 547-61.

174. Perera, F., et al., In utero DNA damage from environmental pollution is associated with somatic gene mutation in newborns. Cancer Epidemiol Biomarkers Prev, 2002. 11(10 Pt 1): p. 1134-7.

175. Savitsky, K., et al., The complete sequence of the coding region of the ATM gene reveals similarity to cell cycle regulators in different species. Human molecular genetics, 1995. 4(11): p. 2025-32.

176. Lavin, M.F., Ataxia-telangiectasia: from a rare disorder to a paradigm for cell signalling and cancer. Nature reviews. Molecular cell biology, 2008. 9(10): p. 759-69.

177. McKinnon, P.J., ATM and ataxia telangiectasia. EMBO reports, 2004. 5(8): p. 772-6.

178. Perry, J.J. and J.A. Tainer, All Stressed Out Without ATM Kinase. Science signaling, 2011. 4(167): p. pe18. 
179. McKinnon, P.J., Ataxia telangiectasia: new neurons and ATM. Trends in molecular medicine, 2001. 7(6): p. 233-4.

180. Lee, J.H. and T.T. Paull, Activation and regulation of ATM kinase activity in response to DNA double-strand breaks. Oncogene, 2007. 26(56): p. 7741-8.

181. Williams, G.J., S.P. Lees-Miller, and J.A. Tainer, Mre11-Rad50-Nbs 1 conformations and the control of sensing, signaling, and effector responses at DNA double-strand breaks. DNA Repair (Amst), 2010. 9(12): p. 1299-306.

182. Stracker, T.H. and J.H. Petrini, The MRE11 complex: starting from the ends. Nature reviews. Molecular cell biology, 2011. 12(2): p. 90-103.

183. Hopfner, K.P., et al., The Rad50 zinc-hook is a structure joining Mrel1 complexes in DNA recombination and repair. Nature, 2002. 418(6897): p. 562-6.

184. Moreno-Herrero, F., et al., Mesoscale conformational changes in the DNA-repair complex Rad50/Mre11/Nbs1 upon binding DNA. Nature, 2005. 437(7057): p. 440-3.

185. Falck, J., J. Coates, and S.P. Jackson, Conserved modes of recruitment of ATM, ATR and DNA-PKcs to sites of DNA damage. Nature, 2005. 434(7033): p. 605-11.

186. Williams, R.S., et al., Nbs1 flexibly tethers Ctp1 and Mre11-Rad50 to coordinate DNA double-strand break processing and repair. Cell, 2009. 139(1): p. 87-99.

187. Bakkenist, C.J. and M.B. Kastan, DNA damage activates ATM through intermolecular autophosphorylation and dimer dissociation. Nature, 2003. 421(6922): p. 499-506.

188. McKinnon, P.J., ATM and the molecular pathogenesis of ataxia telangiectasia. Annu Rev Pathol, 2012. 7: p. 303-21.

189. Ushio-Fukai, M. and Y. Nakamura, Reactive oxygen species and angiogenesis: NADPH oxidase as target for cancer therapy. Cancer letters, 2008. 266(1): p. 3752.

190. Lee, J.K., et al., NADPH oxidase promotes pancreatic cancer cell survival via inhibiting JAK2 dephosphorylation by tyrosine phosphatases. Gastroenterology, 2007. 133(5): p. 1637-48.

191. Du, J., et al., Role of Rac1-dependent NADPH oxidase in the growth of pancreatic cancer. Cancer gene therapy, 2011. 18(2): p. 135-43.

192. Wu, Z.H., et al., Molecular linkage between the kinase ATM and NF-kappaB signaling in response to genotoxic stimuli. Science, 2006. 311(5764): p. 1141-6.

193. Huang, T.T., et al., Sequential modification of NEMO/IKKgamma by SUMO-1 and ubiquitin mediates NF-kappaB activation by genotoxic stress. Cell, 2003. 115(5): p. 565-76.

194. Biton, S. and A. Ashkenazi, NEMO and RIP1 control cell fate in response to extensive DNA damage via TNF-alpha feedforward signaling. Cell, 2011. 145(1): p. 92-103.

195. Bredemeyer, A.L., et al., DNA double-strand breaks activate a multi-functional genetic program in developing lymphocytes. Nature, 2008. 456(7223): p. 819-23.

196. Eaton, J.S., et al., Ataxia-telangiectasia mutated kinase regulates ribonucleotide reductase and mitochondrial homeostasis. J Clin Invest, 2007. 117(9): p. 272334. 
197. Ambrose, M., J.V. Goldstine, and R.A. Gatti, Intrinsic mitochondrial dysfunction in ATM-deficient lymphoblastoid cells. Human molecular genetics, 2007. 16(18): p. 2154-64.

198. Kim, W.S., et al., Analysis of apolipoprotein E nuclear localization using green fluorescent protein and biotinylation approaches. Biochem J, 2008. 409(3): p. 701-9.

199. Yang, D.Q. and M.B. Kastan, Participation of ATM in insulin signalling through phosphorylation of eIF-4E-binding protein 1. Nature cell biology, 2000. 2(12): $\mathrm{p}$. 893-8.

200. Shen, Y. and E. White, p53-dependent apoptosis pathways. Adv Cancer Res, 2001. 82: p. $55-84$.

201. Stagni, V., et al., ATM kinase activity modulates cFLIP protein levels: potential interplay between DNA damage signalling and TRAIL-induced apoptosis. Carcinogenesis, 2010. 31(11): p. 1956-63.

202. Kamer, I., et al., Proapoptotic BID is an ATM effector in the DNA-damage response. Cell, 2005. 122(4): p. 593-603.

203. Unsal-Kacmaz, K., et al., Preferential binding of ATR protein to UV-damaged DNA. Proc Natl Acad Sci U S A, 2002. 99(10): p. 6673-8.

204. Finlay, D., et al., Novel HTS strategy identifies TRAIL-sensitizing compounds acting specifically through the caspase-8 apoptotic axis. PLoS One, 2010. 5(10): p. e13375.

205. Weibrecht, I., et al., Proximity ligation assays: a recent addition to the proteomics toolbox. Expert Rev Proteomics, 2010. 7(3): p. 401-9. 


\section{APPENDIX. CHAPTER 3 SUPPLEMENTAL FIGURES}

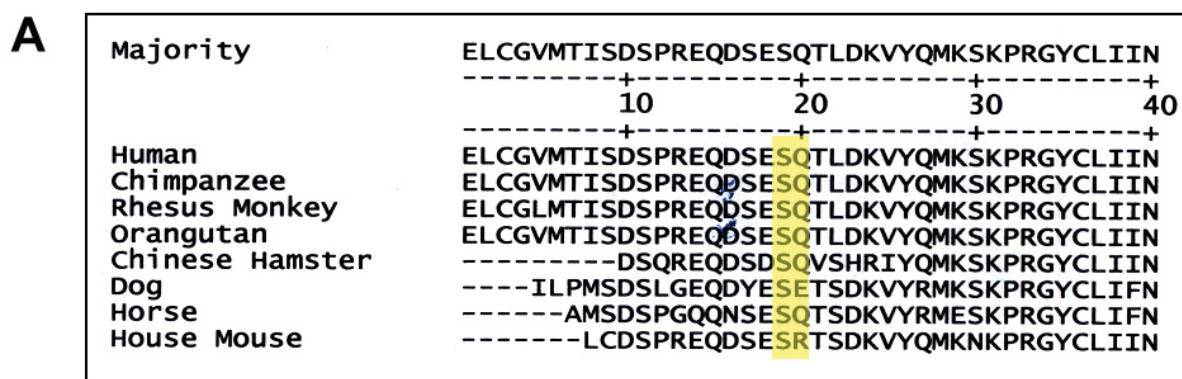

B

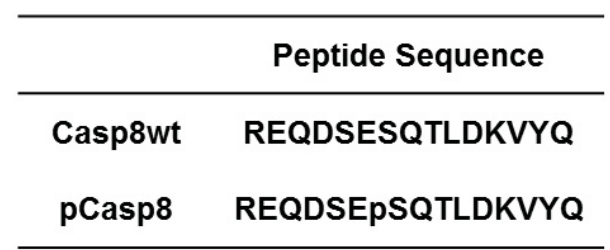

C

\begin{tabular}{cc}
\hline & Epitope Sequence \\
\hline BID & EADSESQEDII \\
Casp8 & EQDSESQTLDK \\
\hline
\end{tabular}

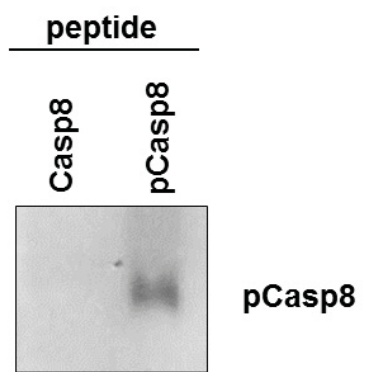

Figure A-1. Serine 219 of caspase-8 is highly conserved.

(A) Comparison of the S219 residue of human casp8 to other species. (B) Peptide sequence and western blot analysis of custom peptides. (C) Comparison of the BID S78 to the S219 epitopes of casp8. 


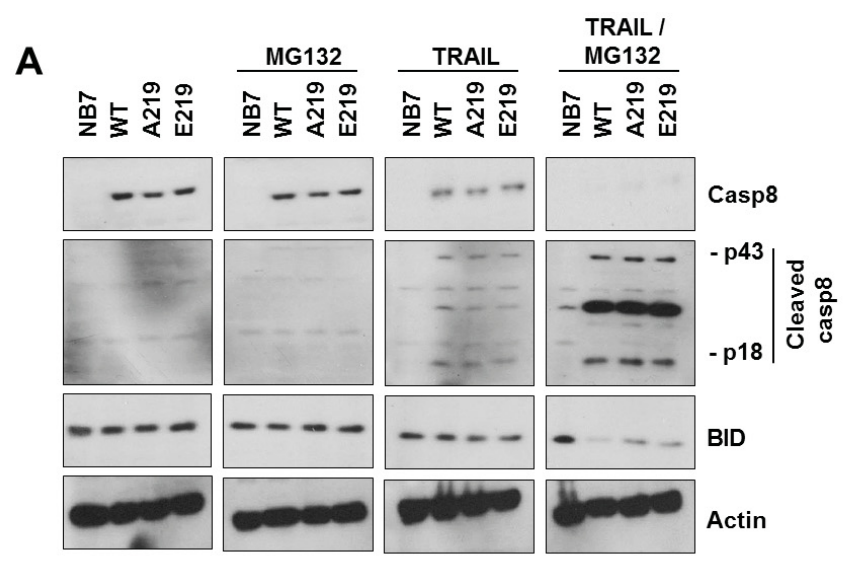

B

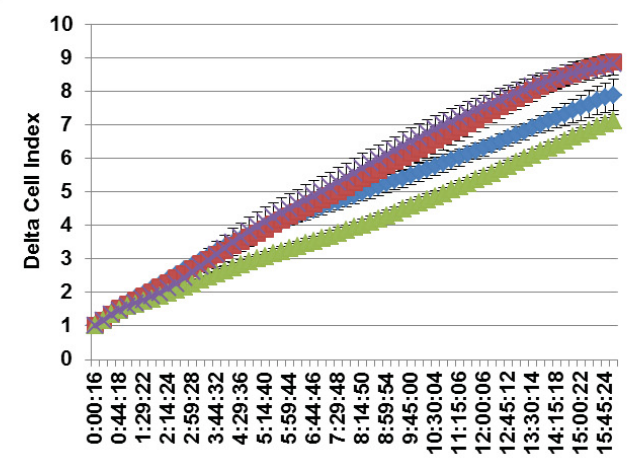

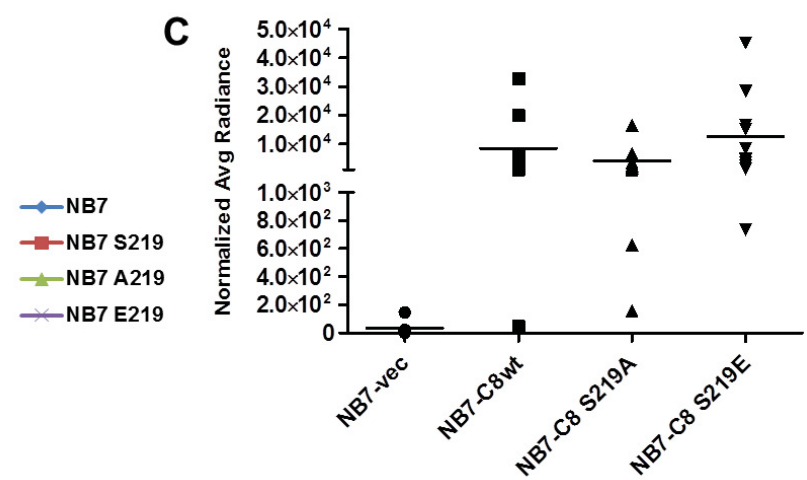

Figure A-2. Caspase-8 phosphorylation in apoptosis, adhesion and tumor growth. (A) NB7 cells deficient, expressing wt or a mutant construct of casp8 were exposed to MG132 [1 $\mu \mathrm{M}$ ], TRAIL [50 ng/mL], or both for 24 hours and blotted for cleaved casp8 or downstream effects of active casp8, cleaved BID. (B) Adhesion assay performed in real-time to collagen coated plates $[10 \mu \mathrm{g} / \mathrm{mL}]$. Assay performed using the xCELLigence system. (C) NB7 cells expressing the indicated casp8 constructs were injected into the flank of nude mice at a concentration of $2 \times 10^{5}$ cells/mouse and analyzed by bioluminescence weekly, data shown here at the seven week time point. Error bars, \pm s.e.m. 
A
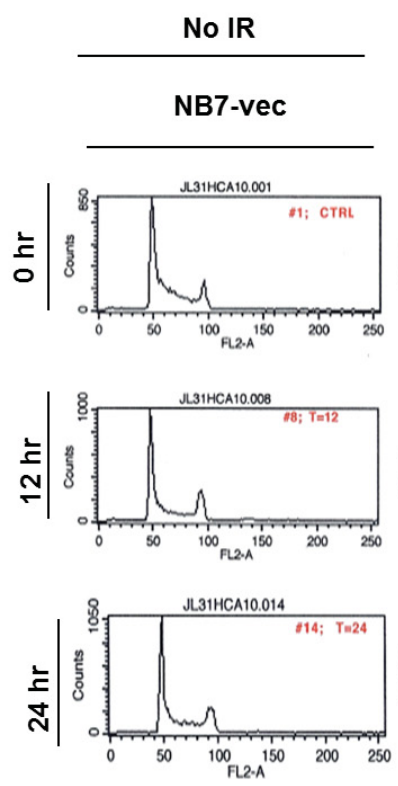

IR 10 Gy
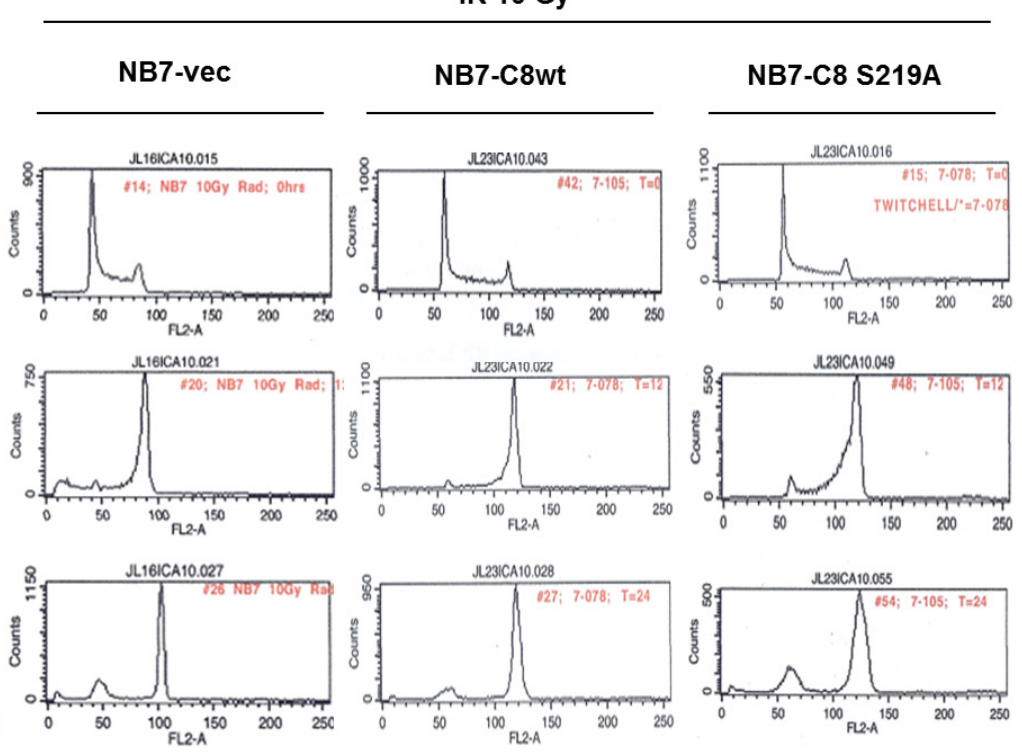

Figure A-3. Caspase-8 expression does not affect cell cycle.

Casp8 expression did not significantly affect cell cycle. NB7 cells expressing either vector (vec) or casp8wt or mutant (S219A) were synchronized using amphidicolin [5 $\mu \mathrm{g} / \mathrm{mL}]$, washed and suspended propidium iodide and analyzed by flow cytometry. Cell were analyzed every two hours for 24 hours total, shown here are just the representative 12 hour time points. 


\section{VITA}

Devin Twitchell was born in Carson City, Nevada in 1979. He graduated high school from Springville High School in 1998. He obtained his Bachelor of Science degree from Brigham Young University in 2005. In 2008, he enrolled in the Integrated Program of Biomedical Sciences doctoral program at the University of Tennessee Health Science Center. He is expected to obtain his Ph.D. degree in August 2013. 